\title{
The superconformal index and an elliptic algebra of surface defects
}

\author{
Mathew Bullimore, ${ }^{a}$ Martin Fluder, ${ }^{b}$ Lotte Hollands ${ }^{b}$ and Paul Richmond ${ }^{b}$ \\ ${ }^{a}$ Perimeter Institute for Theoretical Physics, \\ Waterloo, Ontario, N2L 2Y5, Canada \\ ${ }^{b}$ Mathematical Institute, University of Oxford, \\ Andrew Wiles Building, Radcliffe Observatory Quarter, \\ Woodstock Road, Oxford, OX2 6GG, U.K. \\ E-mail: mbullimore@perimeterinstitute.ca, fluder@maths.ox.ac.uk, \\ hollands@maths.ox.ac.uk, richmond@maths.ox.ac.uk
}

ABSTRACT: In this paper we continue the study of the superconformal index of fourdimensional $\mathcal{N}=2$ theories of class $\mathcal{S}$ in the presence of surface defects. Our main result is the construction of an algebra of difference operators, whose elements are labeled by irreducible representations of $A_{N-1}$. For the fully antisymmetric tensor representations these difference operators are the Hamiltonians of the elliptic Ruijsenaars-Schneider system. The structure constants of the algebra are elliptic generalizations of the Littlewood-Richardson coefficients. In the Macdonald limit, we identify the difference operators with local operators in the two-dimensional TQFT interpretation of the superconformal index. We also study the dimensional reduction to difference operators acting on the three-sphere partition function, where they characterize supersymmetric defects supported on a circle, and show that they are transformed to supersymmetric Wilson loops under mirror symmetry. Finally, we compare to the difference operators that create 't Hooft loops in the fourdimensional $\mathcal{N}=2^{*}$ theory on a four-sphere by embedding the three-dimensional theory as an S-duality domain wall.

Keywords: Supersymmetric gauge theory, Wilson, 't Hooft and Polyakov loops, Nonperturbative Effects, Topological Field Theories

ARXiv EPRINT: 1401.3379 


\section{Contents}

1 Introduction $\quad 2$

2 Elliptic algebra of four-dimensional surface defects $\quad 8$

2.1 The superconformal index 8

2.2 Surface defects from vortices $\quad 9$

2.3 Composition of difference operators 11

$\begin{array}{ll}2.4 \text { The algebra of surface defects } & 11\end{array}$

2.4.1 Rank $1 \quad 13$

$\begin{array}{ll}2.4 .2 \text { Higher rank } & 15\end{array}$

$\begin{array}{lll}2.5 & \text { Properties of difference operators } & 17\end{array}$

2.6 Two-dimensional worldvolume theory 18

3 Two-dimensional TQFT and Verlinde algebra 21

$\begin{array}{lll}3.1 & \text { TQFT structure of the superconformal index } & 21\end{array}$

3.1.1 Operator algebra from Macdonald polynomials 24

$\begin{array}{ll}3.2 & \text { Wilson loops in refined Chern-Simons theory }\end{array}$

4 Algebra of three-dimensional line defects $\quad 25$

4.1 From superconformal index to 3d partition function 26

$\begin{array}{lll}4.2 & \text { Wilson loops in 3d star-shaped quivers } & 28\end{array}$

$\begin{array}{lll}4.2 .1 & \mathcal{T}(\mathrm{SU}(2)) & 30\end{array}$

$\begin{array}{lll}4.2 .2 & \mathcal{T}(\mathrm{SU}(N)) & 32\end{array}$

4.3 Three-dimensional algebra 33

5 't Hooft loops in the four-dimensional $\mathcal{N}=2^{*}$ theory 34

5.1 Four-sphere partition function 34

$\begin{array}{lll}5.2 & \text { S-duality domain wall } & 35\end{array}$

$\begin{array}{lll}5.3 & \text { Supersymmetric loop operators } & 37\end{array}$

$\begin{array}{lll}5.4 & \text { Intertwining Wilson and 't Hooft loops } & 39\end{array}$

5.5 Verlinde operators in Toda CFT 40

6 Discussion $\quad 42$

A Macdonald polynomials and the refined S-matrix 45

$\begin{array}{lll}\text { A.1 Group theory } & 45\end{array}$

A.2 Schur polynomials and the modular S-matrix 45

A.3 Macdonald polynomials and the refined S-matrix 46

$\begin{array}{ll}\text { B S-duality kernel } & 48\end{array}$

B.1 Example 49 


\section{Introduction}

Surface defects are an interesting class of non-local observables in four-dimensional gauge theories [1]. In this paper, we consider surface defects in four-dimensional $\mathcal{N}=2$ superconformal field theories of class $\mathcal{S}$, which are obtained by compactifying the partially twisted six-dimensional $(2,0)$ theory on a decorated Riemann surface $C[2,3]$. The six-dimensional $(2,0)$ theory is characterized by a Lie algebra $\mathfrak{g}$ of ADE type. In this paper we focus on the case of $A_{N-1}$. In this case, the six-dimensional $(2,0)$ theory arises as the infrared limit of the worldvolume theory on a stack of $N$ coincident M5-branes. Surface defects in fourdimensional theories of class $\mathcal{S}$ can be formed from both codimension-two and codimensionfour defects in the six-dimensional parent theory. This is summarized in table 1.

Let us first discuss codimension-two defects of the $(2,0)$ theory in six-dimensions, which are labeled by embeddings $\rho: s u(2) \rightarrow \mathfrak{g}$. These defects play an important role in the construction of theories of class $\mathcal{S}$ : a codimension-two defect that is inserted at a point on the Riemann surface $C$ and spans all four space-time dimensions corresponds to a flavor puncture in the construction of $[2,3]$ - see (i) of table 1. Alternatively, wrapping the same codimension-two defect on the whole Riemann surface $C$ leads to a surface defect in the four-dimensional theory - see (ii) of table 1. This class of surface defects has been studied, for example, in $[4,5]$.

On the other hand, there are codimension-four defects in the $(2,0)$ theory in sixdimensions, which are expected to be labeled by an irreducible representation of $\mathfrak{g}$, see for example [6] and references therein. Inserting a codimension-four defect at a point on the Riemann surface $C$ engineers another class of surface defects in the four-dimensional theory - see (iii) of table 1 . In this paper, we study this second class of surface defects in four-dimensional $\mathcal{N}=2$ theories of class $\mathcal{S}$.

Important evidence for the classification of codimension-four defects in terms of irreducible representations of $\mathfrak{g}$ comes from the correspondence between four-sphere partition functions of $\mathcal{N}=2$ theories of class $\mathcal{S}$ and correlation functions in Liouville or Toda conformal field theory on $C[7,8]$. In this correspondence, flavor punctures are represented by vertex operators labeled by non-degenerate and semi-degenerate representations of the Virasoro or $W_{N}$-algebra. There are also completely degenerate representations labeled by two dominant integral weights of $\mathfrak{g}$, or equivalently, by two irreducible representations $R_{1}$ and $R_{2}$ of $\mathfrak{g}$. Correlation functions with additional insertions of completely degenerate vertex operators compute the four-sphere partition function in the presence of surface defects [9]. In particular, the labels $R_{1}$ and $R_{2}$ characterize the surface defects supported on orthogonal two-spheres.

Inspired by the connection to degenerate vertex operators and the analytic structure of Virasoro/ $W_{N}$-algebra conformal blocks, the authors of reference [10] introduced a renormalization group flow that can be used to construct the surface defects from vortex 


\begin{tabular}{|c|c|c|c|}
\hline & $X$ & $C$ & Name \\
\hline (i) & 4 & 0 & flavor puncture \\
(ii) & 2 & 2 & surface defect \\
(iii) & 2 & 0 & surface defect \\
\hline
\end{tabular}

Table 1. Summary of the defects in the six-dimensional $(2,0)$ theory on $X \times C$. $X$ is the four dimensional space-time and $C$ is a decorated Riemann surface. (i) and (ii) show configurations of codimension-two defects while (iii) shows the configuration of a codimension-four defect.

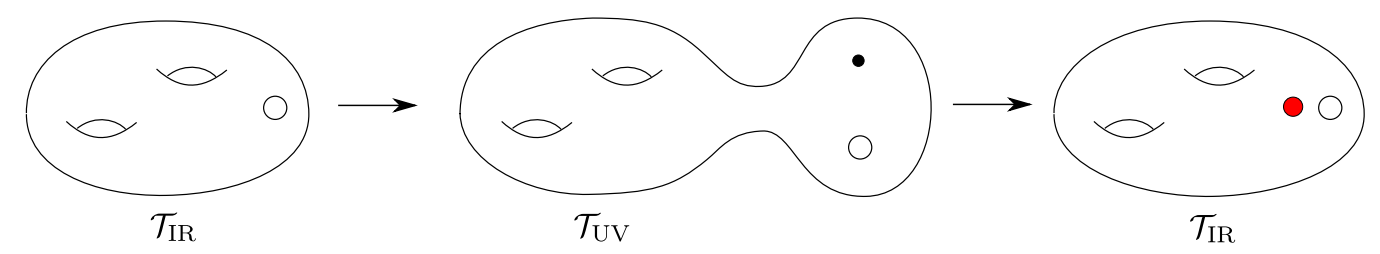

Figure 1. Schematic illustration of the renormalization group flow $\mathcal{T}_{U V} \rightarrow \mathcal{T}_{I R}$ that can be used to introduce surface defects. The white dots represent full punctures with $\mathrm{SU}(N)$ symmetry while the black dot is a simple puncture with $\mathrm{U}(1)$ symmetry. The red dot represents a codimension-four defect engineering a surface defect in four dimensions.

configurations in a larger theory. Let us consider the simplest example of this procedure illustrated in figure 1.

The starting point is a theory $\mathcal{T}_{I R}$ with a full puncture encoding an $\mathrm{SU}(N)$ flavor symmetry. We then form the larger theory $\mathcal{T}_{U V}$ by adding a simple puncture nearby with $\mathrm{U}(1)$ flavor symmetry. This corresponds to adding an additional hypermultiplet in the bifundamental of $\mathrm{SU}(N) \times \mathrm{SU}(N)$ by gauging the diagonal $\mathrm{SU}(N)$. The extra $\mathrm{U}(1)$ symmetry corresponds to the baryonic symmetry of the bifundamental hypermultiplet and the position of the simple puncture controls the gauge coupling of the gauged $\mathrm{SU}(N)$.

The theories $\mathcal{T}_{I R}$ and $\mathcal{T}_{U V}$ are connected by a renormalization group flow that is initiated by turning on a constant vacuum expectation value for the hypermultiplet scalar. By turning on a position-dependent vacuum expectation value corresponding to a half-BPS vortex configuration in $\mathcal{T}_{U V}$, the endpoint of the renormalization group flow is a surface defect in the original theory $\mathcal{T}_{I R}$. These surface defects are labeled by a pair of positive integers $\left(r_{1}, r_{2}\right)$ corresponding to the vortex numbers in orthogonal two-planes. This construction is analogous to the Toda construction of codimension-four surface operators [9]. Hence our working conjecture is that they give a representation of codimensions-four surface defects labelled by a pair of symmetric tensor representations of $\mathfrak{g}$.

A concrete prescription was given in [10] to implement this renormalization group flow at the level of the superconformal index. The superconformal index is a trace over states of a superconformal field theory in radial quantization [11]. It is a much simpler observable than the four-sphere partition function because it does not depend on the marginal couplings of the theory. For previous work on the superconformal index of theories of class $\mathcal{S}$ see [12-16]. In full generality, the $\mathcal{N}=2$ superconformal index depends on three parameters denoted by $\{p, q, t\}$ that are associated to combinations of bosonic conserved charges commuting 
with a chosen supercharge. It also depends on flavor parameters $\left\{a_{1}, \ldots, a_{N}\right\}$, such that $\prod_{j} a_{j}=1$, for each global SU $(N)$ symmetry and an additional parameter $b$ for each $\mathrm{U}(1)$ symmetry. The superconformal index is thus denoted by

$$
\mathcal{I}\left(p, q, t, a_{j}, b, \ldots\right) .
$$

The superconformal index of the theory $\mathcal{T}_{I R}$ with surface defects is obtained by computing a residue of the superconformal index of the theory $\mathcal{T}_{U V}$ in the additional fugacity $b$ associated to the additional $\mathrm{U}(1)$ symmetry. The result is a difference operator $G_{r_{1}, r_{2}}$ that acts on the superconformal index of the original theory $\mathcal{T}_{I R}$ by shifting the fugacities of the $\mathrm{SU}(N)$ flavor symmetry. Schematically, the difference operator is defined by

$$
G_{r_{1}, r_{2}} \cdot \mathcal{I}_{I R}\left(a_{j}, \ldots\right) \sim \underset{b=t^{\frac{1}{2}} p^{r_{1} / N} q^{r_{2} / N}}{\operatorname{Res}}\left[\frac{1}{b} \mathcal{I}_{U V}\left(a_{j}, b, \ldots\right)\right],
$$

where the proportionality constant is discussed in section 2 . The difference operator $G_{r_{1}, r_{2}}$ corresponds to inserting a surface defect in the original theory $\mathcal{T}_{I R}$ that is labeled by the pair $\left(r_{1}, r_{2}\right)$.

In what follows we concentrate on the case $r_{1}=0$ and simply label the difference operators by $G_{r}$, where $r \in \mathbb{Z}_{\geq 0}$. The label $r$ can be thought of as denoting a symmetric tensor representation of rank $r$. The resulting expression for $G_{r}$ is

$$
G_{r} \cdot \mathcal{I}\left(a_{j}\right)=\sum_{\sum_{k=1}^{N} m_{k}=r} \prod_{j, k=1}^{N}\left[\prod_{m=0}^{m_{k}-1} \frac{\theta\left(q^{m+m_{k}-m_{j}} t a_{j} / a_{k} ; p\right)}{\theta\left(q^{m-m_{k}} a_{k} / a_{j} ; p\right)}\right] \mathcal{I}\left(a_{j} \mapsto q^{\frac{r}{N}-m_{j}} a_{j}\right),
$$

where the theta-function $\theta(z, p)$ is defined in section 2 .

Following our arguments above, we expect that there exist difference operators $G_{R}$ corresponding to surface defects labeled by all irreducible representations $R$ of $\mathfrak{g}$. In principle, they could be constructed by starting from a theory $\mathcal{T}_{U V}$ with an additional puncture with a larger flavor symmetry. However, this would involve non-Lagrangian ingredients and, although the index can be bootstrapped as in [10], the analytic structure needed for this approach is not manifest.

Instead we follow the line of reasoning introduced in [17] and complete the algebra of difference operators. For the difference operator associated to the representation $R$ we make an ansatz

$$
G_{R} \cdot \mathcal{I}\left(a_{j}\right)=\sum_{\lambda} C_{R, \lambda}\left(p, q, t, a_{j}\right) \mathcal{I}\left(q^{-\left(\lambda, h_{j}\right)} a_{j}\right),
$$

where the sum is over the weights $\lambda$ of the representation $R,($,$) is the standard inner$ product on the Cartan subalgebra of $\mathfrak{g}$, and $h_{j}$ are the weights of the fundamental representation. This ansatz is compatible with what we already know about difference operators $G_{r}$ for symmetric tensor representations $R=(r)$.

The coefficients $C_{R, \lambda}\left(p, q, t, a_{j}\right)$ are then determined by imposing that the full set of difference operators $G_{R}$ is closed under composition

$$
G_{R_{1}} \circ G_{R_{2}}=\sum_{R_{3}} \mathcal{N}_{R_{1}, R_{2}}{ }^{R_{3}}(p, q, t) G_{R_{3}},
$$


and forms a commutative algebra. Since the symmetric tensor representations form an over-complete basis, there are many compatibility conditions for the system (1.5) to be solved consistently. It is thus non-trivial that a solution exists. Nevertheless, we can find a solution using the following method.

First, we notice that all irreducible representations in the case $\mathfrak{g}=s u(2)$ are symmetric tensor representations, so that there are no additional difference operators. Even though it is not obvious and requires numerous functional identities for theta-functions, the system (1.5) can be solved uniquely in this case. The structure coefficients $\mathcal{N}_{R_{1}, R_{2}}{ }^{R_{3}}(p, q, t)$ turn out to be an elliptic generalization of the $(q, t)$-deformed Littlewood-Richardson coefficients. In section 2.4.1 we give a recipe to obtain the elliptic coefficients $\mathcal{N}_{R_{1}, R_{2}}{ }^{R_{3}}(p, q, t)$ uniquely from the $(q, t)$-deformed ones.

If we then assume that for any rank of the gauge group the structure coefficients $\mathcal{N}_{R_{1}, R_{2}}{ }^{R_{3}}(p, q, t)$ are given by this elliptic generalization of the Littlewood-Richardson coefficients, the system (1.5) can be solved consistently and uniquely for all of the difference operators $G_{R}$. The coefficients $C_{R, \lambda}$ are in general sums of products of ratios of thetafunctions. Let us stress once more that the fact that we can find a consistent solution to the system (1.5) is highly non-trivial and involves numerous identities for theta-functions. We see this as strong evidence that a class of surface defects labeled by general irreducible representations $R$ of $\mathfrak{g}$ exists.

In particular, we find that the difference operators $G_{\left(1^{r}\right)}$ labeled by the rank $r$ antisymmetric tensor representations, can be conjugated to the Hamiltonians of the $N$-body elliptic Ruijsenaars-Schneider integrable system. This is an extension of the fact, noted in [10], that the fundamental operator in the case of $A_{1}$ can be conjugated to the Hamiltonian of the two-body elliptic Ruijsenaars-Schneider integrable system.

A microscopic definition of a large class of surface defects can be given by coupling the four-dimensional theory to two-dimensional $\mathcal{N}=(2,2)$ degrees of freedom supported on the surface $[9,18-20]$. The superconformal index in the presence of such surface defects has been constructed recently in [21]. Thus it is natural to ask whether the surface defects introduced by the operators $G_{R}$ can be understood in this approach. For the rank $r$ symmetric tensor representation, it was already noted in [21] that the two-dimensional degrees of freedom consist of an $\mathcal{N}=(2,2)$ gauge theory with gauge group $\mathrm{U}(r)$, coupled to $N$ fundamental and $N$ anti-fundamental chiral fields and an additional chiral field in the adjoint representation of $\mathrm{U}(r)$. Using the same techniques, we find that the relevant two-dimensional degrees of freedom for the rank $r$ antisymmetric tensor representation are the same as above, but without the adjoint chiral field. For other representations, it is not clear to us whether the surface defect can be constructed by coupling to an $\mathcal{N}=(2,2)$ supersymmetric gauge theory. We make a few additional remarks about this in the discussion in section 6 .

The superconformal index of $\mathcal{N}=2$ theories of class $\mathcal{S}$ has a dual description in terms of a two-dimensional topological quantum field theory on the surface $C[14,15]$. We continue in this paper by showing that the difference operators $G_{R}$ are natural objects in this two-dimensional TQFT. When we focus on the Macdonald slice $\{p=0, q, t\}$, the TQFT is given as an analytic continuation of refined Chern-Simons theory on $S^{1} \times C$ [22]. 


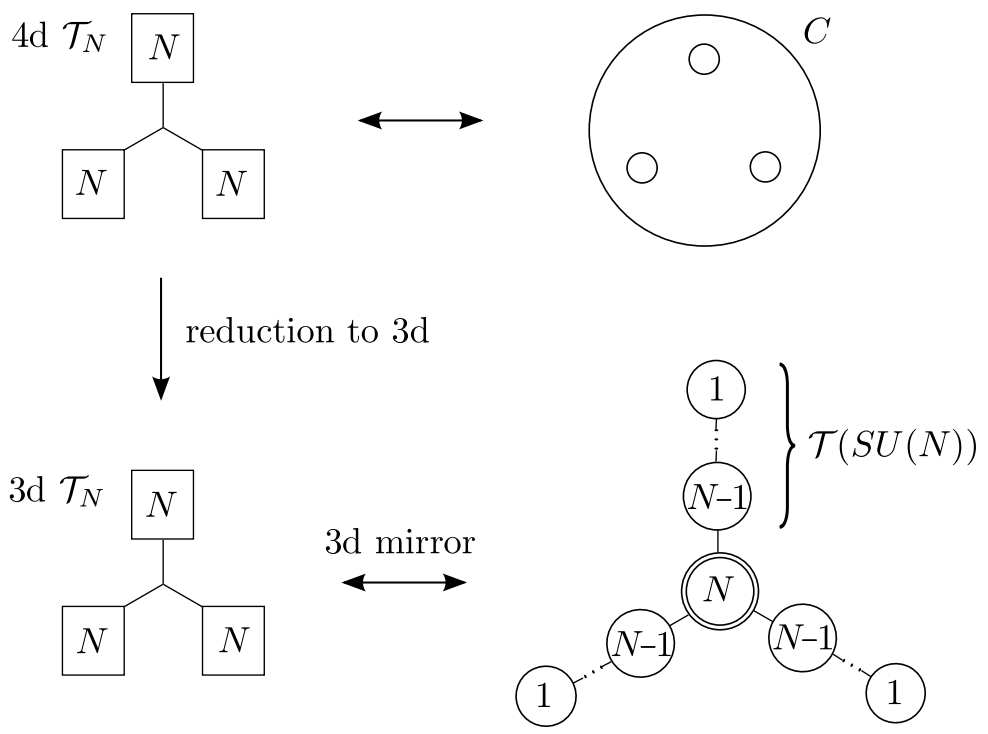

Figure 2. Sequence of dualities that maps the four-dimensional $\mathcal{T}_{N}$ theory (upper-left) to the three-dimensional star-shaped quiver theory (lower-right).

In the Macdonald limit, the operators $G_{\left(1^{r}\right)}$, labeled by antisymmetric tensor representations, can be conjugated to the so-called Macdonald operators, whose eigenfunctions are the Macdonald polynomials $P_{S}(a, q, t)$ labeled by an irreducible representation $S$. We find that the eigenvalue of a general, conjugated, difference operator $G_{R}^{c}$ in the Macdonald limit is given by

$$
G_{R}^{c} \cdot P_{S}\left(a_{j}, q, t\right)=\frac{S_{R, S}}{S_{0, S}} P_{S}\left(a_{j}, q, t\right),
$$

where $S_{R, S}$ is an analytic continuation of the modular S-matrix of refined Chern-Simons theory, which depends on $q$ and $t$. A consequence is that the surface defect introduced by the operator $G_{R}^{c}$ is equivalent to a Wilson loop wrapping around the $S^{1}$ of the threemanifold $S^{1} \times C$.

In the Macdonald limit, the structure constants $\mathcal{N}_{R_{1}, R_{2}}{ }^{R_{3}}(q, t)$ become the $(q, t)$ deformed Littlewood-Richardson coefficients and the algebra of difference operators $G_{R}$ is identified with the Verlinde algebra. We expect that this Verlinde algebra has a natural interpretation in the (analytically continued) chiral boundary theory on the two-torus boundary near a puncture of $C$.

We find further confirmation of the physical relevance of the difference operators $G_{R}$ by reducing the superconformal index to the three-sphere partition function, following [2325]. In particular, we consider the dimensional reduction of the four-dimensional $\mathcal{T}_{N}$ theory, which is obtained by compactifying the six-dimensional $(2,0)$ theory on a three-punctured sphere with three full punctures. The dimensionally reduced $\mathcal{T}_{N}$ theory has a Lagrangian mirror description as a star-shaped quiver theory [26]. This is illustrated in figure 2. In particular, each full puncture of the three-punctured sphere is represented by a threedimensional linear quiver theory called $\mathcal{T}(\mathrm{SU}(N))$. 
It is expected that the surface defects introduced by the dimensional reduction of the operators $G_{R}$ correspond to supersymmetric Wilson loops in the representation $R$ for the central node of the star-shaped quiver. This is in fact equivalent to the statement that the partition function of the $\mathcal{T}(\mathrm{SU}(N))$ theory is an eigenfunction of the dimensionally reduced operators $G_{R}^{(3 \mathrm{~d})}$. The partition function $\mathcal{Z}(x, y)$ of the $\mathcal{T}(\mathrm{SU}(N))$ theory depends on two mass parameters $x$ and $y$ associated to the Higgs branch and the Coulomb branch respectively, and is symmetric under $x \leftrightarrow y$. For the case of a round four-sphere, we show indeed that

$$
G_{\left(1^{r}\right)}^{(3 \mathrm{~d})}(y) \cdot \mathcal{Z}(x, y)=W_{\left(1^{r}\right)}(x) \mathcal{Z}(x, y)
$$

where $W_{\left(1^{r}\right)}(x)$ is a supersymmetric Wilson loop in the $r$-th antisymmetric tensor representation.

For other (non-minuscule) representations we find that this is not quite correct. In particular, the Wilson loops obey the algebra

$$
W_{R_{1}} \cdot W_{R_{2}}=\sum_{R_{3}} N_{R_{1}, R_{2}}^{R_{3}} W_{R_{3}},
$$

where $N_{R_{1}, R_{2}}{ }^{R_{3}}$ are the ordinary Littlewood-Richardson coefficients, whereas the algebra of the three-dimensional operators $G_{R}^{(3 \mathrm{~d})}$ is not of this form. Instead, we find that when the representation $R$ is non-minuscule, the dimensionally reduced operators $G_{R}^{(3 \mathrm{~d})}$ are linear combinations of operators $\tilde{G}_{S}^{(3 \mathrm{~d})}$, with $|S| \leq|R|$, that are dual to Wilson loop operators. ${ }^{1}$ This gives a simple invertible linear transformation on the algebra of difference operators.

Finally, by embedding the three-dimensional $\mathcal{T}(\mathrm{SU}(N))$ theory as an S-duality domain wall in the four-dimensional $\mathcal{N}=2^{*}$ theory, we interpret the dimensionally reduced difference operators $G_{R}^{(3 \mathrm{~d})}$ as operators that introduce 't Hooft defects, labeled by irreducible representations $R$, into the four-sphere partition function of the $\mathcal{N}=2^{*}$ theory. Again, when the representation $R$ is an antisymmetric tensor representation, we find perfect agreement with both localization [27] and (in the case of the fundamental representation) computations of Verlinde operators in Liouville/Toda conformal field theory [9, 28-30], while for other representations we once more find an invertible linear transformation on the algebra of operators.

The outline of this paper is as follows. In section 2 we construct the difference operators $G_{R}$ by completing the algebra generated by the difference operators $G_{r}$, which are labeled by symmetric tensor representations, and we interpret the operators $G_{R}$ as computing the $\mathcal{N}=2$ superconformal index in the presence of surface defects. In section 3 we interpret the difference operators $G_{R}$ in the limit $p=0$ as Wilson loops wrapping the $S^{1}$ in an analytic continuation of refined Chern-Simons theory on $S^{1} \times C$. In section 4 we reduce the difference operators $G_{R}$ to three dimensions, and interpret them as operators that describe line defects when added to the three-sphere partition function. In section 5 we relate the dimensionally reduced operators $G_{R}^{(3 \mathrm{~d})}$ to operators that introduce 't Hooft loops into the four-sphere

\footnotetext{
${ }^{1}$ We defined the partial ordering of representation by $\left|R_{1}\right|<\left|R_{2}\right|$ iff the dimension of the representation $R_{1}$ is less than the dimension of $R_{2}$.
} 
partition function of the four-dimensional $\mathcal{N}=2^{*}$ theory. We finish in section 6 with a discussion of our findings. Some longer calculations are presented in appendices A, B and C.

\section{Elliptic algebra of four-dimensional surface defects}

\subsection{The superconformal index}

The superconformal index is a trace over the states of a superconformal field theory in radial quantization, or equivalently, a twisted partition function on $S^{1} \times S^{3}$. The most general superconformal index of four-dimensional $\mathcal{N}=2$ theories is

$$
\mathcal{I}=\operatorname{Tr}(-1)^{F} p^{j_{z}-r} q^{j_{w}-R} t^{r+R} \prod_{j} a_{j}^{f_{j}},
$$

where the trace is taken over states of the theory in radial quantization annihilated by a single supercharge $\tilde{Q}_{1, \dot{-}}$. Here, we are parametrizing $S^{3}$ by two complex coordinates $(z, w)$ obeying $|z|^{2}+|w|^{2}=1$, and the generators $j_{z}$ and $j_{w}$ are rotations in the orthogonal $z$ and $w$-planes respectively. The symbol $r$ denotes the generator of the superconformal $\mathrm{U}(1)_{r}$ and $R$ the generator of the Cartan subalgebra of $\mathrm{SU}(2)_{R}$. The $f_{j}$ are generators of the Cartan subalgebra of the flavor symmetry group.

The combinations of generators appearing in the powers of $\left(p, q, t, a_{j}\right)$ in equation (2.1) are those combinations that commute with the supercharge $\tilde{Q}_{1,-}$. The letters $p, q, t$ and $a_{i}$ are fugacities for these symmetries and obey

$$
|p|,|q|,|t|,|p q / t|<1, \quad\left|a_{j}\right|=1,
$$

which ensure that the index is well-defined.

If there exists a weakly coupled Lagrangian, the superconformal index can be computed from single-letter indices by the plethystic exponential. The basic ingredients are the single letter indices of a half-hypermultiplet and vectormultiplet,

$$
\begin{aligned}
& i_{H}=\frac{\sqrt{t}-\frac{p q}{\sqrt{t}}}{(1-p)(1-q)}, \\
& i_{V}=-\frac{p}{1-p}-\frac{q}{1-q}+\frac{\frac{p q}{t}-t}{(1-p)(1-q)} .
\end{aligned}
$$

For example, the superconformal index of a free hypermultiplet in the bifundamental representation of $\mathrm{SU}(N) \times \mathrm{SU}(N)$ is

$$
\begin{aligned}
\mathcal{I}\left(a_{j}, b_{j}, c\right) & =\operatorname{PE}\left[i_{H} \sum_{i, j=1}^{N}\left(a_{i} b_{j} c+\frac{1}{a_{i} b_{j} c}\right)\right] \\
& =\prod_{i, j=1}^{N} \Gamma\left(\sqrt{t}\left(a_{i} b_{j} c\right)^{ \pm} ; p, q\right),
\end{aligned}
$$

where PE stands for the plethystic exponential. The parameters $\left\{a_{i}\right\}$ and $\left\{b_{j}\right\}$ are fugacities for the $\mathrm{SU}(N) \times \mathrm{SU}(N)$ symmetry and $c$ is the fugacity for the overall $\mathrm{U}(1)$ symmetry. The 
elliptic gamma function $\Gamma(z ; p, q)$ is defined as

$$
\Gamma(z ; p, q)=\prod_{i, j=0}^{\infty} \frac{\left(1-z^{-1} p^{i+1} q^{j+1}\right)}{\left(1-z p^{i} q^{j}\right)} .
$$

An important operation on the superconformal index is that of gauging a global symmetry. Given the superconformal index $\mathcal{I}(a)$ of a theory with $\mathrm{SU}(N)$ flavor symmetry, the superconformal index of the theory where this symmetry has been gauged is

$$
\oint \Delta(a) \mathcal{I}_{V}(a) \mathcal{I}(a)
$$

where

$$
\mathcal{I}_{V}(a)=\operatorname{PE}\left[i_{V}\left(\sum_{i, j=1}^{N} \frac{a_{i}}{a_{j}}-1\right)\right]
$$

is the superconformal index of an $\mathrm{SU}(N)$ vectormultiplet and

$$
\Delta(a)=\left[\prod_{j=1}^{N-1} \frac{d a_{j}}{2 \pi i a_{j}}\right] \frac{1}{N !} \prod_{i \neq j}^{N}\left(1-\frac{a_{i}}{a_{j}}\right)
$$

is the Haar measure on the maximal torus of $\mathrm{SU}(N)$.

\subsection{Surface defects from vortices}

In this section, we review the construction of the superconformal index in the presence of a certain class of surface defects, which arise as the infinite tension limit of background vortex configurations [10]. They are labeled by a nonnegative integer $r$, the vortex number, which may be interpreted as the magnetic flux through the vortex core.

The starting point is any superconformal field theory $\mathcal{T}_{I R}$ with a global flavor symmetry $\mathrm{SU}(N)$. By gauging this flavor symmetry, the theory may be coupled to a hypermultiplet in the bifundamental representation of $\mathrm{SU}(N) \times \mathrm{SU}(N)$. The resulting superconformal field theory $\mathcal{T}_{U V}$ has an additional baryonic $\mathrm{U}(1)$ symmetry acting on the bifundamental hypermultiplet.

The two theories $\mathcal{T}_{I R}$ and $\mathcal{T}_{U V}$ are related by a renormalization flow initiated by turning on a Higgs branch vacuum expectation value for the bifundamental scalar field $Q$. When this expectation value is a constant, the RG flow brings us back to the theory $\mathcal{T}_{I R}$. When the expectation value is taken to be coordinate-dependent, the theory $\mathcal{T}_{I R}$ is modified along a surface and in the low energy limit we recover the theory $\mathcal{T}_{I R}$ in the presence of a surface defect.

More precisely, we can introduce a vacuum expectation value for the baryon operator $B=\operatorname{det} Q$ of the form

$$
B(z)=\prod_{i=1}^{r}\left(z-z_{i}\right),
$$

where $z$ is a complex coordinate in a two-plane, the degree $r$ corresponds to the vortex number, and the parameters $z_{i}$ are the positions of the vortex strings. Taking the $z_{i}=0$, 


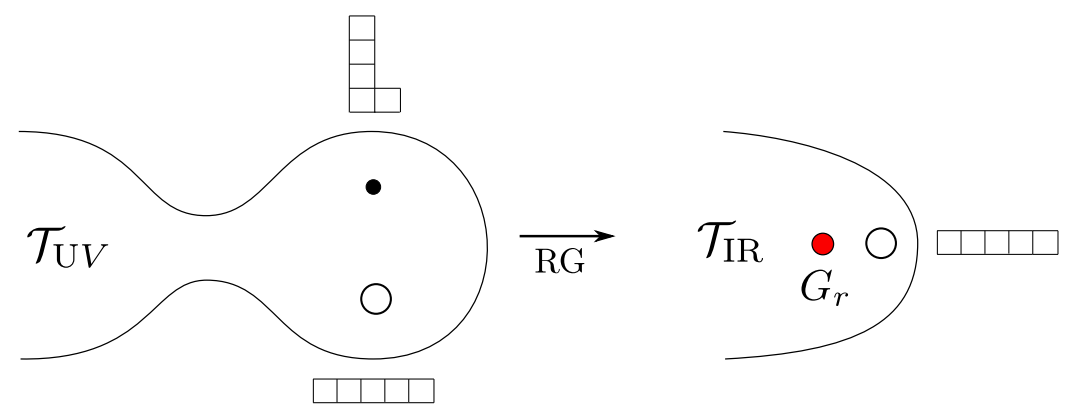

Figure 3. The left picture illustrates the Riemann surface $C$ corresponding to a theory $\mathcal{T}_{U V}$, which is obtained by coupling the theory $\mathcal{T}_{I R}$ to a bifundamental field. An RG flow, that is initiated by turning on a Higgs vev for the bifundamental scalar, relates the theory $\mathcal{T}_{U V}$ to the original theory $\mathcal{T}_{I R}$ with a surface defect $G_{r}$. This is illustrated on the right.

we have $r$ coincident vortices. This construction then leads to surface defects labeled by $r \in \mathbb{Z}_{\geq 0}$. For $\mathcal{N}=2$ superconformal field theories of class $\mathcal{S}$, this construction has an elegant interpretation in terms of the curve $C$ - see figure 3 .

This field theoretic construction of surface defects can be implemented concretely in the superconformal index for surface defects supported on the $S^{1} \times S^{1}$ defined by the locus $\{z=$ $0\}$. Denoting the superconformal index of $\mathcal{T}_{I R}$ by $\mathcal{I}_{I R}\left(a_{j}, \ldots\right)$, then the superconformal index of $\mathcal{T}_{U V}$ is

$$
\mathcal{I}_{U V}\left(b_{j}, c, \ldots\right)=\oint \Delta\left(a_{i}\right) \mathcal{I}_{V}\left(a_{i}\right) \mathcal{I}_{H}\left(a_{i}, b_{j}, c\right) \mathcal{I}_{\mathrm{IR}}\left(a_{i}^{-1}, \ldots\right) .
$$

This has simple poles that originate from simple poles in the integrand pinching the contour. We consider the simple poles of the integrand coming from the bifundamental hypermultiplet index at

$$
a_{i}=t^{\frac{1}{2}} q^{m_{i}} \frac{1}{b_{\sigma(i)} c}
$$

where $\sigma$ is a permutation of $\{1, \ldots, N\}$ and $\sum_{i} m_{i}=r$ where $r \in \mathbb{Z}_{\geq 0}$. They correspond to the chiral ring generated by derivatives of components of the bifundamental scalar field, $\left(\partial_{w}\right)^{m_{i}} Q_{i}^{\sigma(i)}$. For each permutation $\sigma$, these poles pinch the contour when

$$
c=t^{\frac{1}{2}} q^{\frac{r}{N}}
$$

leading to a simple pole in the integral at this point. This pole then corresponds to the chiral ring generated by derivatives of the baryon operator $\left(\partial_{w}\right)^{r} B$ where $B=\operatorname{det} Q$, which is charged only under the $\mathrm{U}(1)$. The residue at this pole corresponds to the index of $\mathcal{T}_{I R}$ in the presence of a surface defect obtained by giving an expectation value $B=z^{r}$ to the baryon operator of $\mathcal{T}_{U V}$ and flowing to the IR.

As demonstrated in [10], the residue takes the form of a difference operator $G_{r}$ acting on the superconformal index of $\mathcal{T}_{I R}$. There is one term in the operator for each distinct set of integers $\left\{m_{1}, \ldots, m_{N}\right\}$ such that $\sum_{i} m_{i}=r$. The precise prescription defining the difference operator is

$$
G_{r} \cdot \mathcal{I}_{I R}\left(b_{i}, \ldots\right)=N \mathcal{I}_{V}\left(b_{i}\right) \operatorname{Res}_{c=t^{\frac{1}{2}} q^{\frac{r}{N}}}\left[\frac{1}{c} \mathcal{I}_{\mathrm{UV}}\left(c, b_{i}, \ldots\right)\right]
$$


The result of the computation is

$$
G_{r} \cdot \mathcal{I}\left(b_{i}\right)=\sum_{\sum_{j=1}^{N} m_{j}=r} \prod_{i, j=1}^{N}\left[\prod_{m=0}^{m_{j}-1} \frac{\theta\left(q^{m+m_{j}-m_{i}} t b_{i} / b_{j} ; p\right)}{\theta\left(q^{m-m_{j}} b_{j} / b_{i} ; p\right)}\right] \mathcal{I}\left(b_{i} \mapsto q^{\frac{r}{N}-m_{i}} b_{i}\right)
$$

where the theta-function is defined as

$$
\theta(z ; p)=\prod_{i=0}^{\infty}\left(1-z p^{i}\right)\left(1-\frac{p^{i+1}}{z}\right)
$$

The difference operators $G_{r}$ constructed by this method are formally self-adjoint with respect to the measure $\Delta(a) \mathcal{I}_{V}(a)$ used for gauging. They are labeled by a nonnegative integer $r \in \mathbb{Z}_{\geq 0}$. Furthermore each term in the operator can be identified with a weight of the $r$-th symmetric tensor representation of $s u(N)$. In particular, the numbers $\left\{m_{1}, m_{2}, \ldots, m_{N}\right\}$ denote the number of times the integers $\{1, \ldots, N\}$ appear in the corresponding Young tableau. Based on this observation, we associate these operators to surface defects labeled by the symmetric tensor representations of $s u(N)$.

It is, however, expected that there exist surface defects labeled by arbitrary irreducible representations of $s u(N)$. The necessity of such defects becomes apparent when the difference operators are composed.

\subsection{Composition of difference operators}

Let us now consider the composition of two difference operators, $G_{r_{1}} \circ G_{r_{2}}$. This can be given a physical interpretation by coupling the theory $\mathcal{T}_{I R}$ to a single hypermultiplet $Q_{1}$ in the bifundamental representation of $\mathrm{SU}(N) \times \mathrm{SU}(N)$ and then to another bifundamental hypermultiplet $Q_{2}$. The resulting theory $\mathcal{T}_{U V}^{\prime}$ is illustrated in figure 4 . It has two additional flavor symmetries $\mathrm{U}(1)_{1}$ and $\mathrm{U}(1)_{2}$ that act on the two bifundamental hypermultiplets $Q_{1}$ and $Q_{2}$ respectively.

The original theory $\mathcal{T}_{I R}$ is reached by turning on constant vacuum expectation values for both baryon operators $B_{1}=\operatorname{det} Q_{1}$ and $B_{2}=\operatorname{det} Q_{2}$ charged under the additional flavor symmetries $\mathrm{U}(1)_{1}$ and $\mathrm{U}(1)_{2}$. In the superconformal index, this corresponds to the residues of $\mathcal{I}_{U V}$ at the simple poles $c_{1}=t^{1 / 2}$ and $c_{2}=t^{1 / 2}$ in the fugacities associated to $\mathrm{U}(1)_{f, 1}$ and $\mathrm{U}(1)_{f, 2}$ respectively. Turning on position dependent vacuum expectation values $B_{1}=z^{r_{1}}$ and $B_{2}=z^{r_{2}}$ corresponds to computing the residues at simple poles $c_{1}=t^{1 / 2} q^{r_{1}}$ and $c_{2}=t^{1 / 2} q^{r_{2}}$. The order in which the residues are computed is irrelevant and the result

$$
G_{r_{1}} \cdot\left(G_{r_{2}} \cdot \mathcal{I}_{I R}\right)=G_{r_{2}} \cdot\left(G_{r_{1}} \cdot \mathcal{I}_{I R}\right)
$$

defines the (commutative) composition $G_{r_{1}} \circ G_{r_{2}}$. This construction again has an interpretation in terms of the curve $C$ for theories of class $\mathcal{S}$, shown in figure 4 .

\subsection{The algebra of surface defects}

The operators $G_{r}$ constructed above do not form a closed algebra under composition and addition. More precisely, except for $s u(2)$, the composition $G_{r_{1}} \circ G_{r_{2}}$ cannot be decomposed 

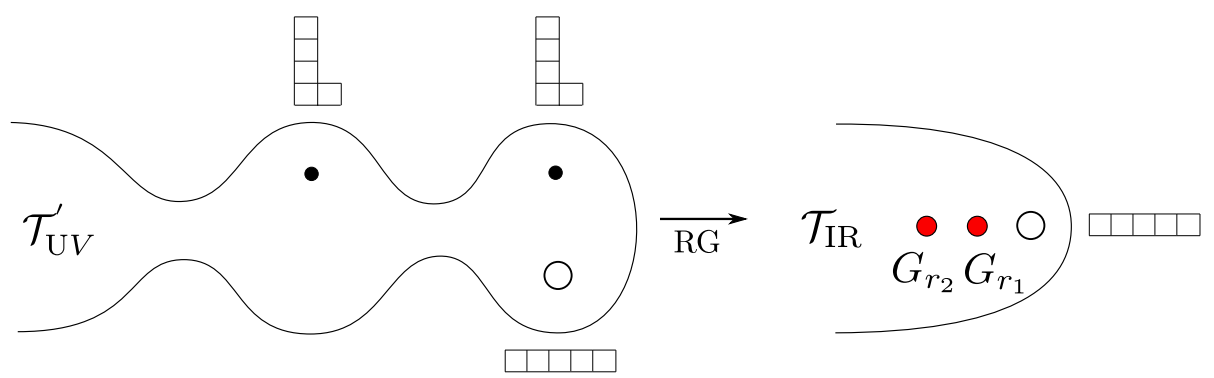

Figure 4. The left picture illustrates the Riemann surface $C$ corresponding to the theory $\mathcal{T}_{U V}^{\prime}$, which is obtained by coupling the theory $\mathcal{T}_{I R}$ to two bifundamental fields. An RG flow, that is initiated by turning on Higgs vevs for both bifundamental scalars, relates the theory $\mathcal{T}_{\mathrm{UV}}^{\prime}$ to the original theory $\mathcal{T}_{\text {IR }}$ with two surface defects $G_{r_{1}}$ and $G_{r_{2}}$. This is illustrated on the right.

as a sum of other operators $G_{r_{3}}$ with coefficients that are independent of the flavor fugacities $\left\{a_{j}\right\}$ acted on by the operators.

In order to close the algebra, we need to enlarge the set of difference operators $G_{r}$. Having identified the label $r$ with the $r$-fold symmetric tensor representation of $s u(N)$, it is natural to introduce operators $G_{R}$ for any irreducible representation $R$ of $s u(N)$ and to force them to obey the algebra

$$
G_{R_{1}} \circ G_{R_{2}}=\sum_{R_{3}} \mathcal{N}_{R_{1}, R_{2}}^{R_{3}} G_{R_{3}}
$$

where the coefficient $\mathcal{N}_{R_{1}, R_{2}}{ }^{R_{3}}$ is non-zero only when the representation $R_{3}$ appears in the direct sum decomposition of the tensor product $R_{1} \otimes R_{2}$. Indeed, it turns out that this determines the operators $G_{R}$ and the algebra coefficients $\mathcal{N}_{R_{1}, R_{2}}{ }^{R_{3}}$ essentially uniquely, in a sense we explain in detail below. The closure of the algebra is a highly non-trivial statement, however, depending on intricate theta-function identities.

Let us explain the procedure is some more detail. For each irreducible representation $R$ of $s u(N)$, we make an ansatz for the operator $G_{R}$. The ansatz is a sum over the weights $\lambda$ of the representation $R$,

$$
G_{R} \cdot \mathcal{I}\left(a_{i}\right)=\sum_{\lambda} C_{R, \lambda}\left(p, q, t, a_{j}\right) \mathcal{I}\left(q^{-\left(\lambda, h_{i}\right)} a_{i}\right)
$$

with some unknown functions $C_{R, \lambda}\left(p, q, t, a_{j}\right)$. Here, the bracket (, ) denotes the standard inner product on the Cartan subalgebra normalized so that $\left(e_{i}, e_{i}\right)=2$ for all simple roots. Furthermore, $h_{i}$ are the weights of the fundamental representation. They obey $\left(h_{i}, h_{j}\right)=\delta_{i, j}-1 / N$.

The weights of an irreducible representation $R$ of $s u(N)$ can be represented by semistandard Young tableaux, that are obtained by placing a number $1, \ldots, N$ in each box of the Young diagram (as we review in appendix A). Each weight can be written as a sum

$$
\lambda=\sum_{j=1}^{N} m_{j} h_{j},
$$


where $m_{j}$ are the filling numbers of the corresponding semi-standard Young tableau. In particular, the weights of the $r$-th symmetric tensor representation are given by

$$
\lambda=\sum_{j=1}^{N} m_{j} h_{j}
$$

where the numbers $m_{i}$ are such that $\sum_{j} m_{j}=r$. Since $\left(\lambda, h_{i}\right)=m_{i}-\frac{r}{N}$, the chosen ansatz is compatible with the symmetric tensor operators $G_{r}$ that we already know.

Now we substitute the coefficients $C_{R, \lambda}\left(p, q, t, a_{j}\right)$ for the symmetric tensor operators, as well as our ansatz for the remaining representations, into the algebra relations

$$
G_{R_{1}} \circ G_{R_{2}}=\sum_{R_{3}} \mathcal{N}_{R_{1}, R_{2}}{ }^{R_{3}} G_{R_{3}} .
$$

We first solve these relations for the $s u(2)$ coefficients $\mathcal{N}_{r_{1}, r_{2}}{ }^{r_{3}}(p, q, t)$, and propose a generalization for the $s u(N)$ coefficients $\mathcal{N}_{R_{1}, R_{2}}{ }^{R_{3}}(p, q, t)$. Then we find that the remaining coefficients $C_{R, \lambda}\left(p, q, t, a_{j}\right)$ are determined uniquely. The fact that this procedure works requires intricate theta-function identities, providing a strong self-consistency check of our ansatz.

As a preliminary step, we introduce a small normalization of the operators $G_{r}$ labeled by $r$-th symmetric tensor representations. We redefine the operators by multiplying them by the factor

$$
\mathcal{N}_{r}=t^{-r(N-1) / 2} \prod_{i=0}^{r-1} \frac{\theta\left(q^{-1-i}, p\right)}{\theta\left(t q^{i}, p\right)} .
$$

The purpose of the normalization is to render the leading algebra coefficient equal to one. In the Schur limit $\{p, q, t\} \rightarrow\{p, q, q\}$ this normalization factor reduces to $\mathcal{N}_{r} \rightarrow$ $(-1)^{r} q^{-\frac{1}{2} r(r+N)}$, in agreement with the normalization factor in [17].

\subsubsection{Rank 1}

A good starting point is $s u(2)$, since its irreducible representations are exhausted by $r$-fold symmetric products of the fundamental representation. Thus, the algebra of difference operators should close without introducing any new operators. In particular, we expect that the product $G_{r_{1}} \circ G_{r_{2}}$ can be decomposed according to the tensor product of the corresponding irreducible representations

$$
G_{r_{1}} \circ G_{r_{2}}=\sum_{r=\left|r_{1}-r_{2}\right|}^{r_{1}+r_{2}} \mathcal{N}_{r_{1}, r_{2}}{ }^{r_{3}} G_{r_{3}},
$$

where we can compute the OPE coefficients $\mathcal{N}_{r_{1}, r_{2}}{ }^{r_{3}}(p, q, t)$. Consistency of this structure demands that the coefficients $\mathcal{N}_{r_{1}, r_{2}}{ }^{r_{3}}$ constructed in this way are independent of the fugacity parameter $a$.

For simplicity, let us first consider the Macdonald limit $p \rightarrow 0$. In this limit, the ratios of theta-functions in the operators are replaced by rational functions of the remaining variables $q$ and $t$. The operators $G_{r}$ become

$$
G_{r} \cdot \mathcal{I}\left(a_{i}\right)=\mathcal{N}_{r} \sum_{m_{1}+m_{2}=r} \prod_{i, j=1}^{2}\left[\prod_{m=0}^{m_{j}-1} \frac{\left(1-q^{m+m_{j}-m_{i}} \frac{t a_{i}}{a_{j}}\right)}{\left(1-q^{m-m_{j}} \frac{a_{j}}{a_{i}}\right)}\right] \mathcal{I}\left(a_{i} \mapsto q^{\frac{r}{N}-m_{i}} a_{i}\right),
$$


where $a_{1}=a$ and $a_{2}=a^{-1}$.

When composing any two such rational operators $G_{r_{1}}$ and $G_{r_{2}}$, we indeed find that the product $G_{r_{1}} \circ G_{r_{2}}$ decomposes according to the tensor product of the corresponding irreducible representations, in such a way that the structure constants $\mathcal{N}_{r_{1}, r_{2}}{ }^{r_{3}}(q, t)$ are rational functions of $q$ and $t$.

As mentioned above, we have normalized the difference operators such that the structure constant for the leading OPE coefficient $\mathcal{N}_{r_{1}, r_{2}}{ }^{r_{1}+r_{2}}=1$. The remaining structure constants can be computed straightforwardly in each case. For example, $G_{1} \circ G_{1}=$ $G_{2}+\mathcal{N}_{1,1}^{0} G_{0}$, where

$$
\mathcal{N}_{1,1}^{0}(q, t)=\frac{(1+t)(1-q)}{(1-q t)} .
$$

This is a particular case of the more general decomposition

$$
G_{1} \circ G_{r}=G_{r+1}+\mathcal{N}_{1, r}^{r-1} G_{r-1}
$$

where

$$
\mathcal{N}_{1, r}^{r-1}(q, t)=\frac{\left(1-t^{2} q^{r-1}\right)\left(1-q^{r}\right)}{\left(1-t q^{r-1}\right)\left(1-t q^{r}\right)} .
$$

Similar formulae can be derived for any other example.

Remarkably, we observe that the structure constants $\mathcal{N}_{r_{1}, r_{2}}{ }^{r}(q, t)$ are equal to the $(q, t)$ deformed Littlewood-Richardson coefficients. In other words, the operators $G_{r}$ in the limit $p \rightarrow 0$ obey the same algebra as the Macdonald polynomials $P_{r}(a, q, t)$ for $s u(2)$. (We refer to appendix A for more details regarding Macdonald polynomials and $(q, t)$-deformed Littlewood-Richardson coefficients.)

It turns out that the structure constants of the general elliptic operator algebra can be obtained in a canonical way by "lifting" the structure constants $\mathcal{N}_{r_{1}, r_{2}}{ }^{r_{3}}(q, t)$ of the Macdonald algebra. This works as follows. First we express the $(q, t)$-deformed LittlewoodRichardson coefficients as rational functions consisting of factors of the form $(1-x)$, where $x$ is a monomial of the form $q^{\alpha} t^{\beta}$. Then we "lift" each factor to an elliptic function $\theta(x, p)$ whose second argument is the additional parameter $p$. The original coefficients are obtained in the limit $p \rightarrow 0$.

Note that even though there are ambiguities in writing the $(q, t)$-deformed LittlewoodRichardson coefficients as rational functions of the form $(1-x)$, such as for example in

$$
\mathcal{N}_{1,1}^{0}(q, t)=\frac{\left(1-t^{2}\right)(1-q)}{(1-t)(1-q t)}=\frac{\left(1-\frac{1}{t^{2}}\right)\left(1-\frac{1}{q}\right)}{\left(1-\frac{1}{t}\right)\left(1-\frac{1}{q t}\right)},
$$

the elliptic lift

$$
\mathcal{N}_{1,1}^{0}(p, q, t)=\frac{\theta\left(t^{2}, p\right) \theta(q, p)}{\theta(t, p) \theta(q t, p)} .
$$

is uniquely defined because of the theta-function identity

$$
\theta\left(z^{-1} ; p\right)=-\frac{1}{z} \theta(z ; p)
$$


Verifying the composition rules for the elliptic difference operators $G_{r}$ now requires numerous theta-function identities. For instance, checking that $G_{1} \circ G_{1}=G_{2}+\mathcal{N}_{1,1}{ }^{0}(p, q, t) G_{0}$ requires

$$
\begin{aligned}
\frac{\theta\left(t^{2}, p\right) \theta(q, p)}{\theta(t, p) \theta(q t, p)}= & +\frac{\theta\left(q^{-2}, p\right) \theta\left(t^{-1}, p\right) \theta\left(t a^{-2}, p\right) \theta\left(t a^{2}, p\right)}{\theta\left(q^{-1}, p\right) \theta\left(q^{-1} a^{-2}, p\right) \theta\left(q^{-1} a^{2}, p\right) \theta(q t, p)} \\
& -\frac{\theta\left(t^{-1}, p\right) \theta\left(t a^{-2}, p\right) \theta\left(t q^{-1} a^{2}, p\right)}{\theta\left(a^{-2}, p\right) \theta(t, p) \theta\left(q^{-1} a^{2}, p\right)} \\
& -\frac{\theta\left(t^{-1}, p\right) \theta\left(t a^{2}, p\right) \theta\left(t q^{-1} a^{-2}, p\right)}{\theta\left(a^{2}, p\right) \theta(t, p) \theta\left(q^{-1} a^{-2}, p\right)},
\end{aligned}
$$

which can be checked for instance by expanding around $p=0$.

Similarly, when composing the fundamental operator $G_{1}$ with the operator $G_{r}$ for any other irreducible representation of $s u(2)$, we find that another elliptic theta-function identity brings the non-trivial structure constant into the form

$$
\mathcal{N}_{1, r}{ }^{r-1}(p, q, t)=\frac{\theta\left(t^{2} q^{r-1}, p\right) \theta\left(q^{r}, p\right)}{\theta\left(t q^{r-1}, p\right) \theta\left(t q^{r}, p\right)} .
$$

In fact, for any other check we did, we find that the structure constants $\mathcal{N}_{r_{1}, r_{2}}{ }^{r_{3}}$ are independent of the fugacity parameter $a$ and can be expressed as ratios of theta-functions. Even better, we find that they are elliptic (lifts of $(q, t)$-deformed) Littlewood-Richardson coefficients, in the sense explained above.

The elliptic operators $G_{r}$ thus obey an elliptic version of the Macdonald polynomial algebra. In particular, this provides evidence for the conjecture that the surface defects labeled by $r \in \mathbb{Z}_{\geq 0}$ are to be identified with irreducible representations of $s u(2)$.

\subsubsection{Higher rank}

For $\operatorname{su}(N)$, with $N>2$, the algebra of the difference operators $G_{r}$ is not closed. We introduce a new set of operators $G_{R}$ labeled by irreducible representations of $s u(N)$, and identify the difference operators $G_{r}$ with the operators $G_{(r)}$ labeled by the rank $r$ symmetric tensor representation. ${ }^{2}$ We systematically find expressions for the novel operators by imposing the algebra

$$
G_{R_{1}} \circ G_{R_{2}}=\sum_{R_{3}} \mathcal{N}_{R_{1}, R_{2}}^{R_{3}} G_{R_{3}}
$$

where we assume that the coefficients $\mathcal{N}_{R_{1}, R_{2}}{ }^{R_{3}}(p, q, t)$ are given by the elliptic (lifts of $(q, t)$ refined) Littlewood-Richardson coefficients, which can be found uniquely for any triple of representations $R_{1}, R_{2}$ and $R_{3}$.

In the rank 2 and 3 cases, we have explicitly computed a large set of elliptic difference operators $G_{R}$, and performed ample consistency checks amongst them. These computations reveal several structures amongst the difference operators, and we are to make some proposals for general $N$. Let us give a few examples here.

\footnotetext{
${ }^{2}$ We label by $\left(\ell_{1}, \ldots, \ell_{N-1}\right)$ the representation associated to the Young diagram whose $j$-th row has length $\ell_{j}$.
} 
First, consider the composition of two operators each labeled by the fundamental representation, $G_{(1)} \circ G_{(1)}$. This representation $(1) \otimes(1)$ decomposes into the symmetric tensor (2) and the antisymmetric tensor $(1,1)$ representations. The coefficient of the operator $G_{(2)}$ labeled by the symmetric tensor representation is one, following from our choice of normalization. Choose the coefficient

$$
\mathcal{N}_{(1),(1)}^{(1,1)}(p, q, t)=\frac{\theta(q, p) \theta\left(t^{2}, p\right)}{\theta(t, p) \theta(q t, p)}
$$

to be the uplift of the corresponding $(q, t)$-deformed Littlewood-Richardson coefficient. The difference operator $G_{(1,1)}$ labeled by the rank-two antisymmetric tensor representation of $s u(N)$ can then be determined from the equation

$$
G_{(1)} \circ G_{(1)}=G_{(2)}+\frac{\theta(q, p) \theta\left(t^{2}, p\right)}{\theta(t, p) \theta(q t, p)} G_{(1,1)} .
$$

By this method, we find that the elliptic difference operator $G_{(1,1)}$ for the antisymmetric tensor representation is given by

$$
G_{(1,1)} \cdot \mathcal{I}\left(a_{i}\right)=t^{-1} \sum_{j_{1}<j_{2}} \prod_{k \neq\left\{j_{1}, j_{2}\right\}} \frac{\theta\left(\frac{t}{q} a_{j_{1}} / a_{k}, p\right) \theta\left(\frac{t}{q} a_{j_{2}} / a_{k}, p\right)}{\theta\left(a_{k} / a_{j_{1}}, p\right) \theta\left(a_{k} / a_{j_{2}}, p\right)} \mathcal{I}\left(q^{\frac{2}{N}-\delta_{i,\left\{j_{1}, j_{2}\right\}}} a_{i}\right) .
$$

The term in the sum labeled by $j_{1}<j_{2}$ corresponds to the weight $\lambda=h_{j_{1}}+h_{j_{2}}$ in the antisymmetric tensor representation $(1,1)$.

Next, we determine the difference operator $G_{(2,1)}$ from the equation

$$
\left(G_{(2)} \circ G_{(1)}\right) \cdot \mathcal{I}=G_{(3)} \cdot \mathcal{I}+\frac{\theta\left(q^{2}, p\right) \theta\left(q t^{2}, p\right)}{\theta(q t, p) \theta\left(q^{2} t, p\right)} G_{(2,1)} \cdot \mathcal{I}
$$

where

$$
\mathcal{N}_{(2),(1)}^{(2,1)}(p, q, t)=\frac{\theta\left(q^{2}, p\right) \theta\left(q t^{2}, p\right)}{\theta(q t, p) \theta\left(q^{2} t, p\right)}
$$

is the elliptic lift of the $(q, t)$-deformed Littlewood-Richardson coefficient $\mathcal{N}_{(2),(1)}{ }^{(2,1)}(q, t)$.

We verify that the difference operator $G_{(2,1)}$ can indeed be written as a sum over the weights $\lambda=\sum_{i} m_{i} h_{i}$ with $\sum_{i} m_{i}=3$, i.e. as a sum over the weights in the representation labeled by the Young diagram $(2,1)$. These weights can be divided into two groups. The weights $\left\{m_{i_{1}}=m_{i_{2}}=m_{i_{3}}=1\right\}$ occur with multiplicity two, whereas the weights $\left\{m_{j_{1}}=\right.$ $\left.2, m_{j_{2}}=1\right\}$ occur with multiplicity one.

We then expand the resulting operator to lowest order in $p$, read off its elliptic lift and check this in an expansion in $p$. For instance, for $s u(3)$ we find that

$$
\begin{aligned}
G_{(2,1)} \cdot \mathcal{I}\left(a_{1}, a_{2}, a_{3}\right)= & \sum_{\sigma \in S_{3}} C_{210}\left(a_{\sigma(1)}, a_{\sigma(2)}, a_{\sigma(3)}\right) \mathcal{I}\left(\frac{a_{\sigma(1)}}{q}, a_{\sigma(2)}, q a_{\sigma(3)}\right) \\
& +C_{111}\left(a_{1}, a_{2}, a_{3}\right) \mathcal{I}\left(a_{1}, a_{2}, a_{3}\right) .
\end{aligned}
$$


The first group of terms in this sum correspond to weights $\lambda=2 h_{\sigma(1)}+h_{\sigma(2)}$ that occur with multiplicity one. These terms are given by a single product over ratios of theta-functions:

$$
C_{210}\left(a_{\sigma(1)}, a_{\sigma(2)}, a_{\sigma(3)}\right)=t^{-2} \frac{\theta\left(\frac{t a_{\sigma(1)}}{q a_{\sigma(2)}}, p\right) \theta\left(\frac{t a_{\sigma(1)}}{q a_{\sigma(3)}}, p\right) \theta\left(\frac{t a_{\sigma(2)}}{q a_{\sigma(3)}}, p\right) \theta\left(\frac{t a_{\sigma(1)}}{q^{2} a_{\sigma(3)}}, p\right)}{\theta\left(\frac{a_{\sigma(2)}}{a_{\sigma(1)}}, p\right) \theta\left(\frac{a_{\sigma(3)}}{a_{\sigma(1)}}, p\right) \theta\left(\frac{a_{\sigma(3)}}{a_{\sigma(2)}}, p\right) \theta\left(\frac{q a_{\sigma(3)}}{a_{\sigma(1)}}, p\right)} .
$$

The last term corresponds to the weight $\lambda=h_{1}+h_{2}+h_{3}$, which occurs with multiplicity two. Its contribution is given by

$$
\begin{aligned}
& C_{111}\left(a_{1}, a_{2}, a_{3}\right)=-t^{-3} \frac{\theta(t, p) \theta\left(q^{2} t, p\right)}{\theta\left(q^{-1}, p\right) \theta\left(q t^{2}, p\right)} \\
& \times\left(\sum_{\sigma \in S_{3}} \frac{\theta\left(\frac{t a_{\sigma(1)}}{a_{\sigma(2)}}, p\right) \theta\left(\frac{t a_{\sigma(2)}}{a_{\sigma(1)}}, p\right) \theta\left(\frac{t a_{\sigma(1)}}{a_{\sigma(3)}}, p\right) \theta\left(\frac{t a_{\sigma(2)}}{a_{\sigma(3)}}, p\right) \theta\left(\frac{t a_{\sigma(3)}}{q a_{\sigma(1)}}, p\right) \theta\left(\frac{t a_{\sigma(3)}}{q a_{\sigma(2)}}, p\right) \theta\left(\frac{a_{\sigma(2)}}{q a_{\sigma(1)}}, p\right) \theta\left(\frac{a_{\sigma(3)}}{a_{\sigma(1)}}, p\right) \theta\left(\frac{q a_{\sigma(1)}}{a_{\sigma(3)}}, p\right) \theta\left(\frac{a_{\sigma(3)}}{a_{\sigma(2)}}, p\right) \theta\left(\frac{q a_{\sigma(2)}}{a_{\sigma(3)}}, p\right)}{\theta\left(\frac{a_{(1)}}{a_{(1)}}, p\right) \theta(p)}\right. \\
& \left.+\frac{\theta\left(\frac{1}{t}, p\right)^{2} \theta\left(q^{3}, p\right)}{\theta(q, p)^{2} \theta\left(\frac{1}{q t^{2}}, p\right)} \frac{\theta\left(\frac{t a_{1}}{a_{2}}, p\right) \theta\left(\frac{t a_{2}}{a_{1}}, p\right) \theta\left(\frac{t a_{1}}{a_{3}}, p\right) \theta\left(\frac{t a_{2}}{a_{3}}, p\right) \theta\left(\frac{t a_{3}}{a_{1}}, p\right) \theta\left(\frac{t a_{3}}{a_{2}}, p\right)}{\left.\theta a_{1}, p\right) \theta\left(\frac{a_{2}}{q a_{1}}, p\right) \theta\left(\frac{q a_{3}}{a_{1}}, p\right) \theta\left(\frac{q a_{1}}{a_{3}}, p\right) \theta\left(\frac{q a_{3}}{a_{2}}, p\right) \theta\left(\frac{q a_{2}}{a_{3}}, p\right)}\right) .
\end{aligned}
$$

The last term in this expression is invariant itself under permutations of $a_{1}, a_{2}$ and $a_{3}$, the first six terms permute into each other.

Continuing this strategy, one can systematically find the elliptic difference operators for any given representation $R$ and perform consistency checks on it. We have explicitly computed all $s u(3)$ and $s u(4)$ difference operators labeled by Young diagrams with up to four boxes. From these results we infer that the difference operator $G_{\left(1^{r}\right)}$, corresponding to the rank $r$ antisymmetric representation of $s u(N)$, is given by

$$
G_{\left(1^{r}\right)} \cdot \mathcal{I}\left(a_{i}\right)=t^{r(r-N) / 2} \sum_{|I|=r} \prod_{\substack{j \in I \\ k \notin I}} \frac{\theta\left(\frac{t}{q} a_{j} / a_{k}, p\right)}{\theta\left(a_{k} / a_{j}, p\right)} \mathcal{I}\left(q^{\frac{r}{N}-\delta_{i, I}} a_{i}\right),
$$

where the summation is over subsets $I \subset\{1, \ldots, N\}$ of length $|I|=r$ and where the symbol $\delta_{i, I}$ is one if $i \in I$ and zero if $i \notin I$. As we will show in more detail in the next section, these operators are related by conjugation to the Hamiltonians of the elliptic Ruijsenaars-Schneider model.

\subsection{Properties of difference operators}

Let us summarize a few properties of the resulting difference operators $G_{R}$ :

- They are formally self-adjoint respect to the vectormultiplet measure $\Delta(a) \mathcal{I}_{V}(a)$ on the unit circle $|a|=1$.

- The composition $G_{R_{1}} \circ G_{R_{2}}$ is commutative.

- The difference operators $G_{R}$ obey the algebra

$$
G_{R_{1}} \circ G_{R_{2}}=\sum_{R_{3}} \mathcal{N}_{R_{1}, R_{2}}{ }^{R_{3}} G_{R_{3}}
$$

where the coefficients $\mathcal{N}_{R_{1}, R_{2}}{ }^{R_{3}}$ are elliptic lifts of the $(q, t)$-deformed LittlewoodRichardson coefficients. 
- They can be expanded as

$$
G_{R} \cdot \mathcal{I}\left(a_{i}\right)=\sum_{\lambda} C_{R, \lambda}\left(p, q, t, a_{j}\right) \mathcal{I}\left(q^{-\left(\lambda, h_{i}\right)} a_{i}\right)
$$

where the summation is over weights $\lambda$ in the representation $R$.

While we have not found a closed expression for the coefficients $C_{R, \lambda}\left(p, q, t, a_{j}\right)$, it may be useful to point out the following structures:

- For general values of the fugacities, $C_{R, \lambda} \neq C_{R^{\prime}, \lambda}$ when $\lambda$ is a weight of two different representations $R$ and $R^{\prime}$. Only in the Schur limit $q=t$ do the coefficients $C_{R, \lambda}$ depend only on the weight $\lambda$.

- The coefficients $C_{R, \lambda}$ are given by a single ratio of theta-functions when the weight $\lambda$ occurs in the representation $R$ with multiplicity one. If $\lambda$ occurs with higher multiplicity the coefficient $C_{R, \lambda}$ is a sum of ratios of theta-functions.

- If $\lambda$ and $\lambda^{\prime}$ are in the same Weyl orbit, the coefficients $C_{R, \lambda}$ and $C_{R, \lambda^{\prime}}$ are related by a permutation of fugacities $a_{i}$.

- If $\lambda$ is in the Weyl orbit of the highest weight in the representation $R$, the coefficient $C_{R, \lambda}$ does not contain theta-functions that are independent of the fugacities $a_{j}$.

- If $\lambda$ is not in the Weyl orbit of the highest weight in the representation $R$, the coefficient $C_{R, \lambda}$ does contain a ratio of such theta-functions that are independent of $a_{j}$. This ratio can be obtained as an elliptic lift of the corresponding coefficient for the Macdonald polynomial $P_{R}(q, t)$.

We also note that the elliptic lift of the $(q, t)$-deformed Littlewood-Richardson coefficients $\mathcal{N}_{R_{1}, R_{2}}{ }^{R_{3}}(p, q, t)$ have the same number of terms in the numerator and denominator. Moreover, when $q=t$ these terms all cancel each other. This implies that the elliptic algebra reduces to the Schur algebra when $q=t$. In this limit all coefficients $C_{R, \lambda}(p, q, t)$ reduce to a single product $C_{\lambda}(q)$ depending only on the weight $\lambda$ as found previously in [17].

\subsection{Two-dimensional worldvolume theory}

So far, we have constructed an algebra of operators $G_{R}$ that compute the superconformal index in the presence of a set of surface defects labeled by irreducible representations $R$ of $s u(N)$. The operators $G_{(r)}$, labeled by symmetric tensor representations, were found in an infinite tension limit of two-dimensional half-BPS vortices. The operators for the remaining representations were obtained in a canonical way by completing the algebra.

An alternative and more direct way to define surface defects is by coupling to twodimensional degrees of freedom on the supported surface. For instance, consider a twodimensional $\mathcal{N}=(2,2)$ gauge theory with flavor symmetry group $\mathrm{SU}(N)$. This twodimensional theory can be coupled to the four-dimensional theory by gauging the $2 \mathrm{~d}$ flavor symmetry using the restriction of a $4 \mathrm{~d}$ dynamical or background $\mathrm{SU}(N)$ vectormultiplet 


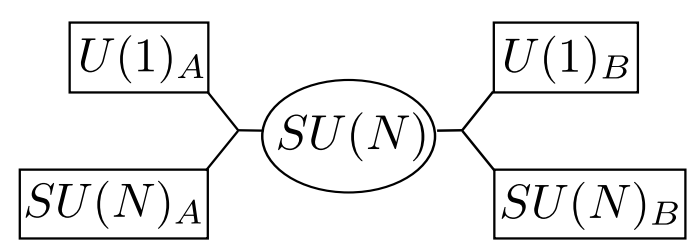

Figure 5. Linear quiver description of $\mathcal{N}=2$ superconformal QCD. The flavor symmetry group of each set of $N$ hypers is enhanced to $\mathrm{U}(N)$. This splits into an $\mathrm{SU}(N)$ plus a diagonal $\mathrm{U}(1)$ flavor symmetry group.

to the surface $S$. A large class of half-BPS surface defects defined in this way have been studied in $[19,20]$.

The superconformal index of four-dimensional linear $\mathcal{N}=2$ quiver theories in the presence of such surface defects can be found by combining the two-dimensional elliptic genus with the four-dimensional superconformal index [21]. Let us consider this combined index in a few examples of surface defects in $\mathcal{N}=2$ superconformal QCD, i.e. a fourdimensional $\mathrm{SU}(N)$ gauge theory coupled to $2 N$ hypermultiplets.

Before introducing surface defects, let us remind ourselves that $\mathcal{N}=2$ superconformal QCD has a dual description as a degeneration limit of a Riemann surface with two simple and two full punctures. Equivalently, its matter content can be read off from a linear quiver, see figure 5 . The manifest global symmetry in this presentation is

$$
\mathrm{SU}(N)_{A} \times \mathrm{SU}(N)_{B} \times \mathrm{U}(1)_{A} \times \mathrm{U}(1)_{B} .
$$

If we denote the corresponding fugacities by $\left(a_{i}, b_{i}, x, y\right)$, the superconformal index of superconformal QCD is

$$
\int \Delta\left(z_{j}\right) \mathcal{I}_{V}\left(z_{j}\right) \mathcal{I}_{H}\left(z_{j}^{-1}, a_{i}, x\right) \mathcal{I}_{H}\left(z_{j}, b_{i}, y\right)
$$

Notice that we could have equivalently considered the same theory with a $\mathrm{U}(N)$ gauge group, since the center of mass U(1) decouples in the IR.

Let us now add two-dimensional degrees of freedom to the four-dimensional superconformal QCD theory with gauge group $\mathrm{U}(N)$. We give two examples whose $2 \mathrm{~d}-4 \mathrm{~d}$ quiver descriptions are shown in figure 6.

As a first example, we consider a two-dimensional $\mathcal{N}=(2,2)$ gauge theory with gauge group $\mathrm{U}(r)$ coupled to $N$ fundamental and $N$ anti-fundamental chiral fields. The twodimensional flavor symmetry group is thus $\mathrm{U}(N)_{f} \times \mathrm{U}(N)_{a}$. We couple the $N$ fundamental chirals to the $\mathrm{U}(N)$ gauge symmetry, and the $N$ anti-fundamental chirals to the $\mathrm{SU}(N)_{B} \times$ $\mathrm{U}(1)_{B}$ global symmetry, as described in [21]. The resulting quiver is illustrated on top in figure 6 . The superconformal index of the resulting $2 \mathrm{~d}-4 \mathrm{~d}$ system is

$$
\int \Delta\left(z_{j}\right) \mathcal{I}_{V}\left(z_{j}\right) \mathcal{I}_{H}\left(z_{j}^{-1}, a_{i}, x\right)\left(\mathcal{O}_{r} \cdot \mathcal{I}_{H}\left(z_{j}, b_{i}, y\right)\right),
$$

where the operator $\mathcal{O}_{r}$ acts as

$$
\mathcal{O}_{r} \cdot \mathcal{I}\left(z_{i}\right)=\sum_{|I|=r} \prod_{\substack{j \in I \\ k \notin I}} \frac{\theta\left(\frac{t}{q} z_{j} / z_{k}, p\right)}{\theta\left(z_{k} / z_{j}, p\right)} \mathcal{I}\left(q^{-\delta_{i, I}} z_{i}\right)
$$



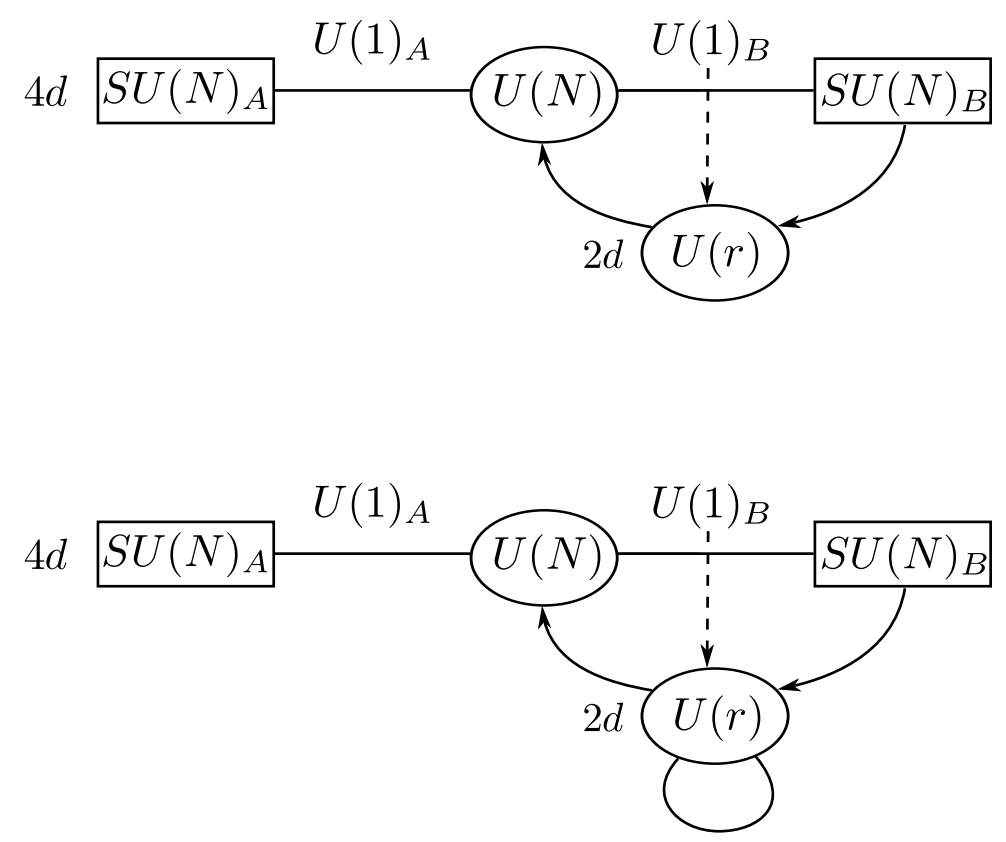

Figure 6. On top (bottom): 2d-4d quiver description of a fully antisymmetric (symmetric) surface defect in $\mathcal{N}=2$ superconformal QCD.

on the fugacities $z_{i}$ of the $\mathrm{U}(N)$ gauge symmetry.

The terms in the above expression are in one-to-one correspondence with the $\left(\begin{array}{l}N \\ r\end{array}\right)$ Higgs branch vacua of the two-dimensional theory, in which certain components of the chiral fields get a vacuum expectation value. Each term in equation (2.47) can be interpreted as computing the index of the $2 \mathrm{~d}-4 \mathrm{~d}$ system in one of these vacua.

The operator $\mathcal{O}_{r}$ agrees with the elliptic difference operator $G_{\left(1^{r}\right)}$ labeled by the antisymmetric tensor representation $\left(1^{r}\right)$ of rank $r$ up to an overall fractional shift by $q^{\frac{r}{N}}$. Since the shifts $z_{i} \mapsto q^{-\delta_{i, I}} z_{i}$ do not preserve the condition $\prod_{j} z_{j}=1$, the fugacities $z_{j}$ should really be interpreted as $\mathrm{U}(N)$ (instead of $\mathrm{SU}(N)$ ) fugacities. To find the exact operators $G_{\left(1^{r}\right)}$, however, we would need to find a system that couples the same two-dimensional degrees of freedom to a $4 \mathrm{~d}$ theory with genuine $\mathrm{SU}(N)$ symmetry groups. This is for instance required to understand surface defects in the four-dimensional $\mathcal{T}_{N}$ theory, whose flavor symmetry groups cannot be enlarged to $\mathrm{U}(N)$.

As a second example, we add a chiral field in the adjoint representation to the twodimensional $\mathcal{N}=(2,2)$ theory that we considered before. The quiver description can be found on the bottom of figure 6 . The presence of the adjoint field drastically changes the vacuum structure, which is mirrored in the expression for the superconformal index. The index of the $2 \mathrm{~d}-4 \mathrm{~d}$ system is the same as before, except that the operator $\mathcal{O}_{r}$ now acts as

$$
\mathcal{O}_{r} \cdot \mathcal{I}\left(z_{i}\right)=\sum_{\sum_{j=1}^{N} m_{j}=r} \prod_{i, j=1}^{N}\left[\prod_{m=0}^{m_{j}-1} \frac{\theta\left(q^{m+m_{j}-m_{i}} t z_{i} / z_{j}, p\right)}{\theta\left(q^{m-m_{j}} z_{j} / z_{i}, p\right)}\right] \mathcal{I}\left(q^{-m_{i}} z_{i}\right)
$$

This expression coincides with the operator $G_{(r)}$ associated to the symmetric representation $(r)$ of rank $r$, as was already noted in [21], except that the fractional shift $q^{\frac{r}{N}}$ is again missing. 
For the symmetric as well as the antisymmetric tensor representations the twodimensional degrees of freedom on the surface defect introduced by the operators $G_{R}$, can thus be identified with certain two-dimensional $\mathcal{N}=(2,2)$ gauge theories, up to some shifts.

It would be interesting to observe whether the $S^{2}$ partition function of these $\mathcal{N}=(2,2)$ theories can be obtained from Toda correlators with degenerate vertex operators labeled by highest weights of the symmetric and antisymmetric tensor representations. This has been demonstrated for the fundamental representation in [31] (see also [18, 32]).

\section{Two-dimensional TQFT and Verlinde algebra}

In this section we identify the difference operators $G_{R}$ with local operators in a topological quantum field theory (TQFT) of the Riemann surface $C$. In the case $p=0$, this can be identified with an analytic continuation of refined Chern-Simons theory on $S^{1} \times C$ and the relevant local operators arise from Wilson loops in the representation $R$ and wrapping the $S^{1}$.

\subsection{TQFT structure of the superconformal index}

Recall that for any superconformal field theory of class $\mathcal{S}$ the superconformal index is independent of marginal couplings and hence of the complex structure of the Riemann surface $C$. This suggests that the superconformal index of these theories has a dual description as a two-dimensional TQFT on the Riemann surface $C$ [12]. In the Schur limit (when $p \rightarrow 0$ and $q=t$ ), the TQFT has been identified as $q$-deformed Yang-Mills theory on $C$ in the zero area limit [14], or equivalently as an analytic continuation of Chern-Simons theory on $C \times S^{1}$. This picture can be extended to the Macdonald limit $(p \rightarrow 0)$ when the superconformal index has a dual description as an analytic continuation of refined Chern-Simons theory on $C \times S^{1}[22]$.

In order to verify the above relation, it is necessary to extract a certain function $K(a)$ from the superconformal index for each $\mathrm{SU}(N)$ flavor puncture. In what follows, we define the normalized index $\mathcal{I}^{(n)}$ through the equation

$$
\mathcal{I}(a, b, \ldots)=(K(a) K(b) \cdots) \mathcal{I}^{(n)}(a, b, \ldots),
$$

where

$$
K(a)=\prod_{i \neq j}^{N} \Gamma\left(t a_{i} / a_{j}, p, q\right)
$$

The normalized index $\mathcal{I}^{(n)}$ is now gauged using the measure

$$
\Delta^{(n)}(a)=K(a) \Delta(a)=\frac{1}{N !}\left(\frac{(p, p)(q, q)}{\Gamma(t, p, q)}\right)^{N-1} \prod_{i \neq j} \frac{\Gamma\left(t a_{i} / a_{j}, p, q\right)}{\Gamma\left(a_{i} / a_{j}, p, q\right)} .
$$

The difference operators $\bar{G}_{R}$ acting on the normalized index are thus obtained by conjugation

$$
\bar{G}_{R}=\frac{1}{K(a)}\left(G_{R} \cdot K(a)\right) .
$$


This conjugation leaves the algebra of difference operators unchanged. After a long, yet straightforward, computation we find that the conjugated operators for the fully symmetric representations $R=(r)$ are given by

$$
\bar{G}_{(r)} \cdot \mathcal{I}^{(n)}\left(a_{i}\right)=\mathcal{N}_{r} \sum_{\sum_{j=1}^{N} m_{j}=r} \prod_{i, j=1}^{N} \prod_{m=0}^{m_{j}-1} \frac{\theta\left(t q^{m} a_{i} / a_{j}, p\right)}{\theta\left(q^{m-m_{i}} a_{i} / a_{j}, p\right)} \mathcal{I}^{(n)}\left(q^{\frac{r}{N}-m_{i}} a_{i}\right)
$$

while those for the fully antisymmetric representations $R=\left(1^{r}\right)$ are

$$
\bar{G}_{\left(1^{r}\right)} \cdot \mathcal{I}^{(n)}\left(a_{i}\right)=t^{r(r-N) / 2} \sum_{|I|=r} \prod_{\substack{k \in I \\ j \notin I}} \frac{\theta\left(t a_{j} / a_{k}, p\right)}{\theta\left(a_{j} / a_{k}, p\right)} \mathcal{I}^{(n)}\left(q^{\frac{r}{N}-\delta_{i, I}} a_{i}\right),
$$

where the summation is over all subsets $I \subset\{1,2, \ldots, N\}$ of length $r$. Comparing with (2.14) and (2.42) noting the reflection property $\theta(z, p)=\theta(p / z, p)$ we see that the effect of the conjugation is simply to interchange $t \leftrightarrow p q / t$. In summary, we have found that

$$
\bar{G}_{R}(p, q, t, a)=G_{R}\left(p, q, \frac{p q}{t}, a\right) .
$$

Remarkably, the conjugated antisymmetric tensor operators $\bar{G}_{\left(1^{r}\right)}$ are precisely the Hamiltonians of the elliptic Ruijsenaars-Schneider model, extending the observation made in [10].

We will assume that the difference operators $\bar{G}_{R}$ admit a complete set of eigenfunctions $\left\{\psi_{S}\left(a_{i}\right)\right\}$, indexed by irreducible representations $S$ of $s u(N)$, which are orthogonal with respect to the measure $\Delta^{(n)}(a)$ and have non-degenerate eigenvalues $E_{(R)}^{S}$. In fact, the eigenfunctions are determined by the fully antisymmetric operators $\bar{G}_{\left(1^{r}\right)}$. With the help of these eigenfunctions $\left\{\psi_{S}\left(a_{i}\right)\right\}$ the TQFT structure of the superconformal index can be made very explicit [10].

Consider for instance the sphere with three maximal punctures. The corresponding four-dimensional conformal field theory is known as $\mathcal{T}_{N}$. It has at least $\mathrm{SU}(N)^{3}$ flavor symmetry. Write the normalized superconformal index of the $\mathcal{T}_{N}$ theory as

$$
\mathcal{I}^{(n)}\left(a_{i}, b_{i}, c_{i}\right),
$$

where the parameters $a_{i}, b_{i}$ and $c_{i}$ are three sets of fugacities dual to the maximal torus of the $\mathrm{SU}(N)$ flavor symmetries. This superconformal index can be expanded in terms of the set of eigenfunctions $\left\{\psi_{S}\left(a_{i}\right)\right\}$ as

$$
\mathcal{I}^{(n)}\left(a_{i}, b_{i}, c_{i}\right)=\sum_{S_{1}, S_{2}, S_{3}} C_{S_{1}, S_{2}, S_{3}} \psi_{S_{1}}\left(a_{i}\right) \psi_{S_{2}}\left(b_{i}\right) \psi_{S_{3}}\left(c_{i}\right),
$$

where $C_{S_{1}, S_{2}, S_{3}}$ are the structure constants of the two-dimensional TQFT. If we impose that acting with any one of the operators $\bar{G}_{\left(1^{r}\right)}$ gives the same result, and assume that the eigenvalues are non-degenerate then the superconformal index is in fact diagonal in this basis

$$
\mathcal{I}^{(n)}\left(a_{i}, b_{i}, c_{i}\right)=\sum_{S} C_{S} \psi_{S}\left(a_{i}\right) \psi_{S}\left(b_{i}\right) \psi_{S}\left(c_{i}\right)
$$




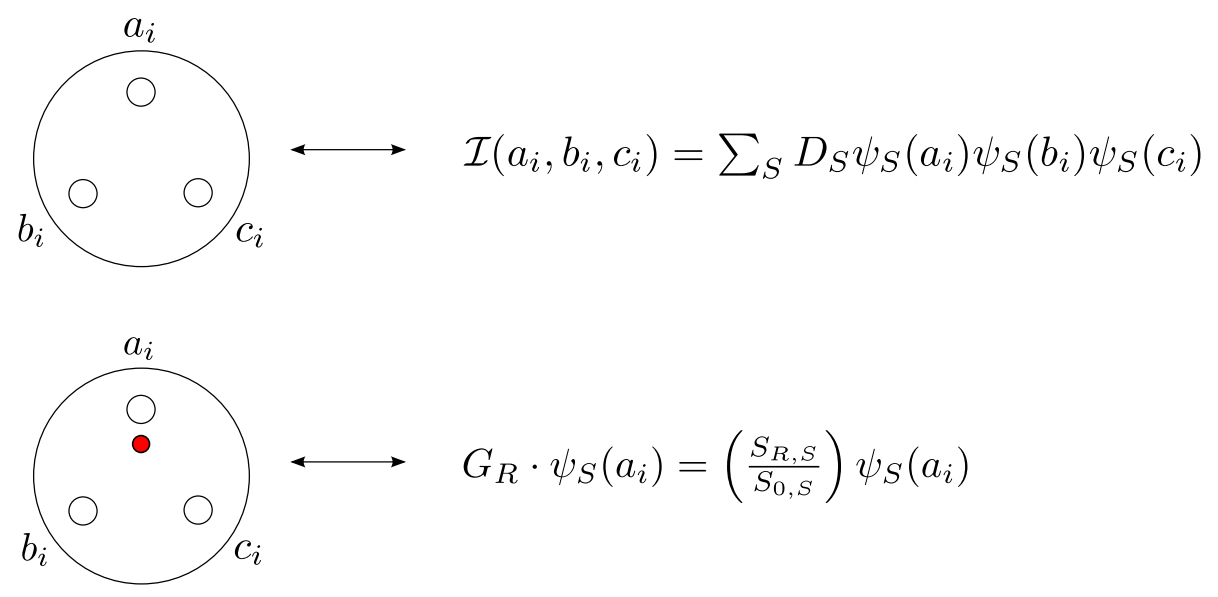

Figure 7. The superconformal index can be written as a TQFT correlator. This correlator is diagonal in the eigenfunctions $\psi_{S}\left(a_{i}\right)$ of the difference operators $G_{R}$.

This is illustrated in figure 7 for the case of a sphere with three punctures. As explained in [10], the constants $C_{S}$ can be found by comparing two degeneration limits of the $N+1$ punctured sphere with $N-1$ maximal and two minimal punctures.

In the remainder of this section we will restrict ourselves to the Macdonald slice $(p, q, t)=(0, q, t)$. In this limit, the antisymmetric difference operators $\bar{G}_{\left(1^{r}\right)}$ turn into the Macdonald operators

$$
\bar{G}_{\left(1^{r}\right)} \cdot \mathcal{I}^{(n)}\left(a_{i}\right)=t^{r(r-N) / 2} \sum_{|I|=r} \prod_{\substack{k \in I \\ j \notin I}} \frac{\left(1-q a_{j} / a_{k}, p\right)}{\left(1-a_{j} / a_{k}, p\right)} \mathcal{I}^{(n)}\left(q^{\frac{r}{N}-\delta_{i, I}} a_{i}\right)
$$

while the normalized vectormultiplet measure becomes

$$
\Delta^{(n)}(a)=\prod_{i \neq j} \frac{\left(1-t a_{i} / a_{j}, q\right)}{\left(1-a_{i} / a_{j}, q\right)}
$$

and coincides with the standard Macdonald measure.

The operators $\bar{G}_{\left(1^{r}\right)}$ are self-adjoint with respect to this measure on the unit circle $|a|=1$ and their common eigenfunctions are the Macdonald polynomials $P_{S}\left(a_{i} ; q, t\right)$, which are labeled by irreducible representations of $s u(N)$. They are by construction orthogonal with respect to the measure $\Delta^{(n)}(a)$ and are normalized such that

$$
P_{S}\left(a_{i} ; q, t\right)=\chi_{S}\left(a_{i}\right)+\sum_{T<S} c_{S, T}(q, t) \chi_{T}\left(a_{i}\right) .
$$

In this equation, the $c_{S, T}$ are rational functions of $q$ and $t$ that are uniquely fixed by ensuring that $P_{S}\left(a_{i} ; q, t\right)$ is an eigenfunction of the Macdonald operators $\bar{G}_{\left(1^{r}\right)}$ for $r=1, \ldots, N-1$. In this limit the structure constants $C_{S}$ are given by

$$
C_{S}=\frac{1}{S_{0, S}}
$$

where $S_{R, S}$ is an analytic continuation of the modular S-matrix of refined Chern-Simons theory. 


\subsubsection{Operator algebra from Macdonald polynomials}

The Macdonald polynomials obey

$$
P_{S_{1}} \cdot P_{S_{2}}=\sum_{S} \mathcal{N}_{S_{1}, S_{2}}{ }^{S_{3}} P_{S_{3}}
$$

where $\mathcal{N}_{S_{1}, S_{2}}{ }^{S_{3}}$ are the $(q, t)$-deformed Littlewood-Richardson coefficients. Remarkably, we have found that the conjugated difference operators $\bar{G}_{R}$ obey the same algebra. Let us try to understand this fact.

Consider for instance the case $N=2$. The eigenvalues of the difference operators $\bar{G}_{r}$ can be computed from explicit formulae that we have found. By experimentation, we find that they are given by

$$
\bar{G}_{r_{1}} \cdot P_{r_{2}}(a)=\frac{S_{r_{1}, r_{2}}}{S_{0, r_{2}}} P_{r_{2}},
$$

where $S_{r_{1}, r_{2}}$ is an analytic continuation of the modular S-matrix of refined Chern-Simons theory (see appendix A for the construction of this quantity). This formula can be proven by a lengthy computation using the residue construction, in which the eigenvalue is given by

$$
\frac{\operatorname{Res}_{a=t^{\frac{1}{2}} q^{\frac{r_{1}}{2}}} \frac{1}{a} P_{r_{2}}(a)}{\operatorname{Res}_{a=t^{\frac{1}{2}}} \frac{1}{a} P_{r_{2}}(a)}=\frac{S_{r_{1}, r_{2}}}{S_{0, r_{2}}} .
$$

This S-matrix is known to obey the $(q, t)$-deformed Verlinde formula

$$
S_{r_{1}, s} \cdot S_{r_{2}, s}=S_{0, s} \sum_{r} \mathcal{N}_{r_{1}, r_{2}}^{r} S_{r, s}
$$

Let us now act with the composition of the operators $\bar{G}_{r_{1}}$ and $\bar{G}_{r_{2}}$ on the Macdonald polynomial $P_{s}$ and apply the refined Verlinde formula

$$
\begin{aligned}
\left(\bar{G}_{r_{1}} \circ \bar{G}_{r_{2}}\right) \cdot P_{s} & =\frac{S_{r_{1}, s}}{S_{0, s}} \frac{S_{r_{2}, s}}{S_{0, s}} P_{s} \\
& =\sum_{r} \mathcal{N}_{r_{1}, r_{2}}{ }^{r} \frac{S_{r, s}}{S_{0, s}} P_{s} \\
& =\sum_{r} \mathcal{N}_{r_{1}, r_{2}}{ }^{r} \bar{G}_{r} \cdot P_{s} .
\end{aligned}
$$

Since the Macdonald polynomials form a complete basis of symmetric functions, we find that the structure constants of the difference operator algebra are indeed the $(q, t)$-deformed Littlewood-Richardson coefficients.

Similarly, we have verified that the generalized difference operators $\bar{G}_{R}$, labeled by irreducible representations $R$ of $s u(N)$, satisfy the eigenvalue equation

$$
\bar{G}_{R_{1}} \cdot P_{R_{2}}=\frac{S_{R_{1}, R_{2}}}{S_{0, R_{2}}} P_{R_{2}}
$$

in the Macdonald slice. 


\subsection{Wilson loops in refined Chern-Simons theory}

In the Macdonald slice the superconformal index is dual to an analytic continuation of the refined Chern-Simons theory on $S^{1} \times C$. Similar to the discussion in [17] we can identify the surface defect operators $\bar{G}_{R}$ in this refined Chern-Simons theory as the Wilson loop operator

$$
\mathcal{O}_{R}=\operatorname{Tr}_{R} \mathrm{P} \exp \left(i \oint_{S^{1}} A\right)
$$

in the representation $R$ wrapping the $S^{1}$. This is of course a local operator from the perspective of the two-dimensional TQFT on $C$, in accordance with our expectations from six-dimensional engineering.

Correlation functions of this operator are independent of its position on $C$ and simply insert a ratio $S_{R, S} / S_{0, S}$ in the sum over representations $S$ is any correlator. For example, inserting the operator $\mathcal{O}_{R}$ in a correlator on the three-punctured sphere is computed as

$$
\left\langle\mathcal{O}_{R}\right\rangle_{0,3}=\sum_{S} \frac{P_{S}\left(a_{1}\right) P_{S}\left(a_{2}\right) P_{S}\left(a_{3}\right)}{S_{S, 0}} \frac{S_{R, S}}{S_{0, S}}
$$

where $S_{S, R}$ is an analytic continuation of the modular S-matrix of refined Chern-Simons theory. Hence inserting the local operator $\mathcal{O}_{R}$ in a TQFT correlation function is equivalent to acting on any of the punctures with the difference operator $\bar{G}_{R}$.

Moreover, from the $(q, t)$-deformed Verlinde formula

$$
S_{R_{1}, S} \cdot S_{R_{2}, S}=S_{0, S} \sum_{R} \mathcal{N}_{R_{1}, R_{2}}{ }^{R} S_{R, S}
$$

we derive the operator product expansion

$$
\mathcal{O}_{R_{1}} \cdot \mathcal{O}_{R_{2}}=\sum_{R} \mathcal{N}_{R_{1}, R_{2}}{ }^{R} \mathcal{O}_{R}
$$

where $\mathcal{N}_{R_{1}, R_{2}}{ }^{R}$ are the analytically continued $(q, t)$-deformed Littlewood-Richardson coefficients. Thus in the Macdonald limit, the algebra of the difference operators $G_{R}$ is equivalent to the Verlinde algebra in refined Chern-Simons theory on $S^{1} \times C$.

The general superconformal index could be taken to define a $(p, q, t)$-deformed YangMills theory on $C$, whose structure constants are given in terms of the eigenfunctions $\psi_{R}\left(a_{i} ; p, q, t\right)$ of the elliptic difference operators. The difference operators satisfy an algebra whose structure constants $\mathcal{N}_{R_{1}, R_{2}}{ }^{R_{3}}$ are elliptic functions. It would be fascinating to understand this theory.

\section{Algebra of three-dimensional line defects}

In section 2, we constructed the superconformal index of $\mathcal{N}=2$ theories on $S^{1} \times S^{3}$ in the presence of certain surface defects supported on $S^{1} \times S^{1}$. These surface defects were labeled by an irreducible representation $R$ of $s u(N)$ and could be added to any superconformal theory with an $\mathrm{SU}(N)$ flavor symmetry. In this section, we consider the reduction of the 
four-dimensional superconformal index to a partition function on a squashed three-sphere $S^{3}$, following [23-25]. In this limit, the surface defects become codimension-two defects in the three-dimensional theory wrapping an $S^{1} \subset S^{3}$.

For four-dimensional theories of class $\mathcal{S}$, upon dimensionally reducing on $S^{1}$ the theory flows to an $\mathcal{N}=4$ superconformal field theory in three-dimensions. Moreover, this has a mirror description in terms of a star-shaped quiver theory [26]. It is expected that the surface defects introduced by the difference operators $G_{R}$ become supersymmetric Wilson loops in representation $R$ for the central node of this star-shaped quiver upon dimensional reduction. We demonstrate this explicitly for antisymmetric tensor representations $R=\left(1^{r}\right)$ and the case of a round three-sphere. For non-minuscule representations $R$, however, we find that the difference operators $G_{R}$ introduce a linear combination of Wilson loops in irreducible representations $S$ with $|S| \leq|R|$.

\subsection{From superconformal index to $3 \mathrm{~d}$ partition function}

The four-dimensional superconformal index on $S^{1} \times S^{3}$ can be reduced to a partition function on the squashed three-sphere $S^{3}$, as demonstrated in [23-25]. This limit is taken by parametrizing the fugacities by

$$
p=e^{-\beta b^{-1}}, \quad q=e^{-\beta b}, \quad t=e^{-\beta \epsilon}, \quad a_{j}=e^{-i \beta x_{j}},
$$

with $\beta>0$ and then taking the limit $\beta \rightarrow 0^{+}$. Here we have introduced the convenient notation $\epsilon=\frac{q}{2}+i m$ where $q=b+b^{-1}$.

The real parameter $b>0$ encodes the geometry of the three-sphere, defined by the embedding

$$
b^{-2}|z|^{2}+b^{2}|w|^{2}=1
$$

into $\mathbb{C}^{2}$ with complex coordinates $(z, w)$. The parameters $x_{i}$ with $\sum_{i=1}^{N} x_{i}=0$ are real mass parameters for the global $\mathrm{SU}(N)$ symmetry that is inherited by the three-dimensional theory. It is convenient to repackage the components $x_{j}$ into a vector $x$ such that $x_{j}=\left(x, h_{j}\right)$. In addition, the real parameter $m$ gives a mass to the adjoint chiral multiplet inside the background $\mathcal{N}=4$ vectormultiplet, breaking the supersymmetry to $\mathcal{N}=2$ in three dimensions.

Let us consider two important examples. Firstly, the three-dimensional limit (4.1) of the superconformal index of a free hypermultiplet is given by

$$
\mathcal{Z}_{H}(x)=S_{b}\left(\frac{\epsilon}{2} \pm i x\right) .
$$

Secondly, the superconformal index of an $\mathrm{SU}(N)$ vectormultiplet combined with the Haar measure becomes the partition function of a three-dimensional $\mathcal{N}=4$ vectormultiplet

$$
\mathcal{Z}_{V}(x)=\prod_{i<j}^{N} 2 \sin \left(i \pi b^{ \pm} x_{i j}\right) \mathcal{K}(x)
$$

where

$$
\mathcal{K}(x)=\frac{1}{S_{b}\left(\epsilon^{*}\right)} \prod_{i, j=1}^{N} S_{b}\left(\epsilon^{*}+i x_{i j}\right)
$$


where we have denoted $\epsilon^{*}=\frac{q}{2}-i m$. Note that $\mathcal{K}(x)$ is the contribution from the $\mathcal{N}=2$ adjoint chiral multiplet inside the three-dimensional $\mathcal{N}=4$ vectormultiplet and cancel in pairs in the limit $m \rightarrow 0$. We use the double sine function that obeys the difference equation $S_{b}\left(x+b^{ \pm}\right)=2 \sin \left(\pi b^{ \pm} x\right) S_{b}(x)$ and the reflection property $S_{b}(x) S_{b}(q-x)=1$. Further properties of this special function can be found in appendix $\mathrm{C}$.

Let us now consider the three-dimensional limit of the difference operators $G_{R}$ that introduce surface defects into the four-dimensional $\mathcal{N}=2$ theory. The three-dimensional limit can be evaluated using the fact that the ratio of theta-functions with a common second argument reduces to a ratio of sine-functions,

$$
\frac{\theta\left(e^{\alpha \rho}, e^{\beta \rho}\right)}{\theta\left(e^{\gamma \rho}, e^{\beta \rho}\right)} \stackrel{\rho \rightarrow 0}{\longrightarrow} \frac{\sin (\pi \alpha / \beta)}{\sin (\pi \gamma / \beta)} .
$$

Let us first consider the difference operator $G_{(1)}$ labeled by the fundamental representation of $s u(N)$. In four dimensions this operator is given by

$$
G_{(1)} \cdot \mathcal{I}\left(a_{i}, \ldots\right)=\sum_{j=1}^{N} \prod_{k \neq j}^{N} \frac{\theta\left(\frac{t}{q} a_{j} / a_{k}, p\right)}{\theta\left(a_{k} / a_{j}, p\right)} \mathcal{I}\left(q^{\frac{1}{N}-\delta_{k, i}} a_{i}, \ldots\right)
$$

up to some overall $t$-dependent factor. Applying the above formula to each term, we find that the three-dimensional limit of the fundamental difference operator $G_{(1)}$ acts on the three-dimensional partition function $\mathcal{Z}(x, \ldots)$ as

$$
G_{(1)}^{(3 \mathrm{~d})} \cdot \mathcal{Z}(x, \ldots)=\sum_{j=1}^{N} \prod_{k \neq j}^{N} \frac{\sin \pi b\left(\epsilon^{*}-i x_{j k}\right)}{\sin \pi b\left(-i x_{j k}\right)} \mathcal{Z}\left(x+i b h_{j}, \ldots\right),
$$

where we use the shorthand $x_{j k}=x_{j}-x_{k}$. We also recall that the weights $h_{i}$ obey $\left(h_{i}, h_{j}\right)=\delta_{i j}-1 / N$.

Let us now extend this computation to the rank $r$ antisymmetric tensor representation $\left(1^{r}\right)$ of $s u(N)$. In section 2 we found that up to a power of $t$ the corresponding difference operator is

$$
G_{\left(1^{r}\right)} \cdot \mathcal{I}\left(a_{i}\right)=\sum_{|I|=r} \prod_{\substack{j \in I \\ k \notin I}} \frac{\theta\left(\frac{t}{q} a_{j} / a_{k}, p\right)}{\theta\left(a_{k} / a_{j}, p\right)} \mathcal{I}\left(q^{\frac{r}{N}-\delta_{i, I}} a_{i}\right),
$$

where the summation is over subsets $I \subset\{1, \ldots, N\}$ of length $|I|=r$ and where the symbol $\delta_{i, I}$ is one if $i \in I$ and zero if $i \notin I$. In the three-dimensional limit, we obtain the operator

$$
G_{\left(1^{r}\right)}^{(3 \mathrm{~d})} \cdot \mathcal{Z}(x)=\sum_{|I|=r} \prod_{\substack{j \in I \\ k \notin I}} \frac{\sin \pi b\left(\epsilon^{*}-i x_{j k}\right)}{\sin \pi b\left(-i x_{j k}\right)} \mathcal{Z}\left(x+i b \sum_{j \in I} h_{j}\right)
$$

acting on the squashed three-sphere partition function $\mathcal{Z}(x)$. Similar computations can be performed for the four-dimensional difference operators $G_{R}$ corresponding to any irreducible representation $R$ of $s u(N)$. 


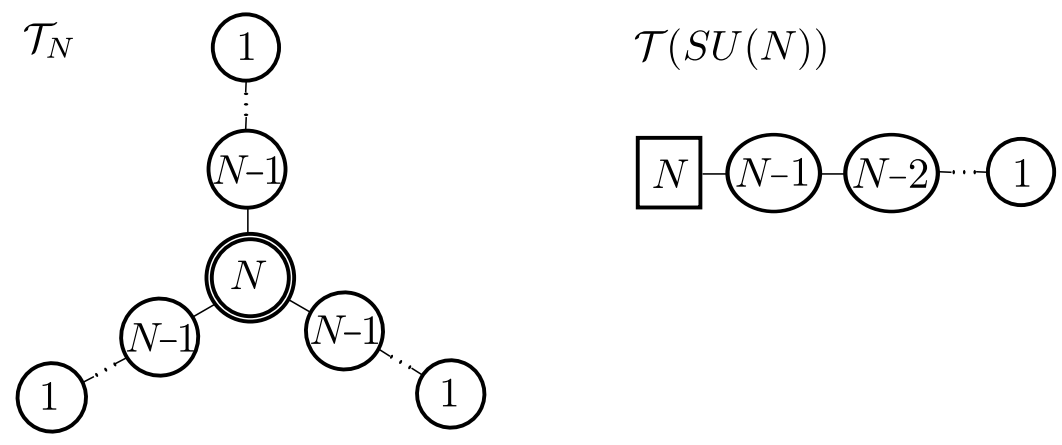

Figure 8. Left: star-shaped quiver corresponding to the mirror of the three-dimensional $\mathcal{T}_{N}$ theory. Right: linear quiver description of the three-dimensional $\mathcal{T}(\mathrm{SU}(N))$ theory.

The dimensionally reduced operators $G_{R}^{(3 \mathrm{~d})}$ have similar properties to their fourdimensional ancestors. The adjoint operator of $G_{R}^{(3 \mathrm{~d})}$ with respect to the three-dimensional $\mathcal{N}=4$ vectormultiplet measure (4.4) on $\mathbb{R}^{N-1}$,

$$
\left\langle f_{1}, f_{2}\right\rangle=\int\left[\frac{\mathrm{d}^{N-1} x}{N !} \prod_{i<j}^{N} 2 \sin \left(i \pi b^{ \pm} x_{i j}\right) \mathcal{K}(x)\right] \cdot f_{1}(x) f_{2}(x),
$$

is given by the operator $G_{R^{*}}^{(3 \mathrm{~d})}$ for the conjugate representation $R^{*}$. Furthermore, the operators $G_{R}^{(3 \mathrm{~d})}$ generate a commutative algebra that can be derived by applying the limit (4.1) to the general structure constants $\mathcal{N}_{R_{1}, R_{2}}{ }^{R_{3}}(p, q, t)$ that we found in the four-dimensional case.

Finally, in section 3.1 we pointed out that the four-dimensional difference operators with the replacement $t \rightarrow p q / t$ were related by a similarity transformation. In the threedimensional limit, this corresponds to $m \rightarrow-m$ or quivalently $\epsilon \rightarrow \epsilon^{*}$. Thus, either by direct computation or by taking the three-dimensional limit of the the result in section 3.1 we find that

$$
G_{R}^{(3 d)}(x)=\frac{1}{\mathcal{K}(x)} \cdot \bar{G}_{R}^{(3 d)}(x) \cdot \mathcal{K}(x)
$$

where the operator $\bar{G}_{R}^{(3 d)}(x)$ is related to $G_{R}^{(3 d)}(x)$ by the replacement $\epsilon \rightarrow \epsilon^{*}$ and $\mathcal{K}(x)$ is the partition function of an adjoint $\mathcal{N}=2$ chiral multiplet inside an $\mathcal{N}=4$ vectormultiplet. A consequence is that the eigenfunctions of the two sets of operators are proportional.

\subsection{Wilson loops in 3d star-shaped quivers}

Since the dimensional reduction is performed along a circle on which the surface defect is supported, we expect that the difference operators (4.8) and (4.10) introduce defects in the three-dimensional theory supported on the circle $|z|^{2}=b^{2}$. In the following we will perform indirect checks of this prediction by exploiting three-dimensional mirror symmetry to relate these defects to supersymmetric Wilson loops.

Upon dimensional reduction, a four-dimensional $\mathcal{N}=2$ theory of class $\mathcal{S}$ flows to an $\mathcal{N}=4$ superconformal field theory in three dimensions, which is related by mirror symmetry to a star-shaped quiver gauge theory [26]. For example, the mirror of the threedimensional reduction of the $\mathcal{T}_{N}$ theory is given by a star-shaped quiver with three legs, 
shown in figure 8. More generally, each full puncture in four dimensions gives rise to one copy of the linear quiver illustrated on the right in figure 8 . The corresponding field theory is known as the $\mathcal{T}(\mathrm{SU}(N))$ theory [33,34]. This theory contains $\mathcal{N}=4$ vectormultiplets for the gauge groups $\mathrm{U}(1), \ldots, \mathrm{U}(N-1)$. These gauge groups are coupled linearly through $\mathrm{U}(k)-\mathrm{U}(k+1)$ bifundamental hypermultiplets. Lastly, there are $N$ hypermultiplets in the fundamental representation of the largest gauge group $\mathrm{U}(N-1)$.

The $\mathcal{T}(\mathrm{SU}(N))$ theory has manifest $\mathrm{SU}(N)$ Higgs branch symmetry acting on the $N$ hypermultiplets whose mass parameters are denoted by the vector $x$. In addition, we can introduce $N-1$ Fayet-Illiopoulos parameters $t_{1}, \ldots, t_{N-1}$, which are mass parameters for the topological $\mathrm{U}(1)$ symmetries associated to each node of the quiver. Let us express these parameters in terms of a new vector $y$ such that $t_{k}=y_{k}-y_{k+1}$. Let us denote the partition function of $\mathcal{T}(\mathrm{SU}(N))$ by $\mathcal{Z}_{\epsilon}(x, y)$. This is expected to be invariant under exchanging $x \leftrightarrow y$ and $\epsilon \leftrightarrow \epsilon^{*}$. This symmetry reflects an enhancement of the Coulomb branch symmetry to ${ }^{L} \mathrm{SU}(N)$ in the infrared, as well as the presence of mirror symmetry exchanging $\mathrm{SU}(N) \leftrightarrow{ }^{L} \mathrm{SU}(N)$.

The partition function of any three-dimensional theory of class $\mathcal{S}$ with full punctures can then be constructed according to the star-shaped quiver description. For example, the partition function of the three-dimensional mass-deformed $\mathcal{T}_{N}$ theory is given by

$$
\mathcal{Z}_{\mathcal{T}_{N}}(x, y, z)=\int \frac{d^{N-1} w}{N !} \mathcal{Z}_{V}(w) \mathcal{Z}_{\epsilon}(w, x) \mathcal{Z}_{\epsilon}(w, y) \mathcal{Z}_{\epsilon}(w, z)
$$

where $\mathcal{Z}_{V}(w)$ is the partition function of an $\mathcal{N}=4$ vectormultiplet. This multiplet is used to gauge the diagonal combination of $\mathrm{SU}(N)$ Higgs branch symmetries.

Let us consider the action of the three-dimensional operators on the partition function $\mathcal{Z}(x, y, z)$. Similar as in four dimensions, the result should be independent of which puncture we act on. This condition is guaranteed if the partition function of each quiver tail $\mathcal{T}(\mathrm{SU}(N))$ is an eigenfunction of the operator $G_{\left(1^{r}\right)}^{(3 \mathrm{~d})}$. We will now show that in fact we have

$$
G_{\left(1^{r}\right)}^{(3 \mathrm{~d})}(y) \cdot \mathcal{Z}_{\epsilon}(x, y)=W_{\left(1^{r}\right)}(x) \mathcal{Z}_{\epsilon}(x, y)
$$

where

$$
W_{\left(1^{r}\right)}(x)=\sum_{j_{1}<\ldots<j_{r}} e^{-2 \pi b\left(x_{j_{1}}+\cdots+x_{j_{r}}\right)}
$$

is the expectation value of a supersymmetric Wilson loop in the rank $r$ antisymmetric representation of the $\mathrm{SU}(N)$ flavor symmetry ${ }^{3}$ with mass parameter $x$.

But let us first remark that equation (4.14) tells us that introducing a background defect in the $\mathcal{T}(\mathrm{SU}(N))$ theory for the Coulomb branch symmetry, through the operator $G_{\left(1^{r}\right)}^{(3 \mathrm{~d})}(y)$, is equivalent to introducing a background Wilson loop $W_{\left(1^{r}\right)}(x)$ for the Higgs branch symmetry. This means that mirror symmetry interchanges the defects introduced by the operators $G_{\left(1^{r}\right)}^{(3 \mathrm{~d})}$ and supersymmetric Wilson loops in the $r$-th antisymmetric representation of $\mathrm{SU}(N)$. In the context of the mirror description of the three-dimensional

\footnotetext{
${ }^{3}$ Recall that Wilson loops are labeled by irreducible representations of the gauge group. Here we use that each irreducible representation $R$ of $s u(N)$ corresponds to an irreducible representation $R$ of $\mathrm{SU}(N)$, and vice versa.
} 
$\mathcal{T}_{N}$ theory, the operators $G_{\left(1^{r}\right)}^{(3 \mathrm{~d})}$ therefore introduce a dynamical Wilson loop for the central node of the star-shaped quiver theory.

\subsection{1 $\mathcal{T}(\mathrm{SU}(2))$}

We first show equation (4.14) in complete generality for the partition function of the mass deformed $\mathcal{T}(\mathrm{SU}(2))$ theory on a squashed three-sphere. This partition function is given by

$$
\mathcal{Z}_{\epsilon}(x, y)=\frac{1}{S_{b}\left(\epsilon^{*}\right)} \int d z \mathcal{Q}(x, z) e^{4 \pi i y z}
$$

where

$$
\mathcal{Q}(x, z)=\frac{S_{b}\left(\frac{\epsilon^{*}}{2} \pm i x-i z\right)}{S_{b}\left(q-\frac{\epsilon^{*}}{2} \pm i x-i z\right)},
$$

$x$ is the $\mathrm{SU}(2)$ mass parameter and $y$ the FI parameter and the contour is given by $z \in$ $\mathbb{R}+i \epsilon$ with $\epsilon>0$. Note that the $\mathcal{N}=2$ mass deformation $m$ in the hypermultiplet contribution appears with the opposite sign compared to equation (4.3). The reason is that after dimensional reduction, there is a mirror symmetry required to reach the starshaped quiver description.

It is expected that the partition function has the following properties

$$
\begin{aligned}
\mathcal{Z}_{\epsilon}(x, y) & =\mathcal{Z}_{\epsilon}(-x, y)=\mathcal{Z}_{\epsilon}(x,-y) \\
\mathcal{Z}_{\epsilon}(x, y) & =\mathcal{Z}_{\epsilon^{*}}(y, x) \\
G_{(1)}^{(3 d)}(y) \mathcal{Z}_{\epsilon}(x, y) & =W_{(1)}(x) \mathcal{Z}_{\epsilon}(x, y),
\end{aligned}
$$

where

$$
G_{(1)}^{(3 d)}(x)=\frac{\sin \pi b\left(\epsilon^{*}-2 i x\right)}{\sin \pi b(-2 i x)} e^{\frac{i b}{2} \partial_{x}}+\frac{\sin \pi b\left(\epsilon^{*}+2 i x\right)}{\sin \pi b(2 i x)} e^{-\frac{i b}{2} \partial_{x}}
$$

is the fundamental difference operator (4.8) when $N=2$, and $W_{(1)}(x)=e^{2 \pi b x}+e^{-2 \pi b x}$ is the fundamental Wilson loop expectation value.

The first line of equation (4.18) represents the enhancement of the Higgs and Coulomb branch symmetry to $\mathrm{SU}(2) \times{ }^{L} \mathrm{SU}(2)$ in the infrared. The second line illustrates the mirror symmetry of the mass-deformed $\mathcal{T}(\mathrm{SU}(2))$ theory. These properties were demonstrated in [35]. Here we would like to prove the final line of equation (4.18). Using mirror symmetry this line is equivalent to

$$
\bar{G}_{(1)}^{(3 \mathrm{~d})}(x) \mathcal{Z}_{\epsilon}(x, y)=W_{(1)}(y) \mathcal{Z}_{\epsilon}(x, y),
$$

where $\bar{G}_{(1)}^{(3 \mathrm{~d})}(x)$ is obtained from $G_{(1)}^{(3 \mathrm{~d})}(x)$ by the replacement $m \rightarrow-m$. Let us prove the intertwining property in this equivalent form.

As a preliminary step, we derive a few properties of the function $\mathcal{Q}(x, z)$ defined in equation (4.17). From the difference equation and the reflection property obeyed by the double sine function $S_{b}(x)$, it is straightforward to show that

$$
e^{i b \partial_{z}} \mathcal{Q}(x, z)=\frac{\sin \pi b\left(\frac{\epsilon^{*}}{2} \pm i x-i z\right)}{\sin \pi b\left(q-\frac{\epsilon^{*}}{2} \pm i x-i z\right)} \mathcal{Q}(x, z)
$$


and

$$
\begin{array}{r}
e^{\frac{i b}{2} \partial_{x}} \mathcal{Q}(x, z)=e^{-\frac{i b}{2} \partial_{z}}\left[\frac{\sin \pi b\left(\frac{\epsilon^{*}}{2}-i x-i z\right)}{\sin \pi b\left(q-\frac{\epsilon^{*}}{2}-i x-i z\right)} \mathcal{Q}(x, z)\right] \\
e^{-\frac{i b}{2} \partial_{x}} \mathcal{Q}(x, z)=e^{-\frac{i b}{2} \partial_{z}}\left[\frac{\sin \pi b\left(\frac{\epsilon^{*}}{2}+i x-i z\right)}{\sin \pi b\left(q-\frac{\epsilon^{*}}{2}+i x-i z\right)} \mathcal{Q}(x, z)\right],
\end{array}
$$

where we have used the notation $\epsilon=\frac{q}{2}+i m$. Using these results we can now compute the action of the difference operator $\bar{G}_{(1)}^{(3 \mathrm{~d})}(x)$ on this function,

$$
\begin{aligned}
& \bar{G}_{(1)}^{(3 \mathrm{~d})}(x) \cdot \mathcal{Q}(x, z)=e^{-\frac{i b}{2} \partial_{z}} {\left[\frac{\sin \pi b(\epsilon-2 i x)}{\sin \pi b(-2 i x)} \frac{\sin \pi b\left(\frac{\epsilon^{*}}{2}-i x-i z\right)}{\sin \pi b\left(q-\frac{\epsilon^{*}}{2}-i x-i z\right)}\right.} \\
&\left.+\frac{\sin \pi b(\epsilon+2 i x)}{\sin \pi b(2 i x)} \frac{\sin \pi b\left(\frac{\epsilon^{*}}{2}+i x-i z\right)}{\sin \pi b\left(q-\frac{\epsilon^{*}}{2}+i x-i z\right)}\right] \mathcal{Q}(x, z) \\
&=e^{-\frac{i b}{2} \partial_{z}}\left[1+\frac{\sin \pi b\left(\frac{\epsilon^{*}}{2} \pm i x-i z\right)}{\sin \pi b\left(q-\frac{\epsilon^{*}}{2} \pm i x-i z\right)}\right] \mathcal{Q}(x, z) \\
&=\left(e^{\frac{i b}{2} \partial_{z}}+e^{-\frac{i b}{2} \partial_{z}}\right) \mathcal{Q}(z, x)
\end{aligned}
$$

In going from the first to the second line we have applied a simple trigonometric identity.

Armed with this result, we now consider the action of the difference operator $\bar{G}_{(1)}^{(3 \mathrm{~d})}(x)$ on the full partition function (4.16) of the $\mathcal{T}(\mathrm{SU}(2))$ theory. The difference operator can be brought inside the integral to act on $\mathcal{Q}(x, z)$ as in equation (4.24). By shifting the contour of integration by $z \rightarrow z \pm \frac{i b}{2}$, we find

$$
\begin{aligned}
\bar{G}_{(1)}^{(3 \mathrm{~d})}(x) \cdot \mathcal{Z}_{\epsilon}(x, y) & =\frac{1}{S_{b}\left(\frac{q}{2}-i m\right)} \int d z\left[\left(e^{\frac{i b}{2} \partial_{z}}+e^{-\frac{i b}{2} \partial_{z}}\right) \mathcal{Q}(x, z)\right] e^{4 \pi i y z} \\
& =\frac{1}{S_{b}\left(\frac{q}{2}-i m\right)} \int \mathcal{Q}(x, z)\left[\left(e^{\frac{i b}{2} \partial_{z}}+e^{-\frac{i b}{2} \partial_{z}}\right) e^{4 \pi i y z}\right] \\
& =W_{(1)}(y) \mathcal{Z}_{\epsilon}(x, y) .
\end{aligned}
$$

Using the analytic structure of the double sine function $S_{b}(x)$, it is straightforward to check that no poles are crossed in shifting the contours provided the mass are real. Now, applying mirror symmetry we have

$$
G_{(1)}^{(3 \mathrm{~d})}(y) \mathcal{Z}_{\epsilon}(x, y)=W_{(1)}(x) \mathcal{Z}_{\epsilon}(x, y),
$$

which is the required result.

An important consequence of this result, together with the similarity transformation (4.12) relating the operators $G_{(1)}^{(3 d)}$ and $\bar{G}_{(1)}^{(3 d)}$, is that the partition function of mass deformed $\mathcal{T}(\mathrm{SU}(2))$ theory obeys

$$
\mathcal{Z}_{\epsilon^{*}}(x, y)=\mathcal{K}(y) \mathcal{Z}_{\epsilon}(x, y)
$$

which is rather non-obvious from the integral representation. 


\subsection{2 $\mathcal{T}(\mathrm{SU}(N))$}

Let us now consider equation (4.14) for the general $\mathcal{T}(\mathrm{SU}(N))$ theory. In this case, we simplify the problem and prove a weaker result by taking the limit of $\mathcal{N}=4$ supersymmetry $(m=0)$ and a round three-sphere $(b=0)$.

In this limit, the operators for the fully antisymmetric representations are given by

$$
G_{\left(1^{r}\right)}^{(3 \mathrm{~d})} \cdot \mathcal{Z}(x)=(-1)^{r(N-r)} \sum_{j_{1}<\cdots<j_{r}} \mathcal{Z}\left(x+i\left(h_{j_{1}}+\cdots+h_{j_{r}}\right)\right) .
$$

Hence, up to a sign, the operators are simply a sum of shift operators with coefficient 1 with the shifts determined by the weights of the representation $\left(1^{r}\right)$.

Furthermore, the zero mode integrals in the partition function of the $\mathcal{T}(\mathrm{SU}(N))$ theory can be performed explicitly [36, 37]. The result is ${ }^{4}$

$$
\mathcal{Z}(x, y)=\frac{\sum_{\rho \in S_{N}}(-1)^{\rho} e^{2 \pi i \sum_{j=1}^{N} x_{\rho(j)} y_{j}}}{\prod_{i<j} 2 \sinh \pi\left(x_{i}-x_{j}\right) 2 \sinh \pi\left(y_{i}-y_{j}\right)},
$$

where the summation in the numerator is over the Weyl group $S_{N}$ of permutations of $\{1, \ldots, N\}$. Mirror symmetry $x \leftrightarrow y$ in this case follows from the identity

$$
\sum_{\rho \in S^{N}}(-1)^{\rho} e^{2 \pi i \sum_{j=1}^{N} x_{\rho(j)} y_{j}}=\sum_{\rho \in S^{N}}(-1)^{\rho} e^{2 \pi i \sum_{j=1}^{N} x_{j} y_{\rho(j)}} .
$$

Thus there is a vast simplification in the limit $b=1$ and $m=0$.

Let us now act with the operator $G_{\left(1^{r}\right)}^{(3 \mathrm{~d})}$ on the partition function $\mathcal{Z}(x, y)$. First, note that each term in the operator $G_{\left(1^{r}\right)}^{(3 \mathrm{~d})}$ leaves the denominator invariant up to a factor $(-1)^{r(N-r)}$ which cancels the overall sign in the operator. Thus we can concentrate on the numerator of $\mathcal{Z}(x, y)$ and find

$$
G_{\left(1^{r}\right)}^{(3 \mathrm{~d})}(y) \cdot \sum_{\rho \in S^{N}}(-1)^{\rho} e^{2 \pi i \sum_{j=1}^{N} y_{\rho(j)} x_{j}}=\sum_{|I|=r}\left[\sum_{\rho \in S^{N}}(-1)^{\rho} e^{2 \pi i \sum_{k=1}^{N} y_{\rho(k)} x_{k}} e^{-2 \pi \sum_{\rho(i) \in I} x_{i}}\right] .
$$

Imposing the condition $\sum_{i=1}^{N} x_{i}=0$ leads to vanishing overall background shifts.

Label the subsets $I \subset\{1, \ldots, N\}$ with $|I|=r$ by $I_{\ell}$ for $1 \leq \ell \leq N_{r}=\left(\begin{array}{c}N \\ r\end{array}\right)$. Furthermore split $S^{N}$ into $\mathbb{Z}_{N_{r}} \otimes\left(S^{N-r} \otimes S^{r}\right)$, where $S^{(N-r)} \otimes S^{r}$ gives all the permutations satisfying $\rho\left(I_{\ell}\right)=I$ for fixed $I$ and $\ell$, and $\mathbb{Z}_{N_{r}}$ gives the different choices of $\ell$ (or $I$ ). Since this splitting is an isomorphism, ${ }^{5}$ we can write the sum over all $\rho \in S^{N}$ as a double sum over sets $I$ with $|I|=r$ and permutations in $S^{N-r} \otimes S^{r}$ preserving $\rho\left(I_{\ell}\right)=I$. With that in mind, we can rewrite the above as

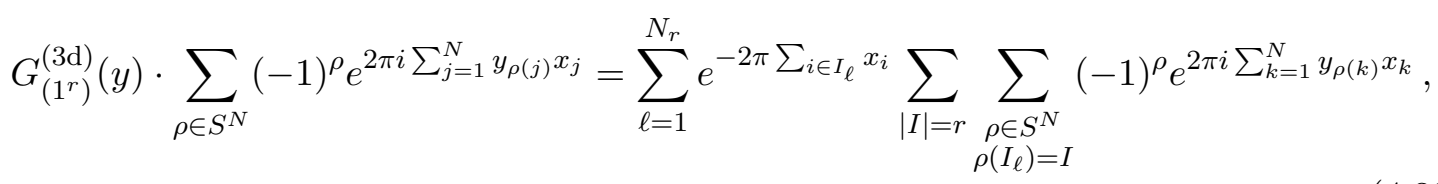

\footnotetext{
${ }^{4}$ Here we drop the subscript $m$ on the partition function of $\mathcal{T}(\mathrm{SU}(N))$ because we have set $m=0$.

${ }^{5}$ Any $\rho \in S^{N}$ can be uniquely characterized by $\rho\left(\sigma\left(I_{\ell}\right)\right)=\pi(I)$ for a unique $I_{\ell}$ or $I$ and $\sigma \in S^{r}, \pi \in S^{N-r}$ permutations of $I, \mathbb{Z}_{N} \backslash I$ respectively.
} 
which is equal to the Wilson loop vacuum expectation value $W_{\left(1^{r}\right)}(x)$ times the numerator of $\mathcal{Z}(x, y)$. The eigenvalue of the operator $G_{\left(1^{r}\right)}^{(3 \mathrm{~d})}$ acting on the full partition function $\mathcal{Z}(x, y)$ is thus precisely the localization expression for a supersymmetric Wilson loop in the antisymmetric representation $\left(1^{r}\right)$ of $\mathrm{SU}(N)$.

\subsection{Three-dimensional algebra}

In the above, we have shown that the defect operator $G_{\left(1^{r}\right)}^{(3 \mathrm{~d})}$ is dual to a Wilson loop in the rank $r$ antisymmetric representation of $\mathrm{SU}(N)$ under mirror symmetry. This turns out not to be the case for non-minuscule representations.

One immediate way to see this is the following. Denote the operator that $i s$ exactly dual to a Wilson loop in the representation $R$ by $\tilde{G}_{R}^{(3 \mathrm{~d})}$. The operators $\tilde{G}_{R}^{(3 \mathrm{~d})}$ must obey the algebra

$$
\tilde{G}_{R_{1}}^{(3 \mathrm{~d})} \circ \tilde{G}_{R_{2}}^{(3 \mathrm{~d})}=\sum_{R_{1}} N_{R_{1}, R_{2}}^{R_{3}} \tilde{G}_{R_{3}}^{(3 \mathrm{~d})},
$$

where $N_{R_{1}, R_{2}}{ }^{R_{3}}$ are the (plain) Littlewood-Richardson coefficients. Indeed, the supersymmetric Wilson loops are characters and hence obey this algebra. Instead, the elliptic Littlewood-Richardson coefficients $\mathcal{N}_{R_{1}, R_{2}}{ }^{R_{3}}(p, q, t)$ reduce in general to non-integer coefficients in three dimensions.

For example, let us consider $\mathrm{SU}(2)$ and the composition of two operators in the fundamental representation. In the three-dimensional limit we find that

$$
G_{(1)}^{(3 \mathrm{~d})} \circ G_{(1)}^{(3 \mathrm{~d})}=G_{(2)}^{(3 \mathrm{~d})}+\left(\frac{1}{2 \cos \left(\pi b^{2}\right)-1}-1\right) G_{(0)}^{(3 \mathrm{~d})} .
$$

Since $\tilde{G}_{(1)}^{(3 \mathrm{~d})}=G_{(1)}^{(3 \mathrm{~d})}$ and $\tilde{G}_{(0)}^{(3 \mathrm{~d})}=G_{(0)}^{(3 \mathrm{~d})}=1$, we read off from equation (4.33) that

$$
\tilde{G}_{(2)}^{(3 \mathrm{~d})}=G_{(2)}^{(3 \mathrm{~d})}+\left(\frac{1}{2 \cos \left(\pi b^{2}\right)-1}-2\right) G_{(0)}^{(3 \mathrm{~d})}
$$

The operator $\tilde{G}_{(2)}^{(3 \mathrm{~d})}$ that is dual to a Wilson loop thus differs from the difference operator $G_{(2)}^{(3 d)}$ by lower order contributions.

In general, the relation between the operators $G_{R}^{(3 \mathrm{~d})}$ appearing in the vortex construction and the operators $\tilde{G}_{R}^{(3 \mathrm{~d})}$ that are exactly dual to Wilson loops in the three-dimensional limit is given by

$$
\tilde{G}_{R}^{(3 \mathrm{~d})}=G_{R}^{(3 \mathrm{~d})}+\sum_{|S|<|R|} c_{S} G_{S}^{(3 \mathrm{~d})}
$$

Even though the difference operators $G_{R}^{(3 \mathrm{~d})}$ are thus not exactly dual to Wilson loops, this is merely an invertible linear transformation on the algebra that these operators obey.

The original basis of operators $G_{R}$ appears to be more fundamental from a fourdimensional perspective, since in the limit $p \rightarrow 0$ they are precisely dual to Wilson loop operators in refined Chern-Simons theory on $S^{1} \times C$. On the other hand, in the threedimensional limit, the basis $\tilde{G}_{R}^{(3 \mathrm{~d})}$ seems more fundamental since it is dual to a basis of Wilson loop operators in the star-shaped quiver theories. 


\section{5 't Hooft loops in the four-dimensional $\mathcal{N}=2^{*}$ theory}

In this section, we realize the mass-deformed theory $\mathcal{T}(\mathrm{SU}(N))$ on a squashed three-sphere as an S-duality domain wall in four-dimensional $\mathcal{N}=2^{*}$ theory on an ellipsoid, as described in $[35,38]$. We then use this observation to interpret the three-dimensional difference operators $G_{R}^{(3 \mathrm{~d})}$ as operators that introduce supersymmetric 't Hooft loops in the $\mathcal{N}=2^{*}$ theory partition function on a four-sphere.

The four-dimensional $\mathcal{N}=2^{*}$ theory can also be obtained by compactifying the sixdimensional $(2,0)$ theory of type $A_{N-1}$ on a torus with a simple puncture. A consequence of this construction is that via the AGT correspondence $[7,8]$, the four-sphere partition function of the $\mathcal{N}=2^{*}$ theory can also be computed as a correlation function in Liouville or Toda CFT on the punctured torus. The difference operators $G_{R}^{(3 \mathrm{~d})}$ can then be interpreted as Verlinde loop operators that act on suitably normalized Virasoro or $W_{N}$-algebra conformal blocks on a punctured torus.

\subsection{Four-sphere partition function}

The exact partition function of $\mathcal{N}=2$ supersymmetric gauge theories on an ellipsoid has been computed by supersymmetric localization in [39], extending the computation of Pestun for the round four-sphere $S^{4}$ [40]. The ellipsoid geometry can be embedded into five-dimensional Euclidean space as

$$
x_{0}^{2}+\frac{1}{b^{2}}\left(x_{1}^{2}+x_{2}^{2}\right)+b^{2}\left(x_{3}^{2}+x_{4}^{2}\right)=1,
$$

where $b \in \mathbb{R}_{\geq 0}$ is a real parameter. The equator $\left\{x_{0}=0\right\}$ is identified with the squashed three-sphere geometry considered in the previous section by setting $z=x_{1}+i x_{2}$ and $w=x_{3}+i x_{4}$.

Let us concentrate on the $\mathcal{N}=2^{*}$ theory and denote the real hypermultiplet mass parameter by $m$ and the complexified gauge coupling by $\tau$. The result of the localization computation can be written as a matrix integral

$$
\mathcal{Z}_{S_{b}^{4}}(m, \tau)=\int d a|\mathcal{Z}(a, m ; \tau)|^{2}
$$

over a real slice of the Coulomb branch. In this integral $\mathcal{Z}(a, m ; \tau)$ is the Nekrasov partition function for the four-dimensional $\mathcal{N}=2^{*}$ theory in the Omega-background $\mathbb{R}_{\epsilon_{1}, \epsilon_{2}}^{4}$, with equivariant parameters $\epsilon_{1}=b$ and $\epsilon_{2}=b^{-1}$ [41, 42]. It can be split into a classical, 1-loop and instanton piece as

$$
\mathcal{Z}(a, m ; \tau):=\mathcal{Z}_{\mathrm{cl}}(a ; \tau) \mathcal{Z}_{1-\mathrm{loop}}(a, m) \mathcal{Z}_{\text {inst }}(a, m ; \tau) .
$$

In this paper we advertise an alternative factorization of the ellipsoid partition function $\mathcal{Z}_{S_{b}^{4}}$. We find it insightful to rewrite the matrix integral 5.2 in the form (for a derivation of this representation see appendix $\mathrm{C}$ )

$$
\mathcal{Z}_{S_{b}^{4}}(m ; \tau)=\int d a \mu(a)|\mathcal{G}(a, m ; \tau)|^{2},
$$


where

$$
\mu(a)=\prod_{e>0} 2 \sinh (\pi b(e, a)) 2 \sinh \left(\pi b^{-1}(e, a)\right)
$$

is the Haar measure times the partition function of a three-dimensional $\mathcal{N}=2$ vectormultiplet on the squashed three-sphere at the equator $\left\{x_{0}=0\right\}$.

We expect that the factorization (5.4) has the following interpretation [29]. We can cut the ellipsoid into two half-spheres $\left\{x_{0}>0\right\}$ and $\left\{x_{0}<0\right\}$ and impose Dirichlet boundary conditions on the fields in the $\mathcal{N}=2^{*}$ theory at the boundary $\left\{x_{0}=0\right\}$. This decouples the dynamics on both half-spheres. Restricting the gauge transformations to the identity on the boundary, leaves a flavor symmetry group $\mathrm{SU}(N)$ acting on the values of the fields at $x_{0}=0$. We can reconstruct the partition function of an ellipsoid by inserting a threedimensional $\mathcal{N}=2 \mathrm{SU}(N)$ vectormultiplet on the boundary $\left\{x_{0}=0\right\}$ and gauging the diagonal $\mathrm{SU}(N)$ symmetry. We thus claim that $\mathcal{G}(a, m ; \tau)$ in the matrix integral $(5.4)$ is the partition function of $\mathcal{N}=2^{*}$ theory on the upper half of the ellipsoid $\left\{x_{0}>0\right\}$ with Dirichlet boundary conditions, and similarly for $\overline{\mathcal{G}(a, m ; \tau)}$ on the lower half $\left\{x_{0}<0\right\}$.

Note that $\mathcal{G}(a, m ; \tau)$ can be split into classical, one-loop and instanton contributions just like the Nekrasov partition function in (5.3). Whereas we take its classical and instanton contributions to be the same as those of $\mathcal{Z}(a, m ; \tau)$, i.e. $\mathcal{G}_{\mathrm{cl}}(a ; \tau) \equiv \mathcal{Z}_{\mathrm{cl}}(a ; \tau)$ and $\mathcal{G}_{\text {inst }}(a, m ; \tau) \equiv \mathcal{Z}_{\text {inst }}(a, m ; \tau)$, the one-loop factor $\mathcal{G}_{1-\text { loop }}$ is not canonically determined. We claim that it is fixed by imposing Dirichlet boundary conditions on the half-sphere, in such a way that

$$
\mathcal{G}_{1-\text { loop }}(a, m)=\frac{\prod_{w \in \text { adj }} \Gamma_{b}\left(\frac{q}{2}+i(a, w)+i m\right)}{\prod_{e>0} \Gamma_{b}(q+i(a, e)) \Gamma_{b}(q-i(a, e))},
$$

where $q=b+b^{-1}$ and $\Gamma_{b}(x)$ is the Barnes' double gamma function. The numerator contains the contribution from the vectormultiplet and the denominator that from the adjoint hypermultiplet with mass $m$ in the $\mathcal{N}=2^{*}$ theory.

Let us mention that via the AGT correspondence, in the case $N=2$, this choice is equivalent to a commonly used normalization of Virasoro conformal blocks in Liouville theory, as described for example in [28]. For this choice of normalization, we will show that the expectation values of 't Hooft loop operators in the $\mathcal{N}=2^{*}$ theory are given by acting on $\mathcal{G}(a, m ; \tau)$ with the three-dimensional difference operators $G_{R}^{(3 \mathrm{~d})}$, constructed in section 4 .

\section{$5.2 \quad$ S-duality domain wall}

The three-dimensional theory $\mathcal{T}(\mathrm{SU}(N))$ appears as an S-duality domain wall between two four-dimensional $\mathcal{N}=4 \mathrm{SYM}$ theories with gauge groups $\mathrm{SU}(N)$ and ${ }^{L} \mathrm{SU}(N)$ respectively and equal holomorphic gauge coupling $\tau$ [33,34]. Furthermore, the mass deformation $m$ of the domain wall theory can be identified with the canonical mass deformation of the bulk theory to $\mathcal{N}=2^{*}$ by giving a mass to the adjoint $\mathcal{N}=2$ hypermultiplet.

On the ellipsoid $S_{b}^{4}$, one can introduce the S-duality domain wall at the equator $\left\{x_{0}=0\right\}$ in a way that preserves half of the supersymmetries of the bulk [29]. As 


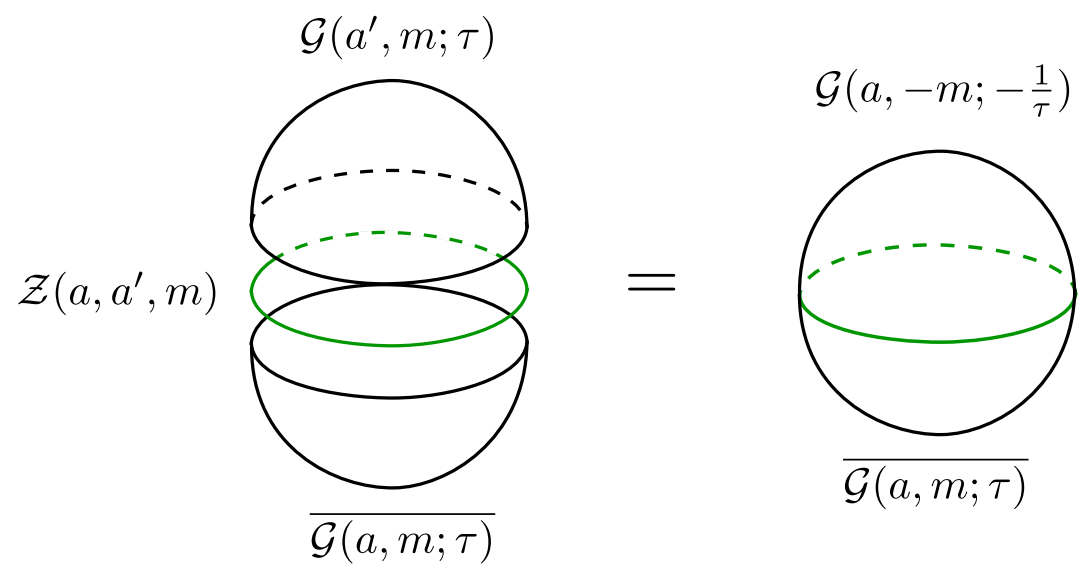

Figure 9. Left: the ellipsoid partition function in the presence of an S-duality domain wall can be constructed by gluing in the domain wall partition function $\mathcal{Z}\left(a, a^{\prime}, m\right)$ in between the half-sphere partition functions $\overline{\mathcal{G}(a, m ; \tau)}$ and $\mathcal{G}\left(a^{\prime}, m ; \tau\right)$, while gauging their flavor symmetries. Right: the same ellipsoid partition function can be constructed by gluing the half-sphere partition functions $\overline{\mathcal{G}(a, m ; \tau)}$ and $\mathcal{G}\left(a,-m ;-\frac{1}{\tau}\right)$.

above, let us assume that the normalized function $\overline{\mathcal{G}(a, m ; \tau)}$ corresponds to the partition function of the $\mathcal{N}=2^{*}$ theory with gauge group $\mathrm{SU}(N)$ on $\left\{x_{0}<0\right\}$ with Dirichlet boundary conditions for the vectormultiplet, and similarly that $\mathcal{G}\left(a^{\prime}, m ; \tau\right)$ corresponds to the partition function of the $\mathcal{N}=2^{*}$ theory with gauge group ${ }^{L} \mathrm{SU}(N)$ on $\left\{x_{0}>0\right\}$. Let us also denote the partition function of the $\mathcal{T}(\mathrm{SU}(N))$ theory on the squashed three-sphere at the equator $\left\{x_{0}=0\right\}$ by $\mathcal{Z}\left(a, a^{\prime}, m\right)$, where $a$ and $a^{\prime}$ are mass parameters for the $\mathrm{SU}(N) \times{ }^{L} \mathrm{SU}(N)$ global symmetry as in section 4 . Then the combined partition function in the presence of the S-duality domain wall is

$$
\int d a d a^{\prime} \mu(a) \mu\left(a^{\prime}\right) \overline{\mathcal{G}(a, m ; \tau)} \mathcal{Z}\left(a, a^{\prime}, m\right) \mathcal{G}\left(a^{\prime}, m ; \tau\right)
$$

where $\mu(a) \mu\left(a^{\prime}\right)$ is the partition function of three-dimensional $\mathcal{N}=2$ vectormultiplets on the equator $\left\{x_{0}=0\right\}$ that gauge the symmetry $\mathrm{SU}(N) \times{ }^{L} \mathrm{SU}(N)$ (see figure 9).

Another interpretation of the same domain wall is as a Janus domain wall interpolating between holomorphic gauge coupling $\tau$ for $\left\{x_{0}<0\right\}$ and $-1 / \tau$ for $\left\{x_{0}>0\right\}$. The two pictures are related by an S-duality transformation of the theory on $\left\{x_{0}>0\right\}$. Another way of saying this is that the partition function $\mathcal{Z}\left(a, a^{\prime}, m\right)$ should be an S-duality kernel relating the functions $\mathcal{G}(a, m ; \tau)$ and $\mathcal{G}(a,-m ;-1 / \tau)$ through the measure $d \mu(a)$. This statement is rather hard to check in field theory because there is in general no closed expression for the $\mathcal{G}(a, m ; \tau)$. In appendix B, however, we demonstrate this explicitly in the limit $m=0$ and $b=1$ and explain some of the subtleties involved in making this statement precise.

On the other hand, in the context of the AGT correspondence, it has been checked in $[35,38]$ that the partition function $\mathcal{Z}\left(a, a^{\prime}, m\right)$ on a squashed three-sphere of the $\mathcal{T}(\mathrm{SU}(2))$ theory is precisely equal to the S-duality kernel of a normalized conformal block in Liouville 
theory [43] under the relevant identification of parameters. We are not aware of a similar computation for $N>2$ and Toda theory.

\subsection{Supersymmetric loop operators}

Since we can embed the mass-deformed $\mathcal{T}(\mathrm{SU}(N))$ theory as a domain wall in the four-dimensional $\mathcal{N}=2^{*}$ theory on an ellipsoid, it is natural to think that supersymmetric loop operators in the two theories on $\{x<0\}$ and $\{x>0\}$ are related. In particular, one can introduce a loop operator on one hemisphere and push it through the domain wall to find another loop operator on the other hemisphere. For an S-duality wall one expects that this process turns a Wilson loop operator in the four-dimensional $\mathcal{N}=2^{*}$ theory into a 't Hooft loop operator.

Let us briefly summarize a few facts that are known about supersymmetric loop operators on the four-sphere. The four-sphere partition function can for instance be enriched with Wilson and 't Hooft loop operators. To preserve half of the supersymmetries such loop operators should be supported on the circle

$$
\begin{aligned}
& x_{0}=\cos \rho \\
& x_{1}=b \sin \rho \cos \varphi \\
& x_{2}=b \sin \rho \sin \varphi \\
& x_{3}=x_{4}=0,
\end{aligned}
$$

where $0<\varphi<2 \pi$ and $0<\rho<\pi$, or alternatively supported on the circle obtained by interchanging $b \leftrightarrow b^{-1}$ and $\left\{x_{1}, x_{2}\right\} \leftrightarrow\left\{x_{3}, x_{4}\right\}$. The support of the loop operator lies in the squashed three-sphere at the equator $\left\{x_{0}=0\right\}$ when $\rho=\pi / 2$. However, the expectation value of the loop operator is independent of $\rho$.

Wilson loops. Supersymmetric Wilson loops in the four-dimensional $\mathcal{N}=2^{*}$ theory are labeled by irreducible representations of the gauge group $G$. The expectation values of supersymmetric Wilson loops on the ellipsoid have been computed in [39].

The expectation value for a supersymmetric Wilson loop in the irreducible representation $R$ around a circle in the $\left(x_{1}, x_{2}\right)$-plane is obtained by inserting the factor

$$
W_{R}(a)=\sum_{w \in R} e^{-2 \pi b(w, a)}
$$

into the matrix integral. For example, for a rank $r$ antisymmetric tensor representation of $\mathrm{SU}(N)$ we insert the factor

$$
W_{\left(1^{r}\right)}(a)=\sum_{\left\{j_{1}<\ldots<j_{r}\right\}} e^{-2 \pi b\left(a_{j_{1}}+\cdots+a_{j_{r}}\right)} .
$$

The expectation value for supersymmetric Wilson loops in the $\left(x_{3}, x_{4}\right)$-plane is obtained by replacing $b \rightarrow b^{-1}$.

Sometimes it is convenient to normalize the above expression by dividing by the quantum dimension $\operatorname{dim}_{q} R$ of the representation, where $q=e^{i \pi b^{2}}$, but we will not do this here. 
't Hooft loops. A supersymmetric 't Hooft loop is defined by computing the path integral in the presence of a singular boundary condition along a circle that preserves half of the supersymmetries. The boundary condition is specified by the image of an abelian 't Hooft monopole under a homomorphism $\rho: \mathrm{U}(1) \rightarrow G$, with gauge transformations acting by conjugation on $\rho$. These configurations are classified by irreducible representations $R$ of the Langlands dual ${ }^{L} G$ [44].

The expectation values of supersymmetric 't Hooft loop operators in the $\mathcal{N}=2^{*}$ theory on the round four-sphere have been computed in [27]. It was found that the expectation value can be expressed as (where now $b=1$ )

$$
\int d a \overline{\mathcal{Z}(a, m ; \tau)}\left(T_{R} \cdot \mathcal{Z}(a, m ; \tau)\right)
$$

where $T_{R}$ is a difference operator that acts on the Coulomb branch parameters $a$. The difference operator takes the general form

$$
T_{R} \cdot \mathcal{Z}(a)=\sum_{\nu} C_{\nu}(a, m) \mathcal{Z}(a+i \nu),
$$

where the sum is taken over the weights $\nu$ of the representation $R$.

For the antisymmetric tensor representations $R=\left(1^{r}\right)$ the coefficients $C_{\nu}(a, m)$ only receive one-loop contributions. In this case

$$
C_{\nu}(a, m)=\prod_{\substack{j \in I \\ k \notin I}}\left[\frac{\sinh \pi\left(a_{j k}-m\right) \sinh \pi\left(-a_{j k}-m\right)}{\sinh \pi\left(a_{j k}\right) \sinh \pi\left(-a_{j k}\right)}\right]^{1 / 2},
$$

where we have denoted the weights of the $r$-th antisymmetric tensor representation by $\nu=\sum_{j \in I} h_{j}$ for $I=\left\{j_{1}<\ldots<j_{r}\right\}$. For general representations $R$ there are additional non-perturbative monopole bubbling contributions to the coefficients $C_{\nu}(a, m)$.

Here, we want to re-express the expectation value of the 't Hooft operator in terms of a difference operator $\widetilde{T}_{R}$ acting on the half-sphere partition function $\mathcal{G}(a, m ; \tau)$ in the case $b=1$. In other words, the expectation value of the 't Hooft loop is given by

$$
\int d a \mu(a) \overline{\mathcal{G}(a, m ; \tau)}\left(\widetilde{T}_{R} \cdot \mathcal{G}(a, m ; \tau)\right) \text {. }
$$

The difference operator $\widetilde{T}_{R}$ is related to $T_{R}$ by conjugating with the one-loop factor that relates the Nekrasov partition function $\mathcal{Z}(a, m ; \tau)$ to the half-sphere partition function $\mathcal{G}(a, m ; \tau)$. Later it will be important that $\widetilde{T}_{R}$ is self-adjoint with respect to the measure $\mu(a) d a$.

In appendix $\mathrm{C}$, we perform this conjugation explicitly for the antisymmetric tensor representations to find

$$
\widetilde{T}_{\left(1^{r}\right)} \cdot \mathcal{G}(a)=\sum_{|I|=r} \prod_{\substack{j \in I \\ k \notin I}} \frac{\sin \pi\left(-i a_{j k}-i m\right)}{\sin \pi\left(i a_{j k}\right)} \mathcal{G}\left(a+i \sum_{j \in I} h_{j}\right) .
$$

Remarkably, this difference operator is in agreement with the difference operators $G_{\left(1^{r}\right)}^{(3 \mathrm{~d})}(=$ $\left.\tilde{G}_{\left(1^{r}\right)}^{(3 \mathrm{~d})}\right)$ that introduce codimension-two defects in the $\mathcal{T}(\mathrm{SU}(N))$ theory by acting on the three-dimensional partition function $\mathcal{Z}\left(a, a^{\prime}, m\right)$, in the limit $b \rightarrow 1$. 


\subsection{Intertwining Wilson and 't Hooft loops}

Let us now explain why the difference operators $G_{R}^{(3 \mathrm{~d})}$ are related to 't Hooft operators $\widetilde{T}$. We consider the four-sphere partition function in the presence of both an S-duality wall and a supersymmetric loop operator. Recall that on the lower half-sphere $\left\{x_{0}<0\right\}$ we have the gauge group $\mathrm{SU}(N)$ for which the Wilson loops are labelled by irreducible representations of $\mathrm{SU}(N)$. On the upper half-sphere $\left\{x_{0}>0\right\}$, we have the gauge group ${ }^{L} \mathrm{SU}(N)$ for which the 't Hooft operators are labelled by irreducible representations of $\mathrm{SU}(N)$.

Thus, let us now consider an 't Hooft loop labelled by an irreducible representation of $\mathrm{SU}(N)$ inserted at some point $\rho>\pi / 2$ in the upper half-sphere $\left\{x_{0}>0\right\}$. The expectation value of this system takes the form

$$
\int d a d a^{\prime} \mu(a) \mu\left(a^{\prime}\right) \overline{\mathcal{G}(a, m ; \tau)} \mathcal{Z}\left(a, a^{\prime}, m\right)\left[\widetilde{T}_{\left(1^{r}\right)}\left(a^{\prime}\right) \cdot \mathcal{G}\left(a^{\prime}, m ; \tau\right)\right],
$$

where for simplicity we focus on antisymmetric tensor representations.

Now, the expectation value is independent of the position $\rho$. Thus we can imagine moving the 't Hooft loop through the S-duality domain wall to some point $\rho<\pi / 2$ in the region $\left\{x_{0}<0\right\}$. According to the transformation of loop operators under S-duality, it should become a Wilson loop in the antisymmetric tensor representation $R=\left(1^{r}\right)$. At the level of the partition function, since the operator $\widetilde{T}_{\left(1^{r}\right)}\left(a^{\prime}\right)$ is self-adjoint with respect to the measure $\mu\left(a^{\prime}\right)$, we can bring it to act on $\mathcal{Z}\left(a, a^{\prime}, m\right)$. Provided that $\mathcal{Z}\left(a, a^{\prime}, m\right)$ is an eigenfunction such that

$$
\widetilde{T}_{\left(1^{r}\right)}\left(a^{\prime}\right) \cdot \mathcal{Z}\left(a, a^{\prime}, m\right)=W_{\left(1^{r}\right)}(a) \mathcal{Z}\left(a, a^{\prime}, m\right),
$$

we find

$$
\begin{aligned}
\int d a d a^{\prime} & \mu(a) \mu\left(a^{\prime}\right) \overline{\mathcal{G}(a, m ; \tau)} \mathcal{Z}\left(a, a^{\prime}, m\right)\left[\widetilde{T}_{\left(1^{r}\right)}\left(a^{\prime}\right) \cdot \mathcal{G}\left(a^{\prime}, m ; \tau\right)\right] \\
& =\int d a d a^{\prime} \mu(a) \mu\left(a^{\prime}\right) \overline{\mathcal{G}(a, m ; \tau)}\left[\widetilde{T}_{\left(1^{r}\right)}\left(a^{\prime}\right) \cdot \mathcal{Z}\left(a, a^{\prime}, m\right)\right] \mathcal{G}\left(a^{\prime}, m ; \tau\right) \\
& =\int d a d a^{\prime} \mu(a) \mu\left(a^{\prime}\right)\left[\overline{W_{\left(1^{r}\right)}(a) \mathcal{G}(a, m ; \tau)}\right] \mathcal{Z}\left(a, a^{\prime}, m\right) \mathcal{G}\left(a^{\prime}, m ; \tau\right)
\end{aligned}
$$

which is the expectation value of an S-duality domain wall together with a Wilson loop in the representation $\left(1^{r}\right)$ at some point $\rho<\pi / 2$. Thus compatibility with S-duality demands that $\mathcal{Z}\left(a, a^{\prime}, m\right)$ intertwines 't Hooft loops and Wilson loops according to equation (5.17). See figure 10. In section 4 we have argued that three-dimensional mirror symmetry requires $\mathcal{Z}\left(a, a^{\prime}, m\right)$ to obey the same relation with respect to the three-dimensional limit of the surface defect operators $G_{\left(1^{r}\right)}^{(3 \mathrm{~d})}$. Thus the corresponding operators should agree. Above we checked that this is indeed the case for a round four-sphere.

Let us now make some comments on non-minuscule representations $R$. Since Wilson loop operators labeled by $R$ are defined by a trace over the representation $R$, they obey the character algebra

$$
W_{R_{1}} \circ W_{R_{2}}=\sum_{R_{3}} N_{R_{1}, R_{2}}^{R_{3}} W_{R_{3}}
$$




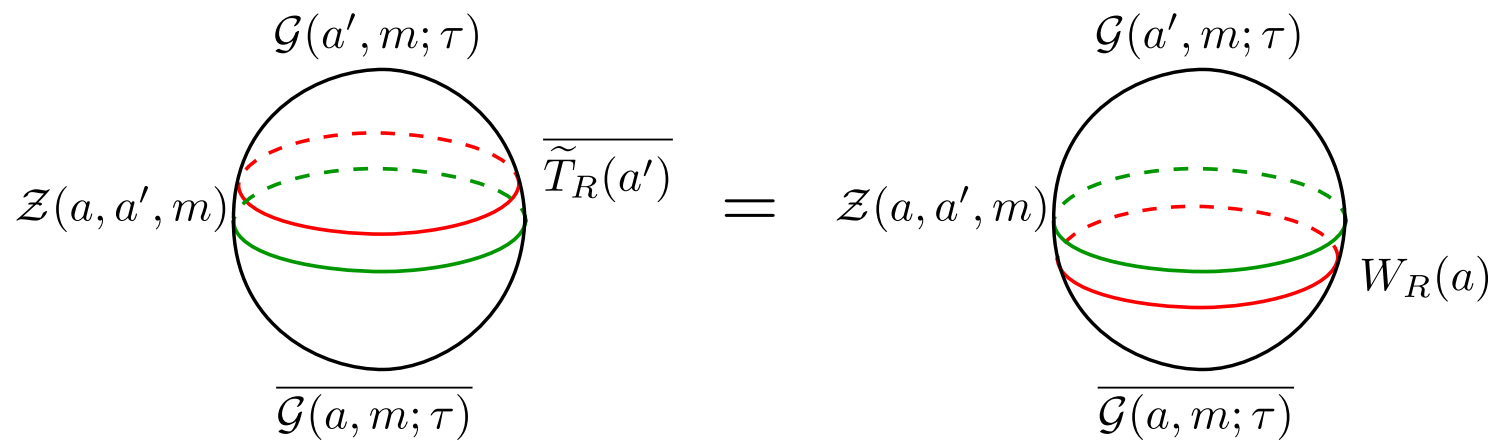

Figure 10. A 't Hooft loop operator $T_{R}$ can be moved through the S-duality domain wall to obtain a Wilson loop operator $W_{R}$.

where $N_{R_{1}, R_{2}}{ }^{R_{3}}$ are the standard Littlewood-Richardson coefficients. In particular, they can be generated from Wilson loops labeled by fully antisymmetric tensor representations by composition and addition/subtraction.

Therefore, we can define a new set of operators $\hat{T}_{R}$ by taking $\hat{T}_{\left(1^{r}\right)} \equiv \widetilde{T}_{\left(1^{r}\right)}$, or equivalently $\hat{T}_{\left(1^{r}\right)} \equiv G_{\left(1^{r}\right)}^{(3 \mathrm{~d})}$, for antisymmetric representations and imposing the character algebra

$$
\hat{T}_{R_{1}} \circ \hat{T}_{R_{2}}=\sum_{R_{3}} N_{R_{1}, R_{2}}^{R_{3}} \hat{T}_{R_{3}}
$$

The resulting operators $\hat{T}_{R}$ automatically transform in the expected way under S-duality, and it is natural to expect that these operators encode the expectation value of 't Hooft loops for general representations.

However, we emphasize that the $\hat{T}_{R}$ do not seem to correspond to the expectation value of a 't Hooft loop with magnetic weight given by the highest weight of the representation $R$, when the representation is non-minuscule. For example, for $\mathrm{SU}(2)$ the 't Hooft loop whose magnetic weight is double that of the 't Hooft loop of minimal charge is given by $T_{1} \circ T_{1}$ rather than $T_{1} \circ T_{1}-T_{0}$. This is again an invertible linear transformation on the algebra of the operators. In this case, the origin of the basis transformation is a natural resolution of the Bogomolnyi moduli space that arises for representations with nonperturbative monopole bubbling effects [27]. Once again, we emphasize that the simplest and unambiguous operators are those in antisymmetric tensor representations.

\subsection{Verlinde operators in Toda CFT}

All we have discussed so far in this section can also be framed in the language of Liouville or Toda conformal field theory. This approach has the benefit that, at least for the 't Hooft loop in the fundamental representation, we can compute the required operator for general squashing parameter $b$.

Let us briefly review aspects of this correspondence. For the $\mathcal{N}=2^{*}$ theory with gauge group $\mathrm{SU}(N)$, the ellipsoid partition function is related to a Liouville or type $A_{N-1}$ Toda correlator on the punctured torus with an insertion of a semi-degenerate primary field. The parameters on both sides of the correspondence are related as follows: 


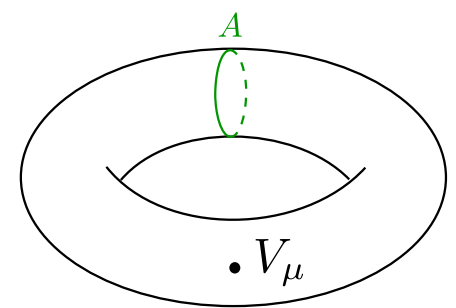

Figure 11. The four-sphere partition function of the $\mathcal{N}=2^{*}$ theory is equal to a Toda correlation function on the punctured torus with a semi-degenerate vertex operator $V_{\mu}$, with momentum $\mu$, inserted at the puncture.

1. The geometric parameter $b$ is a dimensionless coupling in the conformal field theory and gives the central charge $c=(N-1)\left(1+N(N+1) q^{2}\right)$, where $q=b+b^{-1}$.

2. The holomorphic gauge coupling $\tau$ is the complex structure parameter of the punctured torus.

3. The mass $m$ of the adjoint hypermultiplet is encoded in the momentum of the semidegenerate primary field,

$$
\mu=N\left(\frac{q}{2}+i m\right) \omega_{N-1}
$$

Choosing a pants decomposition, the correlation function of the primary field on the punctured torus can be written as an expansion in Liouville or $W_{N}$-algebra conformal blocks

$$
\int d \alpha C(\mu, \alpha, 2 Q-\alpha) \overline{\mathcal{F}(\alpha, \mu ; \tau)} \mathcal{F}(\alpha, \mu ; \tau)
$$

where the integral is over non-degenerate momenta $\alpha=Q+i a$, with $a \in \mathbb{R}^{N-1}$ and $Q=q \rho$, where $\rho$ is the Weyl vector of $A_{N-1}$.

The conformal blocks $\mathcal{F}(a, \mu ; \tau)$ are normalized to contain the classical and instanton contributions to the Nekrasov partition function. The three-point function $C(\mu, \alpha, 2 Q-\alpha)$ is proportional (up to an $m$-dependent piece that can be absorbed in the normalization of the primary field) to the modulus squared of the 1-loop contribution $\left|\mathcal{G}_{1-\text { loop }}\right|^{2}$ times the measure $\mu(a)$. The meromorphic function $\mathcal{G}(a, m ; \tau)$ that we introduced earlier corresponds to a convenient normalization of the conformal block $\mathcal{F}(\alpha, \mu ; \tau)$ that absorbs the three-point functions. This is an extension to higher rank of a frequently used normalization in Liouville theory [28].

Loop operators in the four-dimensional gauge theory are realized as Verlinde operators in the dual conformal field theory [9, 28]. The Verlinde operators act on the space of Virasoro or $W_{N}$-algebra conformal blocks by transporting a chiral primary field around a simple closed curve $C$ on the Riemann surface. The operators constructed in this way depend only on the homotopy class of the curve $C$ up to a choice of 'framing' that will not be important here.

If we choose the pants decomposition of the punctured torus determined by the A-cycle in figure 11, a supersymmetric Wilson loop in $\mathcal{N}=2^{*}$ theory in the rank $r$ antisymmetric 
tensor representation corresponds to transporting a degenerate chiral primary with momentum $\eta=-b \omega_{j}$ around that A-cycle. The resulting expression changes from the original conformal block by the factor

$$
W_{\left(1^{r}\right)}^{\mathrm{CFT}}=\sum_{\left\{j_{1}<\ldots<j_{r}\right\}} e^{-2 \pi b\left(a_{j_{1}}+\cdots+j_{r}\right)},
$$

which is in agreement with the localization computation.

An 't Hooft loop in the $r$-th fundamental representation corresponds to transporting the same chiral primary around the B-cycle of the punctured torus. This Verlinde operator has been computed directly in Toda theory for the fundamental representation in [30]. Acting on the conformal blocks $\mathcal{F}(\alpha, \mu ; \tau)$, the operator is given by

$$
T_{(1)}^{\mathrm{CFT}} \cdot \mathcal{F}(\alpha)=\sum_{j=1}^{N} \prod_{k \neq j}^{N} \frac{\Gamma\left(i b a_{j k}\right) \Gamma\left(b q+i b a_{j k}\right)}{\Gamma\left(\frac{b q}{2}+i b a_{j k}-i b m\right) \Gamma\left(\frac{b q}{2}+i b a_{j k}+i b m\right)} \mathcal{F}\left(\alpha-b h_{j}\right),
$$

where $\alpha=Q+i a$ is the momentum around the loop that defines the pants decomposition.

To construct an operator that acts on the normalized conformal blocks $\mathcal{G}(a, m ; \tau)$, we have to conjugate by the one-loop contribution (5.6). In appendix $\mathrm{C}$ we perform this conjugation to find

$$
\widetilde{T}_{(1)}^{\mathrm{CFT}} \cdot \mathcal{G}(a)=\sum_{j=1}^{N} \prod_{k \neq j}^{N} \frac{\sin \pi b\left(\frac{q}{2}-i a_{j k}-i m\right)}{\sin \pi b\left(-i a_{j k}\right)} \mathcal{G}\left(a+i b h_{j}\right),
$$

which is precisely equal to the three-dimensional operator $G_{(1)}^{(3 \mathrm{~d})}$ for any real $b$ (see equation (4.10)). This provides another check on the relation of the difference operators $G_{R}^{(3 \mathrm{~d})}$ to the 't Hooft loop operators for the fundamental representation.

\section{Discussion}

In this paper we generated an algebra of difference operators $G_{R}$ acting on the $\mathcal{N}=2$ superconformal index, labeled by irreducible representations $R$ of $\mathrm{SU}(N)$. Generalizing the arguments of [10], we claim that these difference operators represent half-BPS surface defects in four-dimensional $\mathcal{N}=2$ theories of class $\mathcal{S}$. We discussed several arguments in favour of this claim. Most importantly, we emphasized that it is highly non-trivial that we indeed managed to consistently close the algebra, and that the difference operators have a natural interpretation in various dual frames. Let us mention a few open questions and interesting links.

A microscopic gauge theory understanding of these defects is unfortunately still lacking, either in terms of a defect description or alternatively as a description of the twodimensional degrees of freedom living on the support of the defect. We did find a twodimensional field theory description in two extreme cases: fully antisymmetric and fully symmetric representations. It is however not at all clear that there exists a Lagrangian description for the two-dimensional degrees of freedom living on the support of the surface defect for a generic representation $R$. 


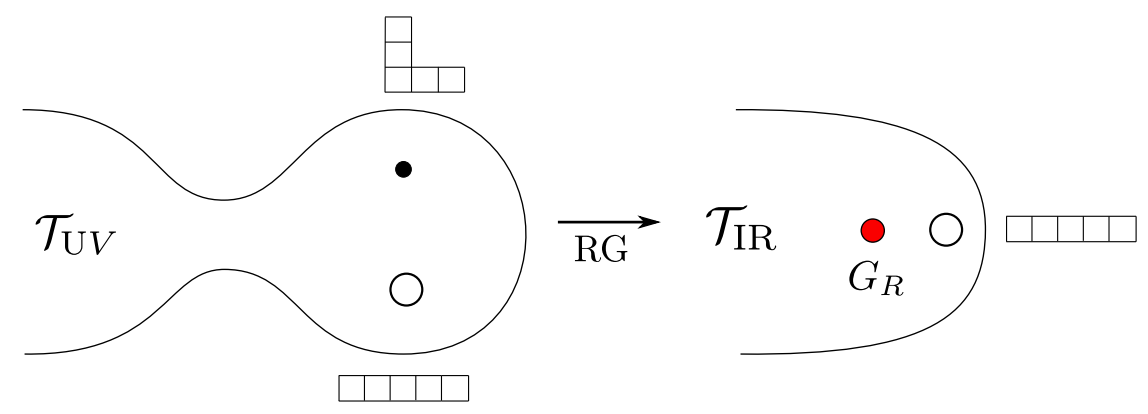

Figure 12. Left: UV curve of the theory $\mathcal{T}_{\mathrm{UV}}$, obtained by gluing a three-punctured sphere with two full punctures and one hook-shaped puncture to the UV curve of the theory $\mathcal{T}_{\text {IR }}$. Right: a renormalization group flow connects $\mathcal{T}_{\mathrm{UV}}$ to $\mathcal{T}_{\mathrm{IR}}$ with a possibly general surface defect $G_{R}$.

The operators $G_{R}$ can be written as a sum of weights in the representation $R$, which in the field theory description of the defect should have the interpretation as a sum over vacua. In case a weight $\lambda$ appears with multiplicity one in the representation $R$, the contribution to $G_{R}$ is a single ratio of theta-function and seems likely to have an interpretation as the contribution to the superconformal index in a vacuum characterized by $\lambda$. When the weight $\lambda$ appears with higher multiplicity, however, the contribution to $G_{R}$ is a sum of such ratios and is less likely to have such an interpretation.

A similar structure can be observed from the perspective of the AGT correspondence. In particular, reference [31] has demonstrated that a ratio of Toda correlation functions involving a degenerate momentum $\mu=-b h_{1}$ captures the two-sphere partition function of the $\mathcal{N}=(2,2)$ theory that we have associated to the surface defect labelled by the fundamental representation. However, for representations with multiple weight contributions, the Toda three-point function with degenerate insertions (see [45, 46]) do not appear to have the structure of one-loop contributions to the two-sphere partition function of an $\mathcal{N}=(2,2)$ theory.

As briefly mentioned in the introduction and main text, we expect that there is an alternative method to find the difference operators $G_{R}$. Instead of coupling the theory $\mathcal{T}_{\text {IR }}$ to a bifundamental hypermultiplet, corresponding to adding a puncture with $\mathrm{U}(1)$ symmetry, one could add a puncture with a larger flavor symmetry group. This generically involves coupling $\mathcal{T}_{I R}$ to a non-Lagrangian theory corresponding to a sphere with two full punctures and one intermediate puncture. An example is illustrated in figure 12. The superconformal index for $\mathcal{T}_{\mathrm{UV}}$ should then contain a larger spectrum of residues. One might expect any difference operator $G_{R}$ to originate from such a residue computation.

Again, this is analogous to the Toda perspective, where non-maximal flavor punctures correspond to insertions of semi-degenerate vertex operators. By analytic continuation correlation functions of such operators have poles, whose residues correspond to reductions to a completely degenerate vertex operator. If we insert a semi-degenerate vertex operator that corresponds to a simple $\mathrm{U}(1)$ puncture, we can only access completely degenerate vertex operators with momentum $\alpha=-b \lambda_{1}-b^{-1} \lambda_{2}$, where $\lambda_{1}=r_{1} \omega_{1}$ and $\lambda_{2}=r_{2} \omega_{2}$ are the highest weights of two symmetric tensor representations. To find completely degenerate 
vertex operators with generic weights $\lambda_{1}$ and $\lambda_{2}$, one must insert a generic semi-degenerate vertex operator, corresponding to a generic flavor puncture.

The difference operators $G_{R}$ are elliptic generalizations of the Macdonald operators. Although these operators have not been constructed mathematically for all representations $R$ (as far as we know), the elliptic Ruijsenaars-Schneider difference operators have been related to exterior powers of the vector representation of the elliptic quantum group $E_{\tau, \eta}\left(g l_{N}\right)$ [47]. It would be very interesting to interpret this connection to elliptic quantum groups physically. This relation could appear naturally when interpreting the difference operators $G_{R}$ in terms of a three-dimensional topological field theory on $C \times S^{1}$.

In the Macdonald limit $p=0$ we have found that the difference operators correspond to Wilson loops in an analytic continuation of refined Chern-Simons theory on $C \times S^{1}$ (see section 3). The particular ratio of modular S-matrices that appears in the operator product expansion of the $G_{R}$, suggests that when we take a Wilson loop operator close to a puncture on $C$, it can be interpreted as a Verlinde loop operator on the boundary torus. For example, taking the Schur limit $t=q$, and replacing $q \rightarrow \exp (2 \pi i /(k+N))$ in the modular S-matrix, we would recover the modular S-matrix elements for characters of integrable representations of the affine current algebra $\widehat{\mathfrak{s u}}(N)_{k}$. However, for the superconformal index it is important that we have an analytic continuation of this statement to $|q|<1$.

In the dimensional reduction of the four-dimensional superconformal index to a threedimensional partition function, we found that the difference operators $G_{R}$ are related to operators that introduce line defects. For theories of class $\mathcal{S}$, there is a mirror description as a star-shaped quiver and we showed that the difference operators introduce Wilson loops for the central node of the quiver, at least in the case of antisymmetric tensor representations. For non-minuscule representations, we found that there is some mixing.

It would be interesting and important to understand these line defects in threedimensions from first principles by localization. For the fully symmetric and anti-symmetric tensor representations, we expect this could be done by coupling to a supersymmetric quantum mechanics on a circle, in a similar spirit to [21] but in one dimension lower. On the other hand, we expect that the rank- $r$ anti-symmetric tensor operator has another description as a monodromy defect breaking the gauge group to $S(\mathrm{U}(r) \times \mathrm{U}(N-r)$ ), which might also be used to perform an exact localization computation by extending the computations of $[48,49]$ for abelian monodromy defects.

\section{Acknowledgments}

We would very much like to thank Fernando Alday for collaboration on part of this project. The work of M.B. is supported by the Perimeter Institute for Theoretical Physics. Research at the Perimeter Institute is supported by the Government of Canada through Industry Canada and by the Province of Ontario through the Ministry of Research and Innovation. The work of M.F. and P.R. is supported by ERC STG grant 306260. The work of L.H. is supported by a Royal Society Dorothy Hodgkin fellowship. 


\section{A Macdonald polynomials and the refined S-matrix}

\section{A.1 Group theory}

The finite dimensional irreducible representations of $A_{N}$ are in one-to-one correspondence with dominant integral weights,

$$
\lambda=\sum_{i=1}^{N-1} \lambda_{i} \omega_{i}
$$

whose Dynkin labels $\left(\lambda_{1}, \lambda_{2}, \ldots, \lambda_{N-1}\right)$ are nonnegative integers. Equivalently, irreducible representations are labeled by partitions $\left(\ell_{1}, \ell_{2}, \ldots, \ell_{N}\right)$ where $\ell_{1} \geq \ell_{2} \geq \ldots \geq \ell_{N}=0$, such that

$$
\ell_{i}=\lambda_{i}+\lambda_{i+1}+\ldots+\lambda_{N-1}
$$

Each partition is associated to a Young diagram whose $i$-th row has length $\ell_{i}$. For instance, the following diagram

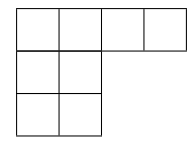

corresponds to the partition $(4,2,2,0)$. The partition labels $\left(\ell_{1}, \ell_{2}, \ldots, \ell_{N}\right)$ are related to the components of the weight in the orthogonal basis

$$
\omega_{i}=\epsilon_{1}+\cdots+\epsilon_{i}-\frac{i}{N} \sum_{j=1}^{N} \epsilon_{j}
$$

where

$$
\lambda=\sum_{i=1}^{N} \kappa_{i} \epsilon_{i}, \quad \kappa_{i}=\ell_{i}-\frac{1}{N} \sum_{j=1}^{N-1} j\left(\ell_{j}-\ell_{j+1}\right) .
$$

The states in a given irreducible representation are in one-to-one correspondence with semi-standard Young tableaux. They are obtained by filling the boxes of a Young diagram with the numbers $\{1, \ldots, N\}$, such that the numbers are non-decreasing from left to right and strictly increasing from top to bottom. Finally, to each semi-standard Young tableau, we attach the labels $\left(n_{1}, \ldots, n_{N}\right)$, where $n_{i}$ denotes the number of times that $i$ appears in the semi-standard tableau. As an example below we include a few semi-standard tableaux for the adjoint 8 representation of $\mathrm{SU}(3)$ with their corresponding labels.

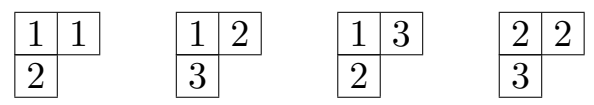

$$
\begin{aligned}
& (2,1,0) \quad(1,1,1) \quad(1,1,1) \quad(0,2,1)
\end{aligned}
$$

\section{A.2 Schur polynomials and the modular S-matrix}

Introduce coordinates $a_{j}$, for $j=1, \ldots, N$, obeying $\prod_{i=1}^{N} a_{i}=1$. For $a_{j}=e^{i \theta_{j}}$ they are coordinates on the maximal torus of $\mathrm{SU}(N)$. The Schur polynomials form a basis of symmetric functions in the variables $\left\{a_{1}, \ldots, a_{N}\right\}$ labeled by irreducible representations. The 
Schur polynomial of the irreducible representation $\lambda$ labeled by the partition $\left(\ell_{1}, \ldots, \ell_{N}\right)$ is given by the determinant formula

$$
\chi_{\lambda}(a)=\frac{\operatorname{det} a_{j}^{\ell_{i}+N-i}}{\operatorname{det} a_{j}^{N-i}} .
$$

An important property of the Schur polynomials is that they are orthonormal with respect to the inner product on the space of symmetric functions

$$
\langle f, g\rangle=\int \Delta(a) f(a) g\left(a^{-1}\right)
$$

where

$$
\Delta(a)=\frac{1}{N !} \prod_{i=1}^{N-1} \frac{d a_{i}}{2 \pi i a_{i}} \prod_{i \neq j}\left(1-\frac{a_{i}}{a_{j}}\right) .
$$

is the Haar measure and the integration is over the maximal torus of $\mathrm{SU}(N)$. Products of Schur polynomials decompose according to the tensor product of the irreducible representations

$$
\chi_{\lambda_{1}}(a) \chi_{\lambda_{1}}(a)=\sum_{\mu} N_{\lambda_{1}, \lambda_{2}}{ }^{\mu} \chi_{\mu}(a)
$$

where $N_{\lambda_{1}, \lambda_{2}}{ }^{\mu}$ are the Littlewood-Richardson numbers.

In order to construct the modular S-matrix we introduce the Weyl weight $\rho$, which is the highest weight of the adjoint representation of $\mathrm{SU}(N)$. Its components in the Dynkin basis are $\rho=(1,1, \ldots, 1)$. In the orthogonal basis mentioned above,

$$
\rho=\left(\frac{N-1}{2}, \frac{N-3}{2}, \ldots, \frac{1-N}{2}\right)
$$

and we will denote these components by $\rho_{j}=(N-2 j+1) / 2$. Now consider two irreducible representations $\lambda$ and $\lambda^{\prime}$ with components $\kappa_{i}$ and $\kappa_{i}^{\prime}$ in the orthogonal basis. Then the modular S-matrix is given by

$$
S_{\lambda \lambda^{\prime}}=S_{00} \chi_{\lambda}\left(q^{\rho_{1}}, \ldots, q^{\rho_{N}}\right) \chi_{\bar{\lambda}^{\prime}}\left(q^{\rho_{1}+\kappa_{1}}, \ldots, q^{\rho_{N}+\kappa_{N}}\right),
$$

where $\bar{\lambda}^{\prime}$ denotes the complex conjugate representation of $\lambda^{\prime}$. We will not need the overall normalization $S_{00}$.

\section{A.3 Macdonald polynomials and the refined S-matrix}

The Macdonald polynomials are symmetric polynomials in the variables $\left\{a_{1}, \ldots, a_{N}\right\}$ that depend on two additional complex parameters $q$ and $t$. The Macdonald polynomials are labeled by irreducible representations $\lambda$ of $\mathrm{SU}(N)$ and reduce to the corresponding Schur polynomials when $q=t$.

The Macdonald polynomial labeled by the irreducible representation $\lambda$ is

$$
P_{\lambda}(a, q, t)=\chi_{\lambda}(a)+\sum_{\mu<\lambda} c_{\lambda, \mu}(q, t) \chi_{\mu}(a)
$$


where $c_{\lambda, \mu}(q, t)$ are rational functions of $q$ and $t$ that are uniquely determined by ensuring $P_{\lambda}(a, q, t)$ is a simultaneous eigenfunctions of the difference operators

$$
G_{r}=t^{r(1-N)} \sum_{\substack{I \subset\{1, \ldots, N\} \\|I|=r}} \prod_{i \in I, j \notin I} \frac{t a_{i}-a_{j}}{a_{i}-a_{j}} T_{I}, \quad r=1, \ldots, N-1
$$

where

$$
T_{I}: a_{i} \rightarrow \begin{cases}q^{1-1 / N} a_{i} & \text { if } i \in I \\ q^{-1 / N} a_{i} & \text { if } i \notin I .\end{cases}
$$

Here we have included a background shift by $q^{-1 / N}$ compared to the standard Macdonald difference operators in order to preserve the condition $\prod_{i}^{N} a_{i}=1$ relevant for $\mathrm{SU}(N)$. For example, the first few Macdonald polynomials for $\mathrm{SU}(2)$ are

$$
\begin{aligned}
& P_{0}(a, q, t)=1 \\
& P_{1}(a, q, t)=\chi_{1}(a) \\
& P_{2}(a, q, t)=\chi_{2}(a)+\frac{q-t}{1-q t} \\
& P_{3}(a, q, t)=\chi_{3}(a)+\frac{(q-t)(1+q)}{1-t q^{2}} \chi_{1}(a) \\
& P_{4}(a, q, t)=\chi_{4}(a)+\frac{(q-t)\left(1-q^{3}\right)}{(1-q)\left(1-q^{3} t\right)} \chi_{2}(a)+\frac{q(q-t)\left(1+q^{2}\right)(1-t)}{\left(1-q^{2} t\right)\left(1-q^{3} t\right)} .
\end{aligned}
$$

The difference operators are self-adjoint with respect to the inner product

$$
\langle f, g\rangle=\int \Delta_{q, t}(a) f(a) g\left(a^{-1}\right), \quad \Delta_{q, t}(a)=\frac{1}{N !} \prod_{i=1}^{N-1} \frac{d a_{i}}{2 \pi i a_{i}} \prod_{i \neq j} \frac{\left(a_{i} / a_{j} ; q\right)}{\left(t a_{i} / a_{j} ; q\right)}
$$

where $(a ; q)=\prod_{i=0}^{\infty}\left(1-q^{i} a\right)$ is the q-Pochhammer symbol, and consequently, the Macdonald polynomials are non-degenerate and orthogonal with respect to the same measure. In order to obtain functions orthonormal with respect to the measure, a normalization factor must be included.

The product of Macdonald polynomials decomposes according to the tensor product of irreducible representations

$$
P_{\lambda_{1}}(a, q, t) P_{\lambda_{2}}(a, q, t)=\sum_{\mu} N_{\lambda_{1}, \lambda_{2}}{ }^{\mu}(q, t) P_{\mu}(a, q, t)
$$

where the $N_{\lambda_{1}, \lambda_{2}}{ }^{\mu}(q, t)$ are rational functions in $q$ and $t$.

Analogous to the modular S-matrix, the refined S-matrix is given by

$$
S_{\lambda \lambda^{\prime}}=S_{00} P_{\lambda}\left(t^{\rho_{1}}, \ldots, t^{\rho_{N}}\right) P_{\bar{\lambda}^{\prime}}\left(t^{\rho_{1}} q^{\kappa_{1}}, \ldots, t^{\rho_{N}} q^{\kappa_{N}}\right) .
$$

It is then an easy exercise to check that the ratios $S_{R, S} / S_{0, S}$ are indeed the eigenvalues of the difference operators $G_{R}$ in the Macdonald limit, namely

$$
G_{R} \cdot P_{S}\left(a_{i}, q, t\right)=\frac{S_{R, S}}{S_{0, S}} P_{S}\left(a_{i}, q, t\right) .
$$




\section{B S-duality kernel}

Instead of merely inserting a three-dimensional $\mathcal{N}=2$ vectormultiplet on the threedimensional boundary $\left\{x_{0}=0\right\}$, we could also glue in the three-dimensional mass-deformed linear quiver theory $\mathcal{T}(\mathrm{SU}(N))$. In fact, its $\mathcal{N}=4$ variant was introduced as an S-dual of the Dirichlet boundary condition in the four-dimensional $\mathcal{N}=4$ theory with gauge group $\mathrm{SU}(N)[34,50]$.

It is thus natural to expect that the mass-deformed $\mathcal{T}(\mathrm{SU}(N))$ theory encodes the field theory degrees of freedom on a so-called S-duality domain wall in the $\mathcal{N}=2^{*}$ theory. Such a domain wall is defined so that the four-dimensional theories on either side are related by the transformation $S:(\tau, m) \rightarrow(-1 / \tau,-m)$. In this appendix we will verify that this is indeed the case if we assume that $\mathcal{G}_{b}$ is the partition function on the half-sphere with Dirichlet boundary conditions.

Before introducing the S-duality domain wall, let us briefly consider the ellipsoid partition function $\mathcal{Z}_{S_{b}^{4}}$ of the $\mathcal{N}=2^{*}$ theory with gauge group $\mathrm{SU}(N)$. The AGT correspondence relates this to a Toda correlator on the once-punctured torus. We thus expect that the ellipsoid partition function transforms as a modular form. More precisely, it should transform as [51]

$$
\mathcal{Z}_{S_{b}^{4}}(-m ;-1 / \tau)=|\tau|^{2 \Delta(m)} \mathcal{Z}_{S_{b}^{4}}(m ; \tau),
$$

with modular weight

$$
\Delta(m)=\frac{N(N-1)}{2}\left(\frac{Q^{2}}{4}+m^{2}\right) .
$$

This modular property of the ellipsoid partition function is guaranteed if the halfsphere partition function $\mathcal{G}_{b}$ transforms as

$$
\mathcal{G}_{b}(-m, a ;-1 / \tau)=(-i \tau)^{\Delta(m)} \int d a^{\prime} \mu_{b}\left(a^{\prime}\right) \mathcal{Z}_{b}\left(a_{i}, a^{\prime}, m\right) \mathcal{G}_{b}\left(m, a^{\prime} ; \tau\right)
$$

where we integrate over a real slice of the Coulomb branch (just like in all matrix integrals in the remainder of this section). The integration kernel $\mathcal{Z}_{b}\left(a, a^{\prime}, m\right)$ must obey two important properties. First, it must obey the symmetry

$$
\mathcal{Z}_{b}\left(a, a^{\prime}, m\right)=\mathcal{Z}_{b}\left(a^{\prime}, a,-m\right) .
$$

Second, it must be a unitary with respect to the measure $\mu_{b}(a) d a$, in the sense that

$$
\int d a \mu_{b}(a) \overline{\mathcal{Z}_{b}\left(a^{\prime}, a, m\right)} \mathcal{Z}_{b}\left(a, a^{\prime \prime},-m\right)=\mu_{b}\left(a^{\prime}\right) \delta\left(a^{\prime}, a^{\prime \prime}\right) .
$$

Now consider the ellipsoid partition function with the insertion of an S-duality domain wall. Assuming that $\mathcal{G}_{b}$ is the half-sphere partition function of the $\mathcal{N}=2^{*}$ theory with Dirichlet boundary conditions, the S-duality partition function on the squashed four-sphere should be given by

$$
\begin{aligned}
\int d a \mu(a) \overline{\mathcal{G}_{b}(m, a ; \tau)} & \mathcal{G}_{b}(-m, a ;-1 / \tau) \\
& =\int d a \mu(a) \int d a^{\prime} \mu\left(a^{\prime}\right) \overline{\mathcal{G}_{b}(m, a ; \tau)} \mathcal{Z}_{b}\left(a, a^{\prime}, m\right) \mathcal{G}_{b}\left(m, a^{\prime} ; \tau\right)
\end{aligned}
$$


Consequently, $\mathcal{Z}_{b}\left(a, a^{\prime}, m\right)$ should encode the gauge degrees of freedom localized on the domain wall. Specifically, we expect that $\mathcal{Z}_{b}\left(a, a^{\prime}, m\right)$ is the partition function of the massdeformed $\mathcal{T}(\mathrm{SU}(N))$ theory on a squashed three-sphere.

In this context, the symmetry $\mathcal{Z}_{b}\left(a, a^{\prime}, m\right)=\mathcal{Z}_{b}\left(a^{\prime}, a,-m\right)$ is equivalent to threedimensional mirror symmetry. The unitary property (B.4) follows because the partition function $\mathcal{Z}_{b}\left(a, a^{\prime}, m\right)$ is an eigenfunction of the self-adjoint operator $G_{R}^{(3 \mathrm{~d})}$ with respect to the measure $\mu_{b}(a) d a$ (see equation (4.11)).

Indeed, let us denote the integral (B.4) by

$$
\mathcal{I}\left(a^{\prime}, a^{\prime \prime}\right)=\int d a \mu_{b}(a) \overline{\mathcal{Z}_{b}\left(a^{\prime}, a, m\right)} \mathcal{Z}_{b}\left(a, a^{\prime \prime},-m\right) .
$$

The self-adjoint operator $G_{R}^{(3 \mathrm{~d})}\left(a^{\prime}\right)$ can act inside this integrand in either direction, which must lead to the same answer. Consequently we find

$$
\left(W_{R}(a)-W_{R}\left(a^{\prime \prime}\right)\right) \mathcal{I}\left(a, a^{\prime \prime}\right)=0
$$

where $W_{R}(a)$ is the expectation value of a Wilson loop in the representation $R$. This implies that the integral vanishes if $a \neq a^{\prime \prime}$ modulo Weyl transformations.

\section{B.1 Example}

Let us check the above transformation properties of the half-sphere partition function $\mathcal{G}_{b}$ on the round four-sphere, when $b=1$, and in the $\mathcal{N}=4$ limit, when $m \rightarrow 0$.

First, we compute the explicit expression for $\mathcal{G}_{b=1}\left(\tau, m, a_{i}\right)$ for gauge group $\mathrm{SU}(N)$. Its one-loop contribution (5.6) simplifies to the formula

$$
\mathcal{G}_{1-\text { loop }}\left(m, a_{i}\right)=\frac{1}{\sqrt{2 \pi}} \prod_{i<j} \frac{\pi a_{i j}}{\sinh \left(\pi a_{i j}\right)},
$$

where $a_{i j}=a_{i}-a_{j}$ with the constraint that $\sum_{i=1}^{N} a_{i}=0$. Its classical contribution times its instanton contribution is given by

$$
\mathcal{G}_{\mathrm{cl}}\left(a_{i} ; \tau\right) \mathcal{G}_{\text {inst }}\left(a_{i} ; \tau\right)=e^{-\pi i \tau\left(\sum_{i=1}^{N} a_{i}^{2}\right)} m(\tau)^{1-N} .
$$

This can be argued as follows. If the gauge group would be $\mathrm{U}(N)$, the instanton contribution would be $\mathcal{G}_{\text {inst }}=1$ [52]. For gauge group $\mathrm{SU}(N)$, however, one must first divide by the $\mathrm{U}(1)$ factor. We can find this $\mathrm{U}(1)$ factor by comparing with the $q=\exp (\tau)$-expansion of the Toda conformal block

$$
\mathcal{F}\left(a_{i} ; \tau\right)=q^{\Delta\left(a_{i}\right)-\frac{c}{24}} \sum_{k} q^{k} F_{k}
$$

In particular, using the known expressions for the Toda central charge $c$ and the momentum $\Delta\left(a_{i}\right)$, we can verify the classical contribution to $\mathcal{G}_{\text {cl }} \mathcal{G}_{\text {inst }}$ for any $N$. Furthermore, we can match the full expressions in an expansion of the instanton parameter $q$ for $N=2,3$. 
Putting the pieces together, we have

$$
\mathcal{G}_{b=1}\left(a_{i} ; \tau\right)=e^{-\pi i \tau\left(\sum_{i=1}^{N} a_{i}^{2}\right)} \frac{1}{\sqrt{2 \pi}} \prod_{i<j} \frac{\pi a_{i j}}{\sinh \left(\pi a_{i j}\right)} m(\tau)^{1-N} .
$$

After performing $(N-1)$ Gaussian integrals we expect to find the partition function

$$
\mathcal{Z}_{S_{b=1}^{4}}(\tau) \sim \frac{1}{|m(\tau)|^{2(N-1)} \operatorname{Im}(\tau)^{\left(N^{2}-1\right) / 2}}
$$

which has the expected transformation

$$
\mathcal{Z}_{S_{b=1}^{4}}\left(-\frac{1}{\tau}\right)=|\tau|^{N(N-1)} \mathcal{Z}_{S_{b=1}^{4}}(\tau)
$$

under S-duality. We have indeed verified this for $N=2,3$. In the above, we have used $\mu_{b=1}(a)=\prod_{i<j} 4 \sinh \left(\pi a_{i j}\right)^{2}$ and $\Delta(0)=\frac{N(N-1)}{2}$.

We can also check that the three-sphere partition function

$$
\mathcal{Z}_{b=1}\left(a_{i}, a_{i}^{\prime}\right)=\frac{\sum_{\rho \in S_{N}}(-1)^{\rho} e^{2 \pi \sum_{j=1}^{N} a_{\rho(j)} a_{j}^{\prime}}}{\prod_{i<j} 2 \sinh \pi\left(a_{i j}\right) 2 \sinh \pi\left(a_{i j}^{\prime}\right)},
$$

is the S-duality kernel for the half-sphere partition function $\mathcal{G}_{b=1}\left(\tau, a_{i}\right)$. This is again a matter of performing Gaussian integrals and using the modular property of the $\eta$-function. In particular, for $N=2,3$ we explicitly verified that

$$
\int d a_{i}^{\prime} \mu_{b=1}\left(a_{i}^{\prime}\right) \mathcal{Z}_{b=1}\left(a_{i}, a_{i}^{\prime}\right) \mathcal{G}_{b=1}\left(a_{i}^{\prime} ; \tau\right) \sim(-i \tau)^{\frac{-N(N-1)}{2}} \mathcal{G}_{b=1}\left(a_{i} ;-1 / \tau\right) .
$$

This completes the argument and gives some evidence that $\mathcal{G}_{b}$ is indeed the half-sphere partition function with Dirichlet boundary conditions.

\section{Factorization of Toda 3-point function}

Let us briefly review some properties of special functions we need in order to manipulate one-loop contributions. As in the main text, $b \in \mathbb{R}_{>0}$ is a real parameter and we define $q \equiv b+b^{-1}$.

The double gamma function $\Gamma_{b}(x)$ is a meromorphic function of $x$ characterized by the functional equation

$$
\Gamma_{b}(x+b)=\sqrt{2 \pi} b^{b x-\frac{1}{2}} \Gamma_{b}(x) / \Gamma(b x)
$$

where $\Gamma(x)$ is the Euler gamma function and its value $\Gamma_{b}(q / 2)=1$. We will also need the double sine function, which is a meromorphic function that can be defined in terms of the double gamma function by the formula $S_{b}(x) \equiv \Gamma_{b}(x) / \Gamma_{b}(q-x)$. The double sine function is characterized by the functional equation

$$
S_{b}(x+b)=2 \sin (\pi b x) S_{b}(x)
$$


We will furthermore need the function $\Upsilon_{b}(x)^{-1}=\Gamma_{b}(x) \Gamma_{b}(q-x)$ which is entire analytic. A more complete discussion of the properties of these functions can be found, for example, in [53].

Let us begin by considering the three-point function $C(\alpha, 2 Q-\alpha, \nu)$ in $A_{N-1}$ Toda theory corresponding to the trivalent vertex in the pants decomposition of a torus with a simple puncture. The momentum in the internal channel $\alpha=Q+i a$, with $a \in \mathbb{R}$, is non-degenerate and describes a delta-function normalizable state, while the momentum $\nu=N(q / 2+i m) \omega_{N-1}$, with $m \in \mathbb{R}$, is semi-degenerate. Substituting these momenta into the more general result of $[45,46]$ we find that

$$
C(\alpha, 2 Q-\alpha, \nu)=f(m) \frac{\prod_{i<j}^{N} \Upsilon_{b}\left(i a_{i j}\right) \Upsilon_{b}\left(-i a_{i j}\right)}{\prod_{i, j=1}^{N} \Upsilon_{b}\left(\frac{q}{2}+i a_{i j}+i m\right)}
$$

where $a_{i j}=a_{i}-a_{j}$. The proportionality factor $f(m)$ is independent of the internal parameter $a$. Since we will be concerned with difference operators acting only on the internal parameter $a$, we will not need to know the details of $f(m)$ and it will be omitted whenever convenient in what follows.

The complete correlation function on a torus with simple puncture is

$$
\int d a C(\alpha, 2 Q-\alpha, \mu) \overline{\mathcal{F}(\alpha, \mu ; \tau)} \mathcal{F}(\alpha, \mu ; \tau)
$$

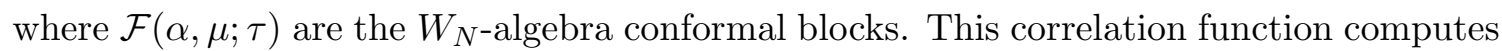
the ellipsoid partition function of the four-dimensional $\mathcal{N}=2^{*}$ theory on an ellipsoid, with the parameters identified as in the main text.

We now consider two different ways of factorizing the three-point function and absorbing it into the $W_{N}$-algebra conformal blocks. The first way is chosen to maximally simplify the expressions for the Verlinde operators and we expect that this corresponds to a half-sphere partition function of $\mathcal{N}=2^{*}$ theory with Dirichlet boundary conditions for the vectormultiplet. The second way corresponds to computing the Nekrasov partition function of the $\mathcal{N}=2^{*}$ theory with deformation parameters $\epsilon_{1}=b$ and $\epsilon_{2}=b^{-1}$.

\section{Renormalized conformal blocks}

Let us express the Toda three-point function in terms of double gamma functions and manipulate the answer into a convenient factorized form. For the hypermultiplet contribution, we have

$$
\begin{aligned}
\prod_{i, j=1}^{N} \Upsilon_{b}\left(\frac{q}{2}+i a_{i j}+i m\right)^{-1} & =\prod_{i, j=1}^{N} \Gamma_{b}\left(\frac{q}{2}+i a_{i j}+i m\right) \Gamma_{b}\left(\frac{q}{2}-i a_{i j}-i m\right) \\
& =\left|\prod_{i, j=1}^{N} \Gamma_{b}\left(\frac{q}{2}+i a_{i j}+i m\right)\right|^{2}
\end{aligned}
$$


For the vectormultiplet contribution

$$
\begin{aligned}
\prod_{i<j}^{N} \Upsilon_{b}\left(i a_{i j}\right) \Upsilon_{b}\left(-i a_{i j}\right) & =\prod_{i \neq j}^{N} \frac{1}{\Gamma_{b}\left(i a_{i j}\right) \Gamma_{b}\left(q-i a_{i j}\right)} \\
& =\prod_{i \neq j}^{N} \frac{\Gamma_{b}\left(q+i a_{i j}\right)}{\Gamma_{b}\left(i a_{i j}\right)} \prod_{i \neq j}^{N} \frac{1}{\Gamma_{b}\left(q+i a_{i j}\right) \Gamma_{b}\left(q-i a_{i j}\right)} \\
& =\prod_{i<j} \frac{S_{b}\left(q+i a_{i j}\right)}{S_{b}\left(i a_{i j}\right)}\left|\prod_{i \neq j}^{N} \frac{1}{\Gamma_{b}\left(q+i a_{i j}\right)}\right|^{2} \\
& =\mu(a)\left|\prod_{i<j}^{N} \frac{1}{\Gamma_{b}\left(q+i a_{i j}\right) \Gamma_{b}\left(q-i a_{i j}\right)}\right|^{2}
\end{aligned}
$$

where

$$
\mu(a)=\prod_{i<j} 2 \sinh \left(\pi b a_{i j}\right) 2 \sinh \left(\pi b^{-1} a_{i j}\right)
$$

is the $3 d$ partition function of an $\mathcal{N}=2$ vectormultiplet on a squashed three-sphere [54], which is identified here with the equator $\left\{x_{0}=0\right\}$.

As described in the main text, we can now absorb the three-point function into the $W_{N}$-algebra conformal blocks, by defining new renormalized blocks

$$
\mathcal{G}(a, m ; \tau)=\frac{\prod_{i, j=1}^{N} \Gamma_{b}\left(\frac{q}{2}+i a_{i j}+i m\right)}{\prod_{i<j}^{N} \Gamma_{b}\left(q+i a_{i j}\right) \Gamma_{b}\left(q-i a_{i j}\right)} \mathcal{F}(a, m ; \tau)
$$

such that the correlation function becomes

$$
\int d a \mu(a)|\mathcal{G}(a, m ; \tau)|^{2}
$$

We believe that the renormalized conformal block $\mathcal{G}(a, m ; \tau)$ correspond to the partition function on the upper half-sphere $\left\{x_{0}>0\right\}$ with Dirichlet boundary conditions for the vectormultiplet at the equator. Thus, in order to transform between Verlinde operators acting on $\mathcal{F}(a, m ; \tau)$ and those acting on $\mathcal{G}(a, m ; \tau)$ we have to conjugate by the factor in equation (C.8).

Let us concentrate on the Verlinde operator corresponding to the fundamental 't Hooft loop. Acting on the unnormalized conformal blocks, the difference operator has been computed in [30]. The result is given by

$$
\sum_{j=1}^{N}\left[\prod_{k \neq j}^{N} \frac{\Gamma\left(i b a_{k j}\right)}{\Gamma\left(\frac{b q}{2}+i b a_{k j}-i b m\right)} \frac{\Gamma\left(b q+i b a_{k j}\right)}{\Gamma\left(\frac{b q}{2}+i b a_{k j}+i b m\right)}\right] \Delta_{j}
$$

where we have introduced the notation $\Delta_{j}: a \rightarrow a+i b h_{j}$. Now, by patient and repeated application of the functional equation for the double gamma function, we find 


$$
\begin{aligned}
{\left[\frac{\prod_{i, j=1}^{N} \Gamma_{b}\left(\frac{q}{2}+i a_{i j}+i m\right)}{\prod_{i<j}^{N} \Gamma_{b}\left(q+i a_{i j}\right) \Gamma_{b}\left(q-i a_{i j}\right)}\right] \Delta_{j}\left[\frac{\prod_{i, j=1}^{N} \Gamma_{b}\left(\frac{q}{2}+i a_{i j}+i m\right)}{\prod_{i<j}^{N} \Gamma_{b}\left(q+i a_{i j}\right) \Gamma_{b}\left(q-i a_{i j}\right)}\right]^{-1} } \\
=\prod_{k \neq j}^{N} \frac{\Gamma\left(\frac{q b}{2}+i b a_{k j}+i b m\right)}{\Gamma\left(1-\frac{q b}{2}-i b a_{k j}+i b m\right)} \frac{\Gamma\left(1-i b a_{k j}\right)}{\Gamma\left(b q+i b a_{k j}\right)}
\end{aligned}
$$

We can immediately see that two sets of gamma functions the second line will cancel against the same gamma functions in the unnormalized operator in (C.10). The remaining gamma functions combine to give only trigonometric functions for the renormalized operator,

$$
\begin{array}{r}
\sum_{j=1}^{N}\left[\prod_{k \neq j}^{N} \frac{\Gamma\left(i b a_{k j}\right) \Gamma\left(1-i b a_{k j}\right)}{\Gamma\left(\frac{b q}{2}+i b a_{k j}-i b m\right) \Gamma\left(1-\frac{q b}{2}-i b a_{k j}+i b m\right)}\right] \Delta_{j} \\
=\sum_{j=1}^{N}\left[\prod_{k \neq j}^{N} \frac{\sin \pi b\left(\frac{q}{2}+i a_{k j}-i m\right)}{\sin \pi b\left(i a_{k j}\right)}\right] \Delta_{j}
\end{array}
$$

as claimed in the main text. With patient bookkeeping, the same computation can be performed for the difference operators in any other completely antisymmetric tensor representation.

\section{Nekrasov partition function}

For comparison with the exact computation of an 't Hooft loop on the four-sphere in [27], it is necessary to consider another factorization of the Toda three-point function. In this factorization the difference operators act on the Nekrasov partition function $\mathcal{Z}(a, m ; \tau)$, with $\epsilon_{1}=b$ and $\epsilon_{2}=b^{-1}$, which we named as in the main text.

Thus we now express the three-point function as

$$
C(\alpha, 2 Q-\alpha, \mu)=f(m)\left|\mathcal{Z}^{1-\operatorname{loop}}(a, m ; \tau)\right|^{2},
$$

where

$$
\mathcal{Z}^{1-\operatorname{loop}}(a, m ; \tau)=\left[\frac{\prod_{i<j}^{N} \Upsilon_{b}\left(i a_{i j}\right) \Upsilon_{b}\left(-i a_{i j}\right)}{\prod_{i, j=1}^{N} \Upsilon_{b}\left(\frac{q}{2}+i a_{i j}+i m\right)}\right]^{1 / 2}
$$

are the one-loop contributions to the Nekrasov partition function and $f(m)$ is independent of the internal momentum $a$ as before. The classical and instanton contributions to the Nekrasov partition function are encoded in the $W_{N}$-algebra conformal blocks. Thus, up to the factor $f(m)$, the complete Toda correlator can be expressed

$$
\int d a|\mathcal{Z}(a, m ; \tau)|^{2}
$$


in agreement with the exact computation of the partition function of the $\mathcal{N}=2^{*}$ theory on an ellipsoid in [39].

To obtain difference operators acting on the Nekrasov partition function, it is easier at this stage to start from the relationship to the renormalized $W_{N}$-algebra conformal blocks. In fact, from the relationship between the double gamma, the double sine functions and upsilon functions, we find that

$$
\mathcal{Z}(a, m ; \tau)=\left[\frac{\prod_{i<j}^{N} S_{b}\left(q+i a_{i j}\right) S_{b}\left(q-i a_{i j}\right)}{\prod_{i, j=1}^{N} S_{b}\left(\frac{q}{2}+i a_{i j}+i m\right)}\right]^{1 / 2} \mathcal{G}(a, m ; \tau) .
$$

Now, using the functional equation for the double sine function, we compute

$$
\begin{gathered}
{\left[\frac{\prod_{i<j}^{N} S_{b}\left(q+i a_{i j}\right) S_{b}\left(q-i a_{i j}\right)}{\prod_{i, j=1}^{N} S_{b}\left(\frac{q}{2}+i a_{i j}+i m\right)}\right]^{1 / 2} \Delta_{j}\left[\frac{\prod_{i<j}^{N} S_{b}\left(q+i a_{i j}\right) S_{b}\left(q-i a_{i j}\right)}{\prod_{i, j=1}^{N} S_{b}\left(\frac{q}{2}+i a_{i j}+i m\right)}\right]^{-1 / 2}} \\
=\left[\prod_{k \neq j}^{N} \frac{\sin \pi b\left(\frac{q}{2}+i a_{k j}+i m\right) \sin \pi b\left(i a_{k j}\right)}{\sin \pi b\left(\frac{q}{2}+i a_{k j}-i m\right) \sin \pi b\left(q+i a_{k j}\right)}\right]^{1 / 2} .
\end{gathered}
$$

Thus, combining with equation (C.12), we conjecture that the fundamental 't Hooft loop operator acting on the Nekrasov partition function with $\epsilon_{1}=b$ and $\epsilon_{2}=b^{-1}$ has the general form

$$
\sum_{j=1}^{k}\left[\prod_{k \neq j}^{N} \frac{\sin \pi b\left(\frac{q}{2}+i a_{k j}+i m\right) \sin \pi b\left(\frac{q}{2}+i a_{k j}-i m\right)}{\sin \pi b\left(i a_{k j}\right) \sin \pi b\left(q+i a_{k j}\right)}\right]^{1 / 2} \Delta_{j} .
$$

This agrees with the exact computation of the fundamental 't Hooft loop operator in the case of a round four-sphere $b=1$ [27]. Again, with patient bookkeeping the same conclusion can be reached for 't Hooft loops labeled by any antisymmetric tensor representation.

Open Access. This article is distributed under the terms of the Creative Commons Attribution License (CC-BY 4.0), which permits any use, distribution and reproduction in any medium, provided the original author(s) and source are credited.

\section{References}

[1] S. Gukov and E. Witten, Gauge theory, ramification, and the geometric Langlands program, hep-th/0612073 [INSPIRE].

[2] D. Gaiotto, $N=2$ dualities, JHEP 08 (2012) 034 [arXiv:0904.2715] [INSPIRE].

[3] D. Gaiotto, G.W. Moore and A. Neitzke, Wall-crossing, Hitchin systems and the WKB approximation, arXiv:0907.3987 [INSPIRE]. 
[4] L.F. Alday and Y. Tachikawa, Affine SL(2) conformal blocks from $4 d$ gauge theories, Lett. Math. Phys. 94 (2010) 87 [arXiv:1005.4469] [InSPIRE].

[5] H. Kanno and Y. Tachikawa, Instanton counting with a surface operator and the chain-saw quiver, JHEP 06 (2011) 119 [arXiv:1105.0357] [INSPIRE].

[6] G. Moore, Lecture notes for Felix Klein lectures, http://www.physics.rutgers.edu/ gmoore/FelixKleinLectureNotes.pdf.

[7] L.F. Alday, D. Gaiotto and Y. Tachikawa, Liouville correlation functions from four-dimensional gauge theories, Lett. Math. Phys. 91 (2010) 167 [arXiv:0906.3219] [INSPIRE].

[8] N. Wyllard, $A_{N-1}$ conformal Toda field theory correlation functions from conformal $N=2$ $\mathrm{SU}(N)$ quiver gauge theories, JHEP 11 (2009) 002 [arXiv:0907.2189] [INSPIRE].

[9] L.F. Alday, D. Gaiotto, S. Gukov, Y. Tachikawa and H. Verlinde, Loop and surface operators in $N=2$ gauge theory and Liouville modular geometry, JHEP 01 (2010) 113 [arXiv:0909.0945] [INSPIRE].

[10] D. Gaiotto, L. Rastelli and S.S. Razamat, Bootstrapping the superconformal index with surface defects, JHEP 01 (2013) 022 [arXiv:1207.3577] [INSPIRE].

[11] J. Kinney, J.M. Maldacena, S. Minwalla and S. Raju, An index for 4 dimensional super conformal theories, Commun. Math. Phys. 275 (2007) 209 [hep-th/0510251] [INSPIRE].

[12] A. Gadde, E. Pomoni, L. Rastelli and S.S. Razamat, S-duality and $2 d$ topological QFT, JHEP 03 (2010) 032 [arXiv:0910.2225] [INSPIRE].

[13] A. Gadde, L. Rastelli, S.S. Razamat and W. Yan, The superconformal index of the $E_{6} S C F T$, JHEP 08 (2010) 107 [arXiv: 1003.4244] [INSPIRE].

[14] A. Gadde, L. Rastelli, S.S. Razamat and W. Yan, The 4d superconformal index from q-deformed $2 d$ Yang-Mills, Phys. Rev. Lett. 106 (2011) 241602 [arXiv:1104.3850] [InSPIRE].

[15] A. Gadde, L. Rastelli, S.S. Razamat and W. Yan, Gauge theories and Macdonald polynomials, Commun. Math. Phys. 319 (2013) 147 [arXiv:1110.3740] [InSPIRE].

[16] D. Gaiotto and S.S. Razamat, Exceptional indices, JHEP 05 (2012) 145 [arXiv:1203.5517] [INSPIRE].

[17] L.F. Alday, M. Bullimore, M. Fluder and L. Hollands, Surface defects, the superconformal index and q-deformed Yang-Mills, JHEP 10 (2013) 018 [arXiv:1303.4460] [INSPIRE].

[18] T. Dimofte, S. Gukov and L. Hollands, Vortex counting and Lagrangian 3-manifolds, Lett. Math. Phys. 98 (2011) 225 [arXiv: 1006. 0977] [INSPIRE].

[19] D. Gaiotto, Surface operators in $N=2$ 4d gauge theories, JHEP 11 (2012) 090 [arXiv: 0911.1316] [INSPIRE].

[20] D. Gaiotto, S. Gukov and N. Seiberg, Surface defects and resolvents, JHEP 09 (2013) 070 [arXiv: 1307.2578] [INSPIRE].

[21] A. Gadde and S. Gukov, 2d index and surface operators, JHEP 03 (2014) 080 [arXiv: 1305.0266] [INSPIRE].

[22] M. Aganagic and S. Shakirov, Knot homology from refined Chern-Simons theory, arXiv: 1105.5117 [INSPIRE]. 
[23] A. Gadde and W. Yan, Reducing the $4 d$ index to the $S^{3}$ partition function, JHEP 12 (2012) 003 [arXiv: 1104.2592] [INSPIRE].

[24] F.A.H. Dolan, V.P. Spiridonov and G.S. Vartanov, From 4d superconformal indices to $3 d$ partition functions, Phys. Lett. B 704 (2011) 234 [arXiv:1104.1787] [INSPIRE].

[25] Y. Imamura, Relation between the $4 d$ superconformal index and the $S^{3}$ partition function, JHEP 09 (2011) 133 [arXiv: 1104.4482] [INSPIRE].

[26] F. Benini, Y. Tachikawa and D. Xie, Mirrors of 3d Sicilian theories, JHEP 09 (2010) 063 [arXiv: 1007.0992] [INSPIRE].

[27] J. Gomis, T. Okuda and V. Pestun, Exact results for 't Hooft loops in gauge theories on $S^{4}$, JHEP 05 (2012) 141 [arXiv:1105.2568] [INSPIRE].

[28] N. Drukker, J. Gomis, T. Okuda and J. Teschner, Gauge theory loop operators and Liouville theory, JHEP 02 (2010) 057 [arXiv: 0909.1105] [INSPIRE].

[29] N. Drukker, D. Gaiotto and J. Gomis, The virtue of defects in $4 d$ gauge theories and $2 d$ CFTs, JHEP 06 (2011) 025 [arXiv:1003.1112] [INSPIRE].

[30] J. Gomis and B. Le Floch, 't Hooft operators in gauge theory from Toda CFT, JHEP 11 (2011) 114 [arXiv:1008.4139] [INSPIRE].

[31] N. Doroud, J. Gomis, B. Le Floch and S. Lee, Exact results in $D=2$ supersymmetric gauge theories, JHEP 05 (2013) 093 [arXiv: 1206.2606] [INSPIRE].

[32] F. Benini and S. Cremonesi, Partition functions of $N=(2,2)$ gauge theories on $S^{2}$ and vortices, arXiv:1206.2356 [INSPIRE].

[33] D. Gaiotto and E. Witten, Supersymmetric boundary conditions in $N=4$ super Yang-Mills theory, J. Statist. Phys. 135 (2009) 789 [arXiv:0804.2902] [INSPIRE].

[34] D. Gaiotto and E. Witten, S-duality of boundary conditions in $N=4$ super Yang-Mills theory, Adv. Theor. Math. Phys. 13 (2009) 721 [arXiv:0807.3720] [INSPIRE].

[35] K. Hosomichi, S. Lee and J. Park, AGT on the S-duality wall, JHEP 12 (2010) 079 [arXiv: 1009.0340] [INSPIRE].

[36] S. Benvenuti and S. Pasquetti, 3d-partition functions on the sphere: exact evaluation and mirror symmetry, JHEP 05 (2012) 099 [arXiv: 1105.2551] [INSPIRE].

[37] T. Nishioka, Y. Tachikawa and M. Yamazaki, 3d partition function as overlap of wavefunctions, JHEP 08 (2011) 003 [arXiv: 1105.4390] [INSPIRE].

[38] Y. Terashima and M. Yamazaki, SL $(2, R)$ Chern-Simons, Liouville and gauge theory on duality walls, JHEP 08 (2011) 135 [arXiv:1103.5748] [INSPIRE].

[39] N. Hama and K. Hosomichi, Seiberg-Witten theories on ellipsoids, JHEP 09 (2012) 033 [Addendum ibid. 10 (2012) 051] [arXiv: 1206 . 6359] [INSPIRE].

[40] V. Pestun, Localization of gauge theory on a four-sphere and supersymmetric Wilson loops, Commun. Math. Phys. 313 (2012) 71 [arXiv:0712.2824] [INSPIRE].

[41] N.A. Nekrasov, Seiberg-Witten prepotential from instanton counting, Adv. Theor. Math. Phys. 7 (2004) 831 [hep-th/0206161] [INSPIRE].

[42] N. Nekrasov and A. Okounkov, Seiberg-Witten theory and random partitions, hep-th/0306238 [INSPIRE]. 
[43] J. Teschner, From Liouville theory to the quantum geometry of Riemann surfaces, hep-th/0308031 [INSPIRE].

[44] A. Kapustin, Wilson-'t Hooft operators in four-dimensional gauge theories and S-duality, Phys. Rev. D 74 (2006) 025005 [hep-th/0501015] [InSPIRE].

[45] V.A. Fateev and A.V. Litvinov, On differential equation on four-point correlation function in the conformal Toda field theory, JETP Lett. 81 (2005) 594 [hep-th/0505120] [INSPIRE].

[46] V.A. Fateev and A.V. Litvinov, Correlation functions in conformal Toda field theory. I, JHEP 11 (2007) 002 [arXiv:0709.3806] [InSPIRE].

[47] G. Felder and A. Varchenko, Elliptic quantum groups and Ruijsenaars models, J. Statist. Phys. 89 (1996) 963 [q-alg/9704005].

[48] A. Kapustin, B. Willett and I. Yaakov, Exact results for supersymmetric Abelian vortex loops in $2+1$ dimensions, JHEP 06 (2013) 099 [arXiv:1211.2861] [INSPIRE].

[49] N. Drukker, T. Okuda and F. Passerini, Exact results for vortex loop operators in $3 d$ supersymmetric theories, JHEP 07 (2014) 137 [arXiv:1211.3409] [INSPIRE].

[50] D. Gaiotto and E. Witten, Janus configurations, Chern-Simons couplings, and the $\theta$-angle in $N=4$ super Yang-Mills theory, JHEP 06 (2010) 097 [arXiv:0804.2907] [INSPIRE].

[51] L. Hadasz, Z. Jaskolski and P. Suchanek, Modular bootstrap in Liouville field theory, Phys. Lett. B 685 (2010) 79 [arXiv:0911.4296] [INSPIRE].

[52] T. Okuda and V. Pestun, On the instantons and the hypermultiplet mass of $N=2 *$ super Yang-Mills on $S^{4}$, JHEP 03 (2012) 017 [arXiv: 1004.1222] [INSPIRE].

[53] G. Vartanov and J. Teschner, Supersymmetric gauge theories, quantization of moduli spaces of flat connections and conformal field theory, arXiv:1302.3778 [INSPIRE].

[54] N. Hama, K. Hosomichi and S. Lee, SUSY gauge theories on squashed three-spheres, JHEP 05 (2011) 014 [arXiv:1102.4716] [INSPIRE]. 